\title{
Double Hopf bifurcation in delayed reaction-diffusion systems
}

\author{
Yanfei Du \\ Shaanxi University of Science and Technology, Xi'an 710021, China. \\ Ben Niu*, Yuxiao Guo, Junjie Wei \\ Department of Mathematics, Harbin Institute of Technology, Weihai 264209, China. \\ *Corresponding author, niu@hit.edu.cn
}

(Dated: November 27, 2018)

\begin{abstract}
Double Hopf bifurcation analysis can be used to reveal some complicated dynamical behavior in a dynamical system, such as the existence or coexistence of periodic orbits, quasi-periodic orbits, or even chaos. In this paper, an algorithm for deriving the normal form near a codimension-two double Hopf bifurcation of a reaction-diffusion system with time delay and Neumann boundary condition is rigorously established, by employing the center manifold reduction technique and the normal form method. We find that the dynamical behavior near bifurcation points are proved to be governed by twelve distinct unfolding systems. Two examples are performed to illustrate our results: for a stage-structured epidemic model, we find that double Hopf bifurcation appears when varying the diffusion rate and time delay, and two stable spatially inhomogeneous periodic oscillations are proved to coexist near the bifurcation point; in a diffusive predator-prey system, we theoretically proved that quasi-periodic orbits exist on two- or three-torus near a double Hopf bifurcation point, which will break down after slight perturbation, leaving the system a strange attractor.
\end{abstract}

Keywords: Reaction-diffusion model, delay, double Hopf bifurcation, coexistence, strange attractor 


\section{INTRODUCTION}

A Hopf bifurcation refers to the phenomenon that steady states lose their stability and give rise to periodic solutions, as a parameter crosses critical values [2, 28, 32, 42, 52]. Since the condition of existence of Hopf bifurcation can be easily verified, and both the direction of Hopf bifurcation and the stability of the bifurcating periodic solution can be determined by formulas derived from center manifold reduction technique, Hopf bifurcation analysis has been an effective method of the study on the existence of periodic solutions of differential equations. In recent years, Hopf bifurcation has been intensely studied to investigate the dynamics of differential equations in various fields [6, 31, 43, 48]. Normal forms theory provides powerful tools to bifurcation analysis, whose basic idea is to employ near-identity nonlinear transformations that lead the original system to a qualitatively equivalent differential equation with the simplest form.

In a reaction-diffusion system, especially reaction-diffusion system with delays, there may exist several Hopf bifurcation critical points with parameters' varying. Thus, like the case in ordinary differential equations [36], double Hopf bifurcations will be characterized by intersections of two Hopf bifurcation curves in a two-parameter plane. In fact, a double Hopf bifurcation occurs from a critical point at which the Jacobian evaluated there involves two conjugate pairs of pure imaginary eigenvalues. Guckenheimer and Holmes [25] gave the dynamics in the neighborhood of a codimension-two double Hopf point in an ordinary differential equation. When bifurcation parameters are closed to the double Hopf bifurcation point, the system may exhibit rich dynamics, such as periodic oscillations, quasi-periodic oscillations, coexisting of several oscillations, two- or three-dimensional invariant torus, and even chaos [25, 36, 60]. For delayed systems governed by delay differential equations, due to the fact that delay differential equations are of infinite dimensional, the center manifold method was adopted to reduce delay differential equations into finite-dimensional systems, and then normal forms can be calculated on the center manifold [8, 9, 17, 21, 22, 29]. Correspondingly, the multiple timescale method has also been successfully applied to analyze codimension-two non-resonant double Hopf bifurcations [39, 59]. Research on the double Hopf bifurcation and complex dynamics of delay differential equations is also carried out by these methods we mentioned above [7, 10, 40, 61, 62].

In recent years, the analysis of Hopf bifurcation in a reaction-diffusion systems has at- 
tracted much attention. Based on the method provided in [30, 53], Yi et al. [58] carried out Hopf bifurcation analysis in a reaction-diffusion system without delays. By decomposing the equation with the orthonormal Fourier basis corresponding to the eigenvalue problem, an explicit expression is given for several key parameters in determining the properties of bifurcation according to the method given in [30]. Faria [19] investigated the effect of both time delay and diffusion on Hopf bifurcation, and improved the method given in [20, 21] to study normal forms with perturbation parameters of Hopf bifurcation in delayed reaction-diffusion equations with Neumann boundary condition. The results in [19] have been further applied to equations with various practical backgrounds, such as the predator-prey model [12] and so on. Recently, Hopf bifurcation analysis of delayed reaction-diffusion equations with Dirichlet boundary condition has also made considerable progress, such as in [27, 51, 57], the existence of Hopf bifurcation and the stability and direction of bifurcating periodic solutions for some population models with Dirichlet boundary condition were considered; Furthermore, Guo [26], Chen and Yu [13], combining the non-local phenomenon, considered Hopf bifurcation of the delayed reaction-diffusion equations with Dirichlet boundary condition. Comparing the research on Dirichlet boundary conditions with the research on Neumann boundary conditions, the main difficulty when investigating such models is the existence of positive steady-state solutions and the complexity of the corresponding eigenvalue problem, so practical analysis is usually carried out by combining the method of phase space analysis, topological degree theory or Liapunov-Schmidt reduction theory [35]. In this paper, only homogeneous Neumann boundary condition is considered, as we can assume that zero is an equilibrium of the system.

For analysis on high codimensional bifurcations of reaction-diffusion equations, so far as we know, there are very few published results. Only a few results have been conducted mainly on the research of Turing-Hopf bifurcation. Research on interaction between the Turing bifurcation and Hopf bifurcation in reaction-diffusion equations can be traced back to the work of De Wit et al. [14]. Later, Meixner et al. [41] conducted a preliminary analysis of the problem through codimension-two bifurcation analysis. Parallel research was also seen in literature [5] on the predator-prey system. Song et al. [49] investigated the Turing-Hopf bifurcation from the point of view of analysis of bifurcation and normal forms, in which they extended the method given in [19, 58], and deduced the normal form with parameters of Turing-Hopf bifurcation. Xu and Wei [56] applied this method to study the 
Turing-Hopf bifurcation of a predator-prey model. Turing-Hopf bifurcation can be seen as a special case of Hopf-zero bifurcation of dynamic system from the point of view of classification of bifurcation. Results about Hopf-zero bifurcations and normal form analysis in delayed reaction-diffusion equations can be found in An and Jiang's preprint [1]. For double Hopf bifurcation, Lewis and Nagata [37] studied the transitions from axisymmetric steady solutions to nonaxisymmetric waves in a Navier-Stokes model of the differentially heated rotating annulus experiment, and an analytical-numerical center manifold reduction is used to analyze the double Hopf bifurcation points that occur at this transition. Besides [37], to our best knowledge, there have not been any results on double Hopf bifurcation analysis and the corresponding normal form calculation in the reaction-diffusion equations with or without delays.

Based on the general method of normal form simplification in ordinary differential equation or delay differential equation, the ratio of two pairs of imaginary roots appearing at the double Hopf bifurcation point is important to determine the final simplest normal form. When the ratio is not a rational number, the normal form has a universal form as those provided in [7, 8, 10, 39, 40, 59, 61, 62]. However, if the ratio is rational, there must exist some additional terms which couldn't be eliminated because of the resonance. Precisely, if we are about to obtain the topological classification of the dynamical behavior near the double Hopf points by analyzing the third order normal form, this only require that the ratio is not $m: n$ for $m, n=1,2,3,4$, namely, weak resonance case. For all weakly resonant double Hopf bifurcation, we can treat them in the same method as that used for non-resonant cases. Otherwise, if the ratio is $m: n$ for $m, n=1,2,3,4$, we say that this is a strongly resonance case, which has also been investigated in ordinary differential equations and delay differential equations [3, 11, 23, 24, 34, 44, 45, 50].

In this paper, we aim at the development of a method of computing normal forms on center manifold near an equilibrium of a system of a general delayed reaction-diffusion equations with non-resonant or weakly resonant double Hopf singularity. Consider a general reaction-diffusion model

$$
\left\{\begin{array}{l}
\frac{\partial u_{1}(x, t)}{\partial t}=d_{1}(\mu) \Delta u_{1}(x, t)+f_{1}\left(\mu, u_{1}^{t}, u_{2}^{t}, \cdots, u_{n}^{t}\right), \\
\frac{\partial u_{2}(x, t)}{\partial t}=d_{2}(\mu) \Delta u_{2}(x, t)+f_{2}\left(\mu, u_{1}^{t}, u_{2}^{t}, \cdots, u_{n}^{t}\right), \\
\vdots \\
\frac{\partial u_{n}(x, t)}{\partial t}=d_{n}(\mu) \Delta u_{n}(x, t)+f_{n}\left(\mu, u_{1}^{t}, u_{2}^{t}, \cdots, u_{n}^{t}\right),
\end{array} \quad x \in(0, l \pi),\right.
$$


with homogeneous Neumann boundary conditions and $u_{i}^{t}(\theta)(x)=u_{i}(t+\theta, x)$. Define $f_{i}(\mu, 0, \cdots, 0)=0, i=1,2, \cdots, n$, such that (1) has zero equilibrium, and assume that every $f_{i}$ is at least $C^{3}$.

We will extend the normal form method given by Faria and Magalhães [18, 21] to investigate non-resonant or weakly resonant double Hopf bifurcations in reaction-diffusion systems with delays. The first step of this method is to decompose the phase space, and then to decompose the equations we investigate. Then we can calculate the second and third order normal form on the center manifold, and get the normal form for double Hopf bifurcation, which is a four-dimensional system up to the third order with unfolding parameters restricted to the center manifold. By polar coordinates transformation, the four-dimensional system can be reduced to a two-dimensional amplitude system, in which the unfolding parameters can be expressed by the parameters in the original system. Due to the unfolding analysis given in [25], we can determine the type of twelve distinct kinds of unfoldings by the coefficients we calculated in the normal form, and get corresponding bifurcation sets. By detailed analysis of bifurcation sets, all the dynamical behaviors near the double Hopf bifurcation point can be figured out. In this paper, a series of explicit calculation formulas of the normal form for non-resonant or weakly resonant double Hopf bifurcation is given, with three different cases of wave number: $n_{1}=0, n_{2}=0, n_{1}=0, n_{2} \neq 0$, and $n_{1} \neq 0, n_{2} \neq 0$. The wave number is related to the spatial profile of bifurcating periodic solutions.

To illustrate the calculation process of the normal forms, we perform two examples. The first one is a diffusive, stage-structured epidemic model with two delays. Xiao and Chen [55] proposed an SIS epidemic model with stage structure and time delays in the following form

$$
\left\{\begin{array}{l}
\frac{d S}{d t}=\alpha y(t)-d S(t)-\alpha e^{-d \tau} y(t-\tau)-\mu S(t-\omega) I(t)+\gamma I(t) \\
\frac{d I}{d t}=\mu S(t-\omega) I(t)-d I(t)-\gamma I(t) \\
\frac{d y}{d t}=\alpha e^{-d \tau} y(t-\tau)-\beta y^{2}(t)
\end{array}\right.
$$

where $S(t)$ and $I(t)$ are the population of susceptible and infected immature individuals, $y(t)$ represents the population of mature individuals, $\tau$ is the maturity delay, and $\omega$ is the freely moving delay. Hopf bifurcation results for such a model are given in Du et al. [15]. Adding random diffusion of individuals into (2), we have a diffusive epidemic model, which is proposed in section 4. We take the time delay $\omega$ and a diffusion coefficient as bifurcation parameters. We show that double Hopf bifurcation can appear with two different cases of 
wave numbers: $n_{1}=0, n_{2} \neq 0$, or $n_{1} \neq 0, n_{2} \neq 0$. Thus, the spatio-temporal dynamics turn out to be very complicated near the double Hopf bifurcation point. In some regions, there are two stable and spatially nonhomogeneous periodic solutions or a homogeneous and a nonhomogeneous periodic solution coexisting.

The second example we consider is a predator-prey system with time delay. Predatorprey systems have been widely investigated [33, 46, 54]. Here, we choose a simple system which was studied in [48] to illustrate the procedure of normal form calculation

$$
\begin{aligned}
& \frac{\mathrm{d} u(t)}{\mathrm{d} t}=u(t)\left[r_{1}-a_{11} u(t-\tau)-a_{12} v(t)\right], \\
& \frac{\mathrm{d} v(t)}{\mathrm{d} t}=v(t)\left[-r_{2}+a_{21} u(t)-a_{22} v(t)\right] .
\end{aligned}
$$

Incorporating diffusion terms into (3) , the system becomes a reaction-diffusion system, which is proposed in section 5. Taking $r_{1}$ and $\tau$ as bifurcation parameters, we will show that such a simple system will exhibit complicated dynamical behavior near the double Hopf bifurcation point such as the existence of quasi-periodic solution on a 2-torus and quasi-periodic solution on a 3-torus. Generally, a vanishing 3-torus might accompany strange attractors, and lead a system into chaos, which is called the "Ruelle-Takens-Newhouse" scenario [4, 16, 47]. Thus a strange attractor is found near the double Hopf bifurcation point in this predator-prey system.

The paper is organized as follows. In section 2, we discuss eigenfunctions and decomposition of the phase space. Section 3 is devoted to calculating the normal forms of non-resonant or weakly resonant double Hopf bifurcation of (1), and explicit formula of normal form truncated to the third order is obtained in three cases of different wave numbers. As applications of the method, a diffusive epidemic model with two delays and a diffusive predator-prey system with a delay are considered in sections 4 and 5 , respectively, where the conditions for the existence of double Hopf bifurcation are obtained, the normal forms are calculated using the method and formulas in section 3, Two-parameter unfoldings and bifurcation diagrams near the critical point are analyzed, and some numerical simulations about the rich dynamics near these bifurcation points are demonstrated. Finally, we close the paper with some conclusions. 


\section{EIGENFUNCTIONS AND DECOMPOSITION OF THE PHASE SPACE}

Normal form analysis usually depends on a decomposition of the corresponding phase space [36, 52]. In case of reaction-diffusion model with time delay, it also requires the decomposition with respect to spatial variables which can be accomplished by finding the eigenfunctions of Laplacian operator. Thus, we first rewrite system (11) into an abstract ordinary differential equation in an appropriate phase space.

\subsection{Eigenfunctions}

Using the general approach to put a partial differential equation into an abstract form introduced in [53], we define the real-valued Hilbert space

$$
X:=\left\{\left(u_{1}, u_{2}, \cdots, u_{n}\right)^{T} \in\left(H^{2}(0, l \pi)\right)^{n}: \frac{\partial u_{i}}{\partial x}(0, t)=\frac{\partial u_{i}}{\partial x}(l \pi, t)=0, i=1,2, \cdots, n\right\} .
$$

Since we are about to deal with the double Hopf bifurcations with two pairs of purely imaginary eigenvalues, we usually extend the space $X$ into the corresponding complexification space of $X$ in a natural way by

$$
X_{\mathbb{C}}:=X \oplus i X=\left\{U_{1}+i U_{2}: U_{1}, U_{2} \in X\right\}
$$

with the general complex-value $L^{2}$ inner product

$$
\langle u, v\rangle=\int_{0}^{l \pi}\left(\bar{u}_{1} v_{1}+\bar{u}_{2} v_{2}+\cdots+\bar{u}_{n} v_{n}\right) d x
$$

for $u=\left(u_{1}, u_{2}, \cdots, u_{n}\right)^{T}, v=\left(v_{1}, v_{2}, \cdots, v_{n}\right)^{T} \in X_{\mathbb{C}}$. Let $\mathcal{C}:=C\left([-r, 0], X_{\mathbb{C}}\right)$ denote the phase space with the sup norm. We write $u^{t} \in \mathcal{C}$ for $u^{t}(\theta)=u(t+\theta),-r \leq \theta \leq 0$.

Now we can rewrite system (11) into an abstract form as

$$
\begin{gathered}
\frac{\mathrm{d} U(t)}{\mathrm{d} t}=D(\mu) \Delta U(t)+L(\mu)\left(U^{t}\right)+F\left(\mu, U^{t}\right), \quad t>0, \\
\text { where } U(t)=\left(\begin{array}{c}
u_{1}(t) \\
u_{2}(t) \\
\vdots \\
u_{n}(t)
\end{array}\right) \in X_{\mathbb{C}}, u^{t}=\left(\begin{array}{c}
u_{1}^{t} \\
u_{2}^{t} \\
\vdots \\
u_{n}^{t}
\end{array}\right) \in \mathcal{C}, D(\mu)=\left(\begin{array}{cccc}
d_{1}(\mu) & 0 & \cdots & 0 \\
0 & d_{2}(\mu) & \cdots & 0 \\
\vdots & \vdots & \ddots & \vdots \\
0 & 0 & \cdots & d_{n}(\mu)
\end{array}\right) \text {, }
\end{gathered}
$$


$F\left(\mu, U^{t}\right)=\left(\begin{array}{c}F^{(1)}\left(\mu, U^{t}\right) \\ F^{(2)}\left(\mu, U^{t}\right) \\ \vdots \\ F^{(n)}\left(\mu, U^{t}\right)\end{array}\right)$, and $d_{i}(\mu)>0$ for $i=1,2, \cdots, n, \mu \in \mathbb{R}^{2} . L: \mathbb{R}^{2} \times \mathcal{C} \rightarrow X_{\mathbb{C}}$ is a bounded linear operator. $F(\mu, \phi) \in X_{\mathbb{C}}$ for $(\mu, \phi) \in \mathbb{R}^{2} \times \mathcal{C}$, and $F$ is a $C^{k}(k \geq 3)$ function such that $F(\mu, 0)=0, D_{\phi} F(\mu, 0)=0$, where $D_{\phi} F(\mu, 0)$ stands for the Fréchet derivative of $F(\mu, \phi)$ with respect to $\phi$ at $\phi=0$.

Linearizing system (4) at $(0,0, \cdots, 0)$, we have

$$
\frac{\mathrm{d} U(t)}{\mathrm{d} t}=D(\mu) \Delta U(t)+L(\mu)\left(U^{t}\right)
$$

It is well known that the eigenvalue problem

$$
\Delta \varphi=\lambda \varphi, \quad x \in(0, l \pi),\left.\varphi_{x}\right|_{x=0, l \pi}=0
$$

has eigenvalues

$$
\lambda_{m}=-\frac{m^{2}}{l^{2}}, m \in \mathbb{N}_{0}=\mathbb{N} \cup\{0\},
$$

with corresponding normalized eigenfunctions

$$
\gamma_{m}(x)=\frac{\cos \frac{m}{l} x}{\left\|\cos \frac{m}{l} x\right\|_{L^{2}}}= \begin{cases}\sqrt{\frac{1}{l \pi}}, & m=0 \\ \sqrt{\frac{2}{l \pi}} \cos \frac{m}{l} x, & m \geq 1\end{cases}
$$

Let $\beta_{m}^{(j)}(x)=\gamma_{m}(x) e_{j}$, where $e_{j}$ is the $j$-th unit coordinate vector of $\mathbb{R}^{n}$. Then $\left\{\beta_{m}^{(j)}\right\}_{m \geq 0}$ are eigenfunctions of $D(\mu) \Delta$ with corresponding eigenvalues $-d_{i}(\mu) \frac{m^{2}}{l^{2}}(i=1,2, \cdots n)$. Applying the general theory about elliptic operators, we know $\left\{\beta_{m}^{(j)}\right\}_{m \geq 0}$ form an orthnormal basis of $X$.

Define $\mathcal{B}_{m}$ the subspace of $\mathcal{C}$ by

$$
\mathcal{B}_{m}:=\operatorname{span}\left\{\left\langle v(\cdot), \beta_{m}^{(j)}\right\rangle \beta_{m}^{(j)} \mid v \in \mathcal{C}, j=1,2, \cdots, n\right\}
$$

For simplification of notations, in the following we write

$$
\left\langle v(\cdot), \beta_{m}\right\rangle=\left(\begin{array}{c}
\left\langle v(\cdot), \beta_{m}^{(1)}\right\rangle \\
\left\langle v(\cdot), \beta_{m}^{(2)}\right\rangle \\
\vdots \\
\left\langle v(\cdot), \beta_{m}^{(n)}\right\rangle
\end{array}\right) .
$$


The characteristic equation associated with (5) is

$$
\lambda y-D(\mu) \Delta y-L(\mu)\left(e^{\lambda \cdot} y\right)=0,
$$

where $e^{\lambda \cdot}(\theta) y=e^{\lambda \theta} y$, for $\theta \in[-r, 0]$. By using the Fourier expansion

$$
y=\sum_{m=0}^{\infty}\left(\begin{array}{c}
a_{1 m} \\
a_{2 m} \\
\vdots \\
a_{n m}
\end{array}\right) \gamma_{m}
$$

we find that the characteristic equation (6) is equivalent to a sequence of characteristic equations

$$
\operatorname{det}\left[\lambda I+\frac{m^{2}}{l^{2}} D(\mu)-L(\mu)\left(e^{\lambda \cdot} I\right)\right]=0, m \in \mathbb{N}_{0}
$$

In order to consider double Hopf bifurcation, we assume that the following conditions hold for some $\mu_{0} \in \mathbb{R}^{2}[36]$ :

$\left(H_{1}\right)$ There exists a neighborhood $O$ of $\mu_{0}$ such that for $\mu \in O$, (5) has two pairs of complex simple conjugate eigenvalues $\alpha_{1}(\mu) \pm i \omega_{1}(\mu)$ and $\alpha_{2}(\mu) \pm i \omega_{2}(\mu)$, all continuously differential in $\mu$ with $\alpha_{1}\left(\mu_{0}\right)=0, \omega_{1}\left(\mu_{0}\right)=\omega_{1}>0, \alpha_{2}\left(\mu_{0}\right)=0, \omega_{2}\left(\mu_{0}\right)=\omega_{2}>0$, and the remaining eigenvalues of (5) have non-zero real part for $\mu \in O$.

$\left(H_{2}\right)$ Assume that $\omega_{1}<\omega_{2}$ and $\omega_{1}: \omega_{2} \neq i: j$ for $i, j \in \mathbb{N}$ and $1 \leq i \leq j \leq 4$, i.e., we do not consider the strongly resonant cases.

$\left(H_{3}\right)$ The conjugate eigenvalues $\alpha_{k}(\mu) \pm i \omega_{k}(\mu)$ are obtained by $\left(7_{h_{k}}\right)$ and the corresponding eigenvalues belong to $\mathcal{B}_{n_{k}}, k=1,2$, and $n_{1}, n_{2} \in \mathbb{N}_{0}$. Assume that $n_{1} \leq n_{2}$.

To figure out the spatiotemporal dynamical behavior near the critical point $\mu=\mu_{0}$, let $\mu=\alpha+\mu_{0}$, where $\alpha \in \mathbb{R}^{2}$. Now the system (5) is equivalent to

$$
\frac{\mathrm{d} U(t)}{\mathrm{dt}}=D_{0} \Delta U(t)+L_{0}\left(U^{t}\right)+\widetilde{F}\left(\alpha, U^{t}\right)
$$

where $D_{0}=D\left(\mu_{0}\right), L_{0}(\cdot)=L\left(\mu_{0}\right)(\cdot)$ is a linear operator from $\mathcal{C}$ to $X_{\mathbb{C}}$, and $\widetilde{F}(\alpha, \varphi)=$ $\left[D\left(\alpha+\mu_{0}\right)-D_{0}\right] \Delta \varphi(0)+\left[L\left(\alpha+\mu_{0}\right)-L_{0}\right](\varphi)+F\left(\alpha+\mu_{0}, \varphi\right)$. 


\subsection{Decomposition of the Phase Space}

In order to adapt the center manifold reduction technique, we have to operate on an equation about $U^{t}$, which requires an enlarged phase space $\mathcal{B C}$ defined by

$$
\mathcal{B C}:=\left\{\psi:[-r, 0] \rightarrow X_{\mathbb{C}}: \psi \text { is continuous on }[-r, 0), \exists \lim _{\theta \rightarrow 0^{-}} \psi(\theta) \in X_{\mathbb{C}}\right\} .
$$

Eq. (8) can be rewritten as an abstract ordinary differential equation on $\mathcal{B C}$ [18]:

$$
\frac{\mathrm{d} U^{t}}{\mathrm{~d} t}=A U^{t}+X_{0} \widetilde{F}\left(\alpha, U^{t}\right)
$$

where $A$ is the infinitesimal generator of the $C_{0}$-semigroup of solution maps of the linear equation (5), defined by

$$
A: \mathcal{C}_{0}^{1} \cap \mathcal{B C} \rightarrow \mathcal{B C}, A \varphi=\dot{\varphi}+X_{0}\left[D_{0} \Delta \varphi(0)+L_{0}(\varphi)-\dot{\varphi}(0)\right]
$$

with $\operatorname{dom}(A)=\{\varphi \in \mathcal{C}: \dot{\varphi} \in \mathcal{C}, \varphi(0) \in \operatorname{dom}(\Delta)\}$ and $X_{0}$ is given by

$$
X_{0}(\theta)=\left\{\begin{array}{l}
0, \quad-r \leq \theta<0 \\
I, \quad \theta=0
\end{array}\right.
$$

Then on $\mathcal{B}_{m}$, the linear equation

$$
\frac{\mathrm{d} U(t)}{\mathrm{d} t}=D_{0} \Delta U(t)+L_{0}\left(U^{t}\right)
$$

is equivalent to the retarded functional differential equation on $\mathbb{C}^{n}$ :

$$
\dot{y}(t)=-\frac{m^{2}}{l^{2}} D_{0} y(t)+L_{0} y^{t} .
$$

Define functions of bounded variation $\eta_{k} \in B C 0([-r, 0], \mathbb{R})$ for $n_{k}(k=1,2)$ mentioned in $\left(H_{3}\right)$, such that

$$
-\frac{n_{k}^{2}}{l^{2}} D_{0} \varphi(0)+L_{0}(\varphi)=\int_{-r}^{0} d \eta_{k}(\theta) \varphi(\theta), \varphi \in \mathcal{C}
$$

Let $A_{k}(k=1,2)$ denote the infinitesimal generator of the semigroup defined by (11) with $\mu=\mu_{0}, m=n_{1}, n_{2}$, and $A_{k}^{*}$ denotes the formal adjoint of $A_{k}$ under the bilinear form

$$
(\psi, \phi)_{k}=\psi(0) \phi(0)-\int_{-r}^{0} \int_{0}^{\theta} \psi(\xi-\theta) d \eta_{k}(\theta) \phi(\xi) d \xi
$$

Let

$$
\begin{aligned}
& \Phi_{1}(\theta)=\left(\phi_{1}(\theta), \phi_{2}(\theta)\right), \quad \Phi_{2}(\theta)=\left(\phi_{3}(\theta), \phi_{4}(\theta)\right), \\
& \Psi_{1}(s)=\left(\begin{array}{l}
\psi_{1}(s) \\
\psi_{2}(s)
\end{array}\right), \quad \Psi_{2}(s)=\left(\begin{array}{l}
\psi_{3}(s) \\
\psi_{4}(s)
\end{array}\right),
\end{aligned}
$$


be the basis of the generalized eigenspace of $A_{k}, A_{k}^{*}$ corresponding to the eigenvalues $i \omega_{1}$ and $i \omega_{2}$, respectively, and satisfying

$$
A_{k} \Phi_{k}=\Phi_{k} B_{k}, A_{k}^{*} \Psi_{k}=B_{k} \Psi_{k},\left(\Psi_{k}, \Phi_{k}\right)_{k}=I, k=1,2
$$

with $B_{1}=\operatorname{diag}\left(i \omega_{1},-i \omega_{1}\right), B_{2}=\operatorname{diag}\left(i \omega_{2},-i \omega_{2}\right)$. Denote $\Phi(\theta)=\left(\Phi_{1}(\theta), \Phi_{2}(\theta)\right)$, and $\Psi(s)=$ $\left(\Psi_{1}(s), \Psi_{2}(s)\right)^{T}$. Now, we can decompose $\mathcal{B C}$ into a center subspace and its orthocomplement, i.e.,

$$
\mathcal{B C}=\mathcal{P} \oplus \operatorname{Ker} \pi
$$

where $\pi: \mathcal{B C} \rightarrow \mathcal{P}$ is the projection defined by

$$
\pi(\varphi)=\sum_{k=1}^{2} \Phi_{k}\left(\Psi_{k},\left\langle\varphi(\cdot), \beta_{n_{k}}\right\rangle\right)_{k} \cdot \beta_{n_{k}}
$$

with $\beta_{n_{k}}=\left(\beta_{n_{k}}^{(1)}, \beta_{n_{k}}^{(2)}, \cdots \beta_{n_{k}}^{(n)}\right),\left\langle\varphi(\cdot), \beta_{n_{k}}\right\rangle=\left(\begin{array}{c}\left\langle\varphi(\cdot), \beta_{n_{k}}^{(1)}\right\rangle \\ \left\langle\varphi(\cdot), \beta_{n_{k}}^{(2)}\right\rangle \\ \vdots \\ \left\langle\varphi(\cdot), \beta_{n_{k}}^{(n)}\right\rangle\end{array}\right), c \cdot \beta_{n_{k}}=c_{1} \beta_{n_{k}}^{(1)}+c_{2} \beta_{n_{k}}^{(2)}+\cdots+$ $c_{n} \beta_{n_{k}}^{(n)}$ for $c=\left(c_{1}, c_{2}, \cdots, c_{n}\right)^{T} \in \mathcal{C}$.

Decompose $U^{t} \in \mathcal{C}_{0}^{1}$ according to (12) as

$$
U^{t}(\theta)=\sum_{k=1}^{2} \Phi_{k}(\theta) \widetilde{z}_{k}(t) \cdot \beta_{n_{k}}+y_{t}(\theta)
$$

where $\widetilde{z}_{k}(t)=\left(\Psi_{k},\left\langle U^{t}, \beta_{n_{k}}\right\rangle\right)_{k}$, and $y_{t} \in \mathcal{C}_{0}^{1} \bigcap \operatorname{Ker} \pi:=\mathcal{Q}^{1}$ for any $t$. Then in $\mathcal{B C}$ the system (9) is equivalent to the system

$$
\begin{aligned}
& \dot{z}=B z+\Psi(0)\left(\begin{array}{c}
\left\langle\widetilde{F}\left(\alpha, \sum_{k=1}^{2}\left(\Phi_{k} \widetilde{z}_{k}\right) \cdot \beta_{n_{k}}+y\right), \beta_{n_{1}}\right\rangle \\
\left\langle\widetilde{F}\left(\alpha, \sum_{k=1}^{2}\left(\Phi_{k} \widetilde{z}_{k}\right) \cdot \beta_{n_{k}}+y\right), \beta_{n_{2}}\right\rangle
\end{array}\right), \\
& \frac{\mathrm{d} y}{\mathrm{~d} t}=A_{1} y+(I-\pi) X_{0} \widetilde{F}\left(\alpha, \sum_{k=1}^{2}\left(\Phi_{k} \widetilde{z}_{k}\right) \cdot \beta_{n_{k}}+y\right),
\end{aligned}
$$

where $z=\left(\widetilde{z}_{1}, \widetilde{z}_{2}\right)^{T}:=\left(z_{1}, z_{2}, z_{3}, z_{4}\right)^{T}, B=\operatorname{diag}\left(\mathrm{i} \omega_{1},-\mathrm{i} \omega_{1}, \mathrm{i} \omega_{2},-\mathrm{i} \omega_{2}\right)$, and $A_{1}$ is the restriction of $A$ on $\mathcal{Q}^{1} \subset \operatorname{Ker} \pi \rightarrow \operatorname{Ker} \pi, A_{1} \varphi=A \varphi$ for $\varphi \in \mathcal{Q}^{1}$. 


\section{CENTER MANIFOLD REDUCTION AND NORMAL FORM}

In the previous section, we have calculated the basis of the center subspace and defined a projection onto it. Applying the center manifold theory [38, 53], we know there exists an invariant local center manifold of the equilibrium, which will be calculated approximately and be used to obtain the normal form.

\subsection{Center Manifold Reduction}

Consider the formal Taylor expansion

$$
\widetilde{F}(\alpha, \varphi)=\sum_{j \geq 2} \frac{1}{j !} \widetilde{F}_{j}(\alpha, \varphi),
$$

where $\widetilde{F}_{j}$ is the $j$ th Fréchet derivation of $\widetilde{F}$. Then (14) can be written as

$$
\begin{aligned}
& \dot{z}=B z+\sum_{j \geq 2} \frac{1}{j !} f_{j}^{1}(z, y, \alpha), \\
& \frac{\mathrm{d} y}{\mathrm{~d} t}=A_{1} y+\sum_{j \geq 2} \frac{1}{j !} f_{j}^{2}(z, y, \alpha),
\end{aligned}
$$

where $z=\left(z_{1}, z_{2}, z_{3}, z_{4}\right)^{T} \in \mathbb{C}^{4}, y \in \mathcal{Q}^{1}$, and $f_{j}=\left(f_{j}^{1}, f_{j}^{2}\right), j \geq 2$, are defined by

$$
\begin{aligned}
& f_{j}^{1}(z, y, \alpha)=\Psi(0)\left(\begin{array}{c}
\left\langle\widetilde{F}_{j}\left(\alpha,\left(\sum_{k=1}^{2} \Phi_{k} \widetilde{z}_{k}\right) \cdot \beta_{n_{k}}+y\right), \beta_{n_{1}}\right\rangle \\
\left\langle\widetilde{F}_{j}\left(\alpha,\left(\sum_{k=1}^{2} \Phi_{k} \widetilde{z}_{k}\right) \cdot \beta_{n_{k}}+y\right), \beta_{n_{2}}\right\rangle
\end{array}\right), \\
& f_{j}^{2}=(I-\pi) X_{0} \widetilde{F}_{j}\left(\alpha,\left(\sum_{k=1}^{2} \Phi_{k} \widetilde{z}_{k}\right) \cdot \beta_{n_{k}}+y\right) .
\end{aligned}
$$

Let us introduce the following notations as those in [18]: for a normed space $Y, V_{j}^{6}(Y)$ denotes the space of homogeneous polynomials of degree $j$ in 6 variables $z=\left(z_{1}, z_{2}, z_{3}, z_{4}\right)^{T}$, $\alpha=\left(\alpha_{1}, \alpha_{2}\right)^{T}$ with coefficients in $Y$,

$$
V_{j}^{6}(Y)=\left\{\sum_{|(q, l)|=j} c_{(q, l)} z^{q} \alpha^{l}:(q, l) \in \mathbb{N}_{0}^{6}, c_{(q, l)} \in Y\right\}
$$

and the norm $\left|\sum_{|(q, l)|=j} c_{(q, l)} z^{q} \alpha^{l}\right|=\sum_{|(q, l)|=j}\left|c_{(q, l)}\right|_{Y}$. Define the operator $M_{j}=\left(M_{j}^{1}, M_{j}^{2}\right), j \geq 2$ by

$$
\begin{aligned}
& M_{j}^{1}: V_{j}^{6}\left(\mathbb{C}^{4}\right) \rightarrow V_{j}^{6}\left(\mathbb{C}^{4}\right), \\
& \left(M_{j}^{1} p\right)(z, \alpha)=D_{z} p(z, \alpha) B z-B p(z, \alpha), \\
& M_{j}^{2}: V_{j}^{6}\left(\mathcal{Q}_{1}\right) \subset V_{j}^{6}(\operatorname{Ker} \pi) \rightarrow V_{j}^{6}(\operatorname{Ker} \pi), \\
& \left(M_{j}^{2} h\right)(z, \alpha)=D_{z} h(z, \alpha) B z-A_{1} h(z, \alpha) .
\end{aligned}
$$


According to [18], the normal forms are obtained by a recursive transformations of variables of the form

$$
(z, y, \alpha)=(\widehat{z}, \widehat{y}, \alpha)+\frac{1}{j !}\left(U_{j}^{1}(\widehat{z}, \alpha), U_{j}^{2}(\widehat{z}, \alpha), 0\right),
$$

with $U_{j}=\left(U_{j}^{1}, U_{j}^{2}\right) \in V_{j}^{6}\left(\mathbb{C}^{4}\right) \times V_{j}^{6}\left(\mathcal{Q}_{1}\right)$. This recursive process transforms (15) into the normal form

$$
\begin{aligned}
& \dot{z}=B z+\sum_{j \geq 2} \frac{1}{j !} g_{j}^{1}(z, y, \alpha), \\
& \frac{d y}{d t}=A_{1} y+\sum_{j \geq 2} \frac{1}{j !} g_{j}^{2}(z, y, \alpha),
\end{aligned}
$$

where $g_{j}=\left(g_{j}^{1}, g_{j}^{2}\right), j \geq 2$, are the new terms of order $j$, given by

$$
g_{j}(z, y, \alpha)=\bar{f}_{j}(z, y, \alpha)-M_{j} U_{j}(z, \alpha)
$$

and $U_{j} \in V_{j}^{6}\left(\mathbb{C}^{4}\right) \times V_{j}^{6}\left(\mathcal{Q}_{1}\right)$ are given by

$$
U_{j}(z, \alpha)=\left(M_{j}\right)^{-1} \mathbf{P}_{\operatorname{Im}\left(M_{j}^{1}\right) \times \operatorname{Im}\left(M_{j}^{2}\right)} \circ \bar{f}_{j}(z, 0, \alpha)
$$

where $\mathbf{P}$ is the projection operator, and $\bar{f}_{j}=\left(\bar{f}_{j}^{1}, \bar{f}_{j}^{2}\right)$ denote the terms of order $j$ in $(z, y)$ obtained after the computation of normal forms up to order $j-1$.

\subsection{Second Order Normal Form}

For simplification of notations, we write $z=\left(z_{1}, z_{2}, z_{3}, z_{4}\right)^{T}, \Phi(\theta)=\left(\Phi_{1}(\theta), \Phi_{2}(\theta)\right), z_{x}=$ $\left(z_{1} \gamma_{n_{1}}, z_{2} \gamma_{n_{1}}, z_{3} \gamma_{n_{2}}, z_{4} \gamma_{n_{2}}\right)^{T}$. (13) can be written as

$$
\begin{aligned}
& U^{t}(\theta) \quad\left(\begin{array}{c}
z_{1}(t) \\
z_{2}(t)
\end{array}\right) \cdot \beta_{n_{1}}+\Phi_{2}(\theta)\left(\begin{array}{c}
z_{3}(t) \\
z_{4}(t)
\end{array}\right) \cdot \beta_{n_{2}}+y_{t}(\theta) \\
& =\Phi_{1}(\theta)\left(\begin{array}{c}
z_{1}(t) \\
z_{2}(t)
\end{array}\right) \gamma_{n_{1}}+\Phi_{2}(\theta)\left(\begin{array}{c}
z_{3}(t) \\
z_{4}(t)
\end{array}\right) \gamma_{n_{2}}+y_{t}(\theta) \\
& =\Phi(\theta)\left(\begin{array}{c}
z_{1}(t) \gamma_{n_{1}} \\
z_{2}(t) \gamma_{n_{1}} \\
z_{3}(t) \gamma_{n_{2}} \\
z_{4}(t) \gamma_{n_{2}}
\end{array}\right)+\left(\begin{array}{c}
y_{1 t}(\theta) \\
\vdots \\
y_{n t}(\theta)
\end{array}\right) \\
& =\phi_{1}(\theta) z_{1}(t) \gamma_{n_{1}}+\phi_{2}(\theta) z_{2}(t) \gamma_{n_{1}}+\phi_{3}(\theta) z_{3}(t) \gamma_{n_{2}}+\phi_{4}(\theta) z_{4}(t) \gamma_{n_{2}}+y_{t}(\theta) \\
& \triangleq \Phi(\theta) z_{x}(t)+y_{t}(\theta) .
\end{aligned}
$$


Then, by (14), (4) is equivalent to the system

$$
\begin{aligned}
& \dot{z}=B z+\Psi(0)\left(\begin{array}{c}
\left\langle\widetilde{F}\left(\alpha, \Phi z_{x}+y\right), \beta_{n_{1}}\right\rangle \\
\left\langle\widetilde{F}\left(\alpha, \Phi z_{x}+y\right), \beta_{n_{2}}\right\rangle
\end{array}\right), \\
& \frac{\mathrm{d} y}{\mathrm{~d} t}=A_{1} y+\left(I-\pi_{0}\right) X_{0} \widetilde{F}\left(\alpha, \Phi z_{x}+y\right) .
\end{aligned}
$$

When $\omega_{1}: \omega_{2} \neq m: n$ for $m, n \in \mathbb{N}$ and $1 \leq m, n \leq 3$, by (17), it is easy to verify that

$$
\begin{aligned}
& M_{j}^{1}\left(z^{p} \alpha^{\iota} e_{\xi}\right)=D_{z}\left(z^{p} \alpha^{\iota} e_{\xi}\right) B z-B z^{p} \alpha^{\iota} e_{\xi} \\
& =\left\{\begin{array}{l}
\left(i \omega_{1} p_{1}-i \omega_{1} p_{2}+i \omega_{2} p_{3}-i \omega_{2} p_{4}+(-1)^{\xi} i \omega_{1}\right) z^{p} \alpha^{\iota} e_{\xi}, \xi=1,2, \\
\left(i \omega_{1} p_{1}-i \omega_{1} p_{2}+i \omega_{2} p_{3}-i \omega_{2} p_{4}+(-1)^{\xi} i \omega_{2}\right) z^{p} \alpha^{\iota} e_{\xi}, \xi=3,4,
\end{array}\right.
\end{aligned}
$$

where $\xi=1,2,3,4, e_{1}=(1,0,0,0)^{T}, e_{2}=(0,1,0,0)^{T}, e_{3}=(0,0,1,0)^{T}, e_{4}=(0,0,0,1)^{T}$, $z^{p}=z_{1}^{p_{1}} z_{2}^{p_{2}} z_{3}^{p_{3}} z_{4}^{p_{4}}, \alpha^{\iota}=a_{1}^{\iota_{1}} a_{2}^{\iota_{2}}, p_{1}, p_{2}, p_{3}, p_{4}, \iota_{1}, \iota_{2} \in \mathbb{N}_{0}, p_{1}+p_{2}+p_{3}+p_{4}+\iota_{1}+\iota_{2}=j$. Therefore,

$$
\operatorname{Im}\left(M_{2}^{1}\right)^{c}=\operatorname{span}\left\{\left(\begin{array}{c}
\alpha_{i} z_{1} \\
0 \\
0 \\
0
\end{array}\right),\left(\begin{array}{c}
0 \\
\alpha_{i} z_{2} \\
0 \\
0
\end{array}\right),\left(\begin{array}{c}
0 \\
0 \\
\alpha_{i} z_{3} \\
0
\end{array}\right),\left(\begin{array}{c}
0 \\
0 \\
0 \\
\alpha_{i} z_{4}
\end{array}\right)\right\}, i=1,2 .
$$

Thus, the second order normal form of (44) on the center manifold of the origin near $\mu=\mu_{0}$ has the following form

$$
\dot{z}=B z+\frac{1}{2 !} g_{2}^{1}(z, 0, \alpha)+\cdots,
$$

with $g_{2}^{1}(z, 0, \alpha)=\operatorname{Proj}_{\operatorname{Im}\left(M_{2}^{1}\right)^{c}} f_{2}^{1}(z, 0, \alpha)$.

Write the Taylor expansions of $L\left(\mu_{0}+\alpha\right)$ and $D\left(\mu_{0}+\alpha\right)$ as follows

$$
\begin{gathered}
L\left(\alpha+\mu_{0}\right)=L_{0}+\alpha_{1} L_{1}^{(1,0)}+\alpha_{2} L_{1}^{(0,1)}+\frac{1}{2}\left(\alpha_{1}^{2} L_{2}^{(2,0)}+2 \alpha_{1} \alpha_{2} L_{2}^{(1,1)}+\alpha_{2}^{2} L_{2}^{(0,2)}\right)+\cdots \\
D\left(\alpha+\mu_{0}\right)=D_{0}+\alpha_{1} D_{1}^{(1,0)}+\alpha_{2} D_{1}^{(0,1)}+\frac{1}{2}\left(\alpha_{1}^{2} D_{2}^{(2,0)}+2 \alpha_{1} \alpha_{2} D_{2}^{(1,1)}+\alpha_{2}^{2} D_{2}^{(0,2)}\right)+\cdots
\end{gathered}
$$

Thus, the second order term of $\widetilde{F}\left(\alpha, U^{t}\right)$ is

$$
\widetilde{F}_{2}\left(\alpha, U^{t}\right)=2\left(\alpha_{1} D_{1}^{(1,0)}+\alpha_{2} D_{1}^{(0,1)}\right) \Delta U^{t}(0)+2\left(\alpha_{1} L_{1}^{(1,0)}+\alpha_{2} L_{1}^{(0,1)}\right) U^{t}+F_{2}\left(\alpha, U^{t}\right) .
$$

By (20), we can write

$$
\begin{aligned}
\widetilde{F}_{2}(z, y, \alpha) & =\widetilde{F}_{2}\left(\alpha, \Phi z_{x}+y\right) \\
& =2\left(\alpha_{1} D_{1}^{(1,0)}+\alpha_{2} D_{1}^{(0,1)}\right) \Delta\left(\Phi(0) z_{x}+y(0)\right) \\
& +2\left(\alpha_{1} L_{1}^{(1,0)}+\alpha_{2} L_{1}^{(0,1)}\right)\left(\Phi z_{x}+y\right)+F_{2}\left(\alpha, \Phi z_{x}+y\right) .
\end{aligned}
$$


Recalling assumptions $F(\mu, 0)=0, D_{\phi} F(\mu, 0)=0$, we have $F_{2}\left(\alpha, \Phi z_{x}+y\right)=F_{2}\left(0, \Phi z_{x}+y\right)$, which can be expressed by

$$
\begin{aligned}
F_{2}\left(0, \Phi z_{x}+y\right) & =\sum_{q_{1}+q_{2}+q_{3}+q_{4}=2} F_{q_{1} q_{2} q_{3} q_{4}} \gamma_{n_{1}}^{q_{1}+q_{2}}(x) \gamma_{n_{2}}^{q_{3}+q_{4}}(x) z_{1}^{q_{1}} z_{2}^{q_{2}} z_{3}^{q_{3}} z_{4}^{q_{4}} \\
& +S_{2}\left(\Phi z_{x}, y\right)+o\left(|y|^{2}\right) .
\end{aligned}
$$

Here $S_{2}\left(\Phi z_{x}, y\right)$ represents the linear terms of $y$, which can be calculated by $D_{\phi} F_{2}\left(0, \Phi z_{x}+\right.$ y) $\left.\right|_{y=0}(y)$.

From (16), (20), and (23), we have

$$
\frac{1}{2 !} f_{2}^{1}(z, 0, \alpha)=\frac{1}{2 !} \Psi(0)\left(\begin{array}{c}
\left\langle\widetilde{F}_{2}(z, 0, \alpha), \beta_{n_{1}}\right\rangle \\
\left\langle\widetilde{F}_{2}(z, 0, \alpha), \beta_{n_{2}}\right\rangle
\end{array}\right) .
$$

According to (22) and the fact that

$$
\int_{0}^{l \pi} \gamma_{n_{1}}^{2} d x=\int_{0}^{l \pi} \gamma_{n_{2}}^{2} d x=1
$$

we obtain

$$
\frac{1}{2 !} g_{2}^{1}(z, 0, \alpha)=\frac{1}{2 !} \operatorname{Proj}_{\operatorname{Im}\left(M_{2}^{1}\right)^{c}} f_{2}^{1}(z, 0, \alpha)=\left(\begin{array}{c}
B_{11} \alpha_{1} z_{1}+B_{21} \alpha_{2} z_{1} \\
\overline{B_{11}} \alpha_{1} z_{2}+\overline{B_{21}} \alpha_{2} z_{2} \\
B_{13} \alpha_{1} z_{3}+B_{23} \alpha_{2} z_{3} \\
\overline{B_{13}} \alpha_{1} z_{4}+\overline{B_{23}} \alpha_{2} z_{4}
\end{array}\right)
$$

with

$$
\begin{aligned}
& B_{11}=\psi_{1}(0)\left(-\frac{n_{1}^{2}}{l^{2}} D_{1}^{(1,0)} \phi_{1}(0)+L_{1}^{(1,0)} \phi_{1}\right), \\
& B_{21}=\psi_{1}(0)\left(-\frac{n_{1}^{2}}{l^{2}} D_{1}^{(0,1)} \phi_{1}(0)+L_{1}^{(0,1)} \phi_{1}\right), \\
& B_{13}=\psi_{3}(0)\left(-\frac{n_{2}^{2}}{l^{2}} D_{1}^{(1,0)} \phi_{3}(0)+L_{1}^{(1,0)} \phi_{3}\right), \\
& B_{23}=\psi_{3}(0)\left(-\frac{n_{2}^{2}}{l^{2}} D_{1}^{(0,1)} \phi_{3}(0)+L_{1}^{(0,1)} \phi_{3}\right) .
\end{aligned}
$$

Remark 1. For many systems with discrete time delays, if there are $m$ discrete delays in system (4), we can write

$$
L(\alpha, \varphi)=A(\alpha) \varphi(0)+G_{1}(\alpha) \varphi\left(-r_{1}\right)+G_{2}(\alpha) \varphi\left(-r_{2}\right)+\cdots+G_{m}(\alpha) \varphi\left(-r_{m}\right)
$$

and thus (26) can be calculated explicitly as follows

$$
\begin{aligned}
& B_{11}=\psi_{1}(0)\left(-\frac{n_{1}^{2}}{l^{2}} D_{1}^{(1,0)} \phi_{1}(0)+A_{1}^{(1,0)} \phi_{1}(0)+G_{11}^{(1,0)} \phi_{1}\left(-r_{1}\right)+\cdots+G_{m 1}^{(1,0)} \phi_{1}\left(-r_{m}\right)\right), \\
& B_{21}=\psi_{1}(0)\left(-\frac{n_{1}^{2}}{l^{2}} D_{1}^{(0,1)} \phi_{1}(0)+A_{1}^{(0,1)} \phi_{1}(0)+G_{11}^{(0,1)} \phi_{1}\left(-r_{1}\right)+\cdots+G_{m 1}^{(0,1)} \phi_{1}\left(-r_{m}\right)\right), \\
& B_{13}=\psi_{3}(0)\left(-\frac{n_{2}^{2}}{l^{2}} D_{1}^{(1,0)} \phi_{3}(0)+A_{1}^{(1,0)} \phi_{3}(0)+G_{11}^{(1,0)} \phi_{3}\left(-r_{1}\right)+\cdots+G_{m 1}^{(1,0)} \phi_{3}\left(-r_{m}\right)\right), \\
& B_{23}=\psi_{3}(0)\left(-\frac{n_{2}^{2}}{l^{2}} D_{1}^{(0,1)} \phi_{3}(0)+A_{1}^{(0,1)} \phi_{3}(0)+G_{11}^{(0,1)} \phi_{3}\left(-r_{1}\right)+\cdots+G_{m 1}^{(0,1)} \phi_{3}\left(-r_{m}\right)\right) .
\end{aligned}
$$




\subsection{Third Order Normal Form}

To find the third order normal form of the double Hopf singularity, neglecting the high order terms of the perturbation parameters and noticing the assumption $\left(H_{2}\right)$, we have

$$
\begin{aligned}
& \operatorname{Im}\left(M_{3}^{1}\right)^{c}=\operatorname{span}\left\{\left(\begin{array}{c}
z_{1}^{2} z_{2} \\
0 \\
0 \\
0
\end{array}\right),\left(\begin{array}{c}
z_{1} z_{3} z_{4} \\
0 \\
0 \\
0
\end{array}\right),\left(\begin{array}{c}
0 \\
z_{1} z_{2}^{2} \\
0 \\
0
\end{array}\right),\left(\begin{array}{c}
0 \\
z_{2} z_{3} z_{4} \\
0 \\
0
\end{array}\right),\left(\begin{array}{c}
0 \\
0 \\
z_{3}^{2} z_{4} \\
0
\end{array}\right),\right. \\
& \left.\left(\begin{array}{c}
0 \\
0 \\
z_{1} z_{2} z_{3} \\
0
\end{array}\right),\left(\begin{array}{c}
0 \\
0 \\
0 \\
z_{3} z_{4}^{2}
\end{array}\right),\left(\begin{array}{c}
0 \\
0 \\
0 \\
z_{1} z_{2} z_{4}
\end{array}\right)\right\}
\end{aligned}
$$

According to [18], the normal form up to the third order is given by

$$
\dot{z}=B z+\frac{1}{2 !} g_{2}^{1}(z, 0, \alpha)+\frac{1}{3 !} g_{3}^{1}(z, 0,0)+\cdots,
$$

where $g_{3}^{1}(z, 0,0)=\operatorname{Proj}_{\operatorname{Ker}\left(M_{3}^{1}\right)} \bar{f}_{3}^{1}(z, 0,0)$, with

$$
\begin{aligned}
\bar{f}_{3}^{1}(z, 0,0)= & f_{3}^{1}(z, 0,0)+\frac{3}{2}\left[D_{z} f_{2}^{1}(z, 0,0) U_{2}^{1}(z, 0)\right. \\
& \left.+D_{y} f_{2}^{1}(z, 0,0) U_{2}^{2}(z, 0)-D_{z} U_{2}^{1}(z, 0) g_{2}^{1}(z, 0,0)\right],
\end{aligned}
$$

with $\left(U_{2}^{1}(z, \alpha), U_{2}^{2}(z, \alpha)\right) \in V_{j}^{6}\left(\mathbb{C}^{4}\right) \times V_{j}^{6}\left(\mathcal{Q}_{1}\right)$ given by (19). After the calculation of the second order normal forms, we obtain the third order $\bar{f}_{3}^{1}(z, 0,0)$ given by (27). Noticing that $g_{2}^{1}(z, 0,0)=0$, we divide the computation of the third term $g_{3}^{1}(z, 0,0)=\operatorname{Proj}_{\operatorname{Im}\left(M_{3}^{1}\right)} \bar{f}_{3}^{1}(z, 0,0)$ into the following three parts

$$
\begin{gathered}
\operatorname{Proj}_{\operatorname{Im}\left(M_{3}^{1}\right)^{c}}\left(f_{3}^{1}(z, 0,0)\right), \\
\operatorname{Proj}_{\operatorname{Im}\left(M_{3}^{1}\right)^{c}}\left(D_{z} f_{2}^{1}(z, 0,0) U_{2}^{1}(z, 0)\right),
\end{gathered}
$$

and

$$
\operatorname{Proj}_{\operatorname{Im}\left(M_{3}^{1}\right)^{c}}\left(D_{y} f_{2}^{1}(z, 0,0) U_{2}^{2}(z, 0)\right),
$$

which will be calculated in the following part.

Step 1 The calculation of $\operatorname{Proj}_{\operatorname{Im}\left(M_{3}^{1}\right)^{c}}\left(f_{3}^{1}(z, 0,0)\right)$.

Calculating the third order Fréchet derivative of $\widetilde{F}\left(0, \Phi z_{x}\right)$ as follows

$$
\widetilde{F}_{3}\left(0, \Phi z_{x}\right)=\sum_{q_{1}+q_{2}+q_{3}+q_{4}=3} F_{q_{1} q_{2} q_{3} q_{4}} \gamma_{n_{1}}^{q_{1}+q_{2}}(x) \gamma_{n_{2}}^{q_{3}+q_{4}}(x) z_{1}^{q_{1}} z_{2}^{q_{2}} z_{3}^{q_{3}} z_{4}^{q_{4}},
$$


we have

$$
\begin{aligned}
& f_{3}^{1}(z, 0,0)=\Psi(0)\left(\begin{array}{c}
\left\langle\widetilde{F}_{3}\left(0, \Phi z_{x}\right), \beta_{n_{1}}\right\rangle \\
\left\langle\widetilde{F}_{3}\left(0, \Phi z_{x}\right), \beta_{n_{2}}\right\rangle
\end{array}\right) \\
& =\Psi(0)\left(\begin{array}{c}
\sum_{q_{1}+q_{2}+q_{3}+q_{4}=3} F_{q_{1} q_{2} q_{3} q_{4}} \int_{0}^{l \pi} \gamma_{n_{1}}^{q_{1}+q_{2}+1}(x) \gamma_{n_{2}}^{q_{3}+q_{4}}(x) d x z_{1}^{q_{1}} z_{2}^{q_{2}} z_{3}^{q_{3}} z_{4}^{q_{4}} \\
\sum_{q_{1}+q_{2}+q_{3}+q_{4}=3} F_{q_{1} q_{2} q_{3} q_{4}} \int_{0}^{l \pi} \gamma_{n_{1}}^{q_{1}+q_{2}}(x) \gamma_{n_{2}}^{q_{3}+q_{4}+1}(x) d x z_{1}^{q_{1}} z_{2}^{q_{2}} z_{3}^{q_{3}} z_{4}^{q_{4}}
\end{array}\right) .
\end{aligned}
$$

Thus,

$$
\frac{1}{3 !} \operatorname{Proj}_{\operatorname{Im}\left(M_{3}^{1}\right)^{c}}\left(f_{3}^{1}(z, 0,0)\right)=\left(\begin{array}{l}
C_{2100} z_{1}^{2} z_{2}+C_{1011} z_{1} z_{3} z_{4} \\
\overline{C_{2100}} z_{1} z_{2}^{2}+\overline{C_{1011}} z_{2} z_{3} z_{4} \\
C_{0021} z_{3}^{2} z_{4}+C_{1110} z_{1} z_{2} z_{3} \\
\overline{C_{0021}} z_{3} z_{4}^{2}+\overline{C_{1110}} z_{1} z_{2} z_{4}
\end{array}\right),
$$

where

$$
\begin{aligned}
C_{2100} & =\frac{1}{6} \psi_{1}(0) F_{2100} \gamma_{40}, C_{1011}=\frac{1}{6} \psi_{1}(0) F_{1011} \gamma_{22}, \\
C_{0021} & =\frac{1}{6} \psi_{3}(0) F_{0021} \gamma_{04}, C_{1110}=\frac{1}{6} \psi_{3}(0) F_{1110} \gamma_{22} .
\end{aligned}
$$

Here $\gamma_{i j}=\int_{0}^{l \pi} \gamma_{n_{1}}^{i}(x) \gamma_{n_{2}}^{j}(x) d x$, and

$$
\begin{aligned}
& \int_{0}^{l \pi} \gamma_{n_{k}}^{4}(x) d x=\left\{\begin{array}{c}
\frac{1}{l \pi}, n_{k}=0, \quad(k=1,2), \\
\frac{3}{2 l \pi}, n_{k} \neq 0,
\end{array}\right. \\
& \int_{0}^{l \pi} \gamma_{n_{1}}^{2}(x) \gamma_{n_{2}}^{2}(x) d x=\left\{\begin{array}{cl}
\frac{1}{l \pi}, & n_{1}=0, n_{2}=0, \\
\frac{1}{l \pi}, & n_{1}=0, n_{2} \neq 0, \\
\frac{1}{l \pi}, & n_{1} \neq 0, n_{2} \neq 0, n_{1} \neq n_{2}, \\
\frac{3}{2 l \pi}, & n_{1}=n_{2} \neq 0 .
\end{array}\right.
\end{aligned}
$$

Step $2 \operatorname{Proj}_{\operatorname{Im}\left(M_{3}^{1}\right)}\left(D_{z} f_{2}^{1}(z, 0,0) U_{2}^{1}(z, 0)\right)$.

From (16), (20), (23) and (24), we can write $f_{2}^{1}(z, 0,0)=\left(f_{2}^{1(1)}, f_{2}^{1(2)}, f_{2}^{1(3)}, f_{2}^{1(4)}\right)^{T}$ as

$$
\begin{aligned}
& f_{2}^{1}(z, 0,0)=\Psi(0)\left(\begin{array}{c}
\left\langle F_{2}\left(0, \Phi z_{x}\right), \beta_{n_{1}}\right\rangle \\
\left\langle F_{2}\left(0, \Phi z_{x}\right), \beta_{n_{2}}\right\rangle
\end{array}\right) \\
& =\Psi(0)\left(\begin{array}{c}
\sum_{q_{1}+q_{2}+q_{3}+q_{4}=2} F_{q_{1} q_{2} q_{3} q_{4}} \int_{0}^{l \pi} \gamma_{n_{1}}^{q_{1}+q_{2}+1}(x) \gamma_{n_{2}}^{q_{3}+q_{4}}(x) d x z_{1}^{q_{1}} z_{2}^{q_{2}} z_{3}^{q_{3}} z_{4}^{q_{4}} \\
\sum_{q_{1}+q_{2}+q_{3}+q_{4}=2} F_{q_{1} q_{2} q_{3} q_{4}} \int_{0}^{l \pi} \gamma_{n_{1}}^{q_{1}+q_{2}}(x) \gamma_{n_{2}}^{q_{3}+q_{4}+1}(x) d x z_{1}^{q_{1}} z_{2}^{q_{2}} z_{3}^{q_{3}} z_{4}^{q_{4}}
\end{array}\right),
\end{aligned}
$$

where

$$
\begin{array}{r}
f_{2}^{1(1)}=\psi_{1}(0)\left(F_{2000} z_{1}^{2} \beta_{30}+F_{1100} z_{1} z_{2} \beta_{30}+F_{1010} z_{1} z_{3} \beta_{21}+F_{1001} z_{1} z_{4} \beta_{21}+F_{0200} z_{2}^{2} \beta_{30}\right. \\
\left.+F_{0110} z_{2} z_{3} \beta_{21}+F_{0101} z_{2} z_{4} \beta_{21}+F_{0020} z_{3}^{2} \beta_{12}+F_{0011} z_{3} z_{4} \beta_{12}+F_{0002} z_{4}^{2} \beta_{12}\right)
\end{array}
$$




$$
\begin{array}{r}
f_{2}^{1(2)}=\psi_{2}(0)\left(F_{2000} z_{1}^{2} \beta_{30}+F_{1100} z_{1} z_{2} \beta_{30}+F_{1010} z_{1} z_{3} \beta_{21}+F_{1001} z_{1} z_{4} \beta_{21}+F_{0200} z_{2}^{2} \beta_{30}\right. \\
\left.+F_{0110} z_{2} z_{3} \beta_{21}+F_{0101} z_{2} z_{4} \beta_{21}+F_{0020} z_{3}^{2} \beta_{12}+F_{0011} z_{3} z_{4} \beta_{12}+F_{0002} z_{4}^{2} \beta_{12}\right) \\
f_{2}^{1(3)}=\psi_{3}(0)\left(F_{2000} z_{1}^{2} \beta_{21}+F_{1100} z_{1} z_{2} \beta_{21}+F_{1010} z_{1} z_{3} \beta_{12}+F_{1001} z_{1} z_{4} \beta_{12}+F_{0200} z_{2}^{2} \beta_{21}\right. \\
\left.\quad+F_{0110} z_{2} z_{3} \beta_{12}+F_{0101} z_{2} z_{4} \beta_{12}+F_{0020} z_{3}^{2} \beta_{03}+F_{0011} z_{3} z_{4} \beta_{03}+F_{0002} z_{4}^{2} \beta_{03}\right) \\
f_{2}^{1(4)}=\psi_{4}(0)\left(F_{2000} z_{1}^{2} \beta_{21}+F_{1100} z_{1} z_{2} \beta_{21}+F_{1010} z_{1} z_{3} \beta_{12}+F_{1001} z_{1} z_{4} \beta_{12}+F_{0200} z_{2}^{2} \beta_{21}\right. \\
+ \\
\left.+F_{0110} z_{2} z_{3} \beta_{12}+F_{0101} z_{2} z_{4} \beta_{12}+F_{0020} z_{3}^{2} \beta_{03}+F_{0011} z_{3} z_{4} \beta_{03}+F_{0002} z_{4}^{2} \beta_{03}\right),
\end{array}
$$

and

$$
\begin{aligned}
& f_{2000}^{1(1)}=\psi_{1}(0) F_{2000} \beta_{30}, f_{2000}^{1(2)}=\psi_{2}(0) F_{2000} \beta_{30}, \\
& f_{2000}^{1(3)}=\psi_{3}(0) F_{2000} \beta_{21}, f_{2000}^{1(4)}=\psi_{4}(0) F_{2000} \beta_{21}, \\
& f_{1100}^{1(1)}=\psi_{1}(0) F_{1100} \beta_{30}, f_{1100}^{1(2)}=\psi_{2}(0) F_{1100} \beta_{30}, \\
& f_{1100}^{1(3)}=\psi_{3}(0) F_{1100} \beta_{21}, f_{1100}^{1(4)}=\psi_{4}(0) F_{1100} \beta_{21}, \\
& f_{1010}^{1(1)}=\psi_{1}(0) F_{1010} \beta_{21}, f_{1010}^{1(2)}=\psi_{2}(0) F_{1010} \beta_{21}, \\
& f_{1010}^{1(3)}=\psi_{3}(0) F_{1010} \beta_{12}, f_{1010}^{1(4)}=\psi_{4}(0) F_{1010} \beta_{12}, \\
& f_{1001}^{1(1)}=\psi_{1}(0) F_{1001} \beta_{21}, f_{1001}^{1(2)}=\psi_{2}(0) F_{1001} \beta_{21}, \\
& f_{1001}^{1(3)}=\psi_{3}(0) F_{1001} \beta_{12}, f_{1001}^{1(4)}=\psi_{4}(0) F_{1001} \beta_{12}, \\
& f_{0200}^{1(1)}=\psi_{1}(0) F_{0200} \beta_{30}, f_{0200}^{1(2)}=\psi_{2}(0) F_{0200} \beta_{30}, \\
& f_{0200}^{1(3)}=\psi_{3}(0) F_{0200} \beta_{21}, f_{0200}^{1(4)}=\psi_{4}(0) F_{0200} \beta_{21}, \\
& f_{0110}^{1(1)}=\psi_{1}(0) F_{0110} \beta_{21}, f_{0110}^{1(2)}=\psi_{2}(0) F_{0110} \beta_{21}, \\
& f_{0110}^{1(3)}=\psi_{3}(0) F_{0110} \beta_{12}, f_{0110}^{1(4)}=\psi_{4}(0) F_{0110} \beta_{12}, \\
& f_{0101}^{1(1)}=\psi_{1}(0) F_{0101} \beta_{21}, f_{0101}^{1(2)}=\psi_{2}(0) F_{0101} \beta_{21}, \\
& f_{0101}^{1(3)}=\psi_{3}(0) F_{0101} \beta_{12}, f_{0101}^{1(4)}=\psi_{4}(0) F_{0101} \beta_{12}, \\
& f_{0020}^{1(1)}=\psi_{1}(0) F_{0020} \beta_{12}, f_{0020}^{1(2)}=\psi_{2}(0) F_{0020} \beta_{12}, \\
& f_{0020}^{1(3)}=\psi_{3}(0) F_{0020} \beta_{03}, f_{0020}^{1(4)}=\psi_{4}(0) F_{0020} \beta_{03}, \\
& f_{0011}^{1(1)}=\psi_{1}(0) F_{0011} \beta_{12}, f_{0011}^{1(2)}=\psi_{2}(0) F_{0011} \beta_{12}, \\
& f_{0011}^{1(3)}=\psi_{3}(0) F_{0011} \beta_{03}, f_{0011}^{1(4)}=\psi_{4}(0) F_{0011} \beta_{03}, \\
& f_{0002}^{1(1)}=\psi_{1}(0) F_{0002} \beta_{12}, f_{0002}^{1(2)}=\psi_{3}(0) F_{0002} \beta_{03}, f_{0002}^{1(4)}=F_{4}(0) F_{0002} \beta_{03},
\end{aligned}
$$


with $\beta_{i j}=\int_{0}^{l \pi} \gamma_{n_{1}}^{i}(x) \gamma_{n_{2}}^{j}(x) d x$, and

$$
\begin{aligned}
& \int_{0}^{l \pi} \gamma_{n_{k}}^{3}(x) d x=\left\{\begin{array}{ll}
\sqrt{\frac{1}{l \pi}}, & n_{k}=0, \\
0, & n_{k} \neq 0,
\end{array} \quad(k=1,2),\right. \\
& \int_{0}^{l \pi} \gamma_{n_{1}}^{2}(x) \gamma_{n_{2}}(x) d x=\left\{\begin{array}{cc}
\sqrt{\frac{1}{l \pi}}, & n_{1}=0, n_{2}=0, \\
0, & n_{1}=0, n_{2} \neq 0, \\
\sqrt{\frac{1}{2 l \pi}}, & n_{1} \neq 0, n_{2} \neq 0, n_{2}=2 n_{1},
\end{array}\right. \\
& \int_{0}^{l \pi} \gamma_{n_{1}}(x) \gamma_{n_{2}}^{2}(x) d x=\left\{\begin{array}{cc}
0, & n_{1} \neq 0, n_{2} \neq 0, n_{2} \neq 2 n_{1}, \\
\sqrt{\frac{1}{l \pi}}, & n_{1}=0, n_{2}=0, \\
\sqrt{\frac{1}{l \pi}}, & n_{1}=0, n_{2} \neq 0, \\
0, & n_{1} \neq 0, n_{2} \neq 0 .
\end{array}\right.
\end{aligned}
$$

Combining with (21) and (29), we can calculate $U_{2}^{1}(z, 0)=\left(U_{2}^{1(1)}, U_{2}^{1(2)}, U_{2}^{1(3)}, U_{2}^{1(4)}\right)^{T}$ from the following formula

$$
U_{2}^{1}(z, 0)=\left(M_{2}^{1}\right)^{-1} \operatorname{Proj}_{\operatorname{Im}\left(M_{2}^{1}\right)} f_{2}^{1}(z, 0,0)
$$

Then, we have

$$
\begin{aligned}
U_{2}^{1(1)}= & \frac{1}{i \omega_{1}} f_{2000}^{1(1)} z_{1}^{2}+\frac{1}{-i \omega_{1}} f_{1100}^{1(1)} z_{1} z_{2}+\frac{1}{i \omega_{2}} f_{1010}^{1(1)} z_{1} z_{3}+\frac{1}{-i \omega_{2}} f_{1001}^{1(1)} z_{1} z_{4} \\
& +\frac{1}{-3 i \omega_{1}} f_{0200}^{1(1)} z_{2}^{2}+\frac{1}{-2 i \omega_{1}+i \omega_{2}} f_{0110}^{1(1)} z_{2} z_{3}+\frac{1}{-2 i \omega_{1}-i \omega_{2}} f_{0101}^{1(1)} z_{2} z_{4} \\
& +\frac{1}{2 i \omega_{2}-i \omega_{1}} f_{0020}^{1(1)} z_{3}^{2}+\frac{1}{-i \omega_{1}} f_{0011}^{1(1)} z_{3} z_{4}+\frac{1}{-2 i \omega_{2}-i \omega_{1}} f_{0002}^{1(1)} z_{4}^{2} \\
U_{2}^{1(2)}= & \frac{1}{3 i \omega_{1}} f_{2000}^{1(2)} z_{1}^{2}+\frac{1}{i \omega_{1}} f_{1100}^{1(2)} z_{1} z_{2}+\frac{1}{2 i \omega_{1}+i \omega_{2}} f_{1010}^{1(2)} z_{1} z_{3} \\
& +\frac{1}{2 i \omega_{1}-i \omega_{2}} f_{1001}^{1(2)} z_{1} z_{4}+\frac{1}{-i \omega_{1}} f_{0200}^{1(2)} z_{2}^{2}+\frac{1}{i \omega_{2}} f_{0110}^{1(2)} z_{2} z_{3}+\frac{1}{-i \omega_{2}} f_{0101}^{1(2)} z_{2} z_{4} \\
& +\frac{1}{2 i \omega_{2}+i \omega_{1}} f_{0020}^{1(2)} z_{3}^{2}+\frac{1}{i \omega_{1}} f_{0011}^{1(2)} z_{3} z_{4}+\frac{1}{-2 i \omega_{2}+i \omega_{1}} f_{0002}^{1(2)} z_{4}^{2}, \\
U_{2}^{1(3)}= & \frac{1}{2 i \omega_{1}-i \omega_{2}} f_{2000}^{1(3)} z_{1}^{2}+\frac{1}{-i \omega_{2}} f_{1100}^{1(3)} z_{1} z_{2}+\frac{1}{i \omega_{1}} f_{1010}^{1(3)} z_{1} z_{3}+\frac{1}{i \omega_{1}-2 i \omega_{2}} f_{1001}^{1(3)} z_{1} z_{4} \\
+ & \frac{1}{-2 i \omega_{1}-i \omega_{2}} f_{0200}^{1(3)} z_{2}^{2}+\frac{1}{-i \omega_{1}} f_{0110}^{1(3)} z_{2} z_{3}+\frac{1}{-i \omega_{1}-2 i \omega_{2}} f_{0101}^{1(3)} z_{2} z_{4} \\
+ & \frac{1}{i \omega_{2}} f_{0020}^{1(3)} z_{3}^{2}+\frac{1}{-i \omega_{2}} f_{0011}^{1(3)} z_{3} z_{4}+\frac{1}{-3 i \omega_{2}} f_{0002}^{1(3)} z_{4}^{2},
\end{aligned}
$$




$$
\begin{aligned}
U_{2}^{1(4)} & =\frac{1}{2 i \omega_{1}+i \omega_{2}} f_{2000}^{1(4)} z_{1}^{2}+\frac{1}{i \omega_{2}} f_{1100}^{1(4)} z_{1} z_{2}+\frac{1}{i \omega_{1}+2 i \omega_{2}} f_{1010}^{1(4)} z_{1} z_{3}+\frac{1}{i \omega_{1}} f_{1001}^{1(4)} z_{1} z_{4} \\
& +\frac{1}{-2 i \omega_{1}+i \omega_{2}} f_{0200}^{1(4)} z_{2}^{2}+\frac{1}{-i \omega_{1}+2 i \omega_{2}} f_{0110}^{1(4)} z_{2} z_{3}+\frac{1}{-i \omega_{1}} f_{0101}^{1(4)} z_{2} z_{4} \\
& +\frac{1}{3 i \omega_{2}} f_{0020}^{1(4)} z_{3}^{2}+\frac{1}{i \omega_{2}} f_{0011}^{1(4)} z_{3} z_{4}+\frac{1}{-i \omega_{2}} f_{0002}^{1(4)} z_{4}^{2} .
\end{aligned}
$$

Thus,

$$
\frac{1}{3 !} \operatorname{Proj}_{\operatorname{Im}\left(M_{3}^{1}\right)^{c}}\left(D_{z} f_{2}^{1}(z, 0,0) U_{2}^{1}(z, 0)\right)=\left(\begin{array}{c}
D_{2100} z_{1}^{2} z_{2}+D_{1011} z_{1} z_{3} z_{4} \\
\overline{D_{2100}} z_{1} z_{2}^{2}+\overline{D_{1011}} z_{2} z_{3} z_{4} \\
D_{0021} z_{3}^{2} z_{4}+D_{1110} z_{1} z_{2} z_{3} \\
\overline{D_{0021}} z_{3} z_{4}^{2}+\overline{D_{1110}} z_{1} z_{2} z_{4}
\end{array}\right),
$$

where

$$
\begin{aligned}
& D_{2100}=\frac{1}{6}\left(2 f_{2000}^{1(1)} \frac{1}{-i \omega_{1}} f_{1100}^{1(1)}+f_{1100}^{1(1)} \frac{1}{i \omega_{1}} f_{2000}^{1(1)}+f_{1100}^{1(1)} \frac{1}{i \omega_{1}} f_{1100}^{1(2)}\right. \\
& +2 f_{0200}^{1(1)} \frac{1}{3 i \omega_{1}} f_{2000}^{1(2)}+f_{1010}^{1(1)} \frac{1}{-i \omega_{2}} f_{1100}^{1(3)}+f_{0110}^{1(1)} \frac{1}{2 i \omega_{1}-i \omega_{2}} f_{2000}^{1(3)} \\
& \left.+f_{1001}^{1(1)} \frac{1}{i \omega_{2}} f_{1100}^{1(4)}+f_{0101}^{1(1)} \frac{1}{2 i \omega_{1}+i \omega_{2}} f_{2000}^{1(4)}\right), \\
& D_{1011}=\frac{1}{6}\left(2 f_{2000}^{1(1)} \frac{1}{-i \omega_{1}} f_{0011}^{1(1)}+f_{1010}^{1(1)} \frac{1}{-i \omega_{2}} f_{1001}^{1(1)}+f_{1001}^{1(1)} \frac{1}{i \omega_{2}} f_{1010}^{1(1)}\right. \\
& +f_{1100}^{1(1)} \frac{1}{i \omega_{1}} f_{0011}^{1(2)}+f_{0110}^{1(1)} \frac{1}{2 i \omega_{1}-i \omega_{2}} f_{1001}^{1(2)}+f_{0101}^{1(1)} \frac{1}{2 i \omega_{1}+i \omega_{2}} f_{1010}^{1(2)} \\
& +f_{1010}^{1(1)} \frac{1}{-i \omega_{2}} f_{0011}^{1(3)}+2 f_{0020}^{1(1)} \frac{1}{i \omega_{1}-2 i \omega_{2}} f_{1001}^{1(3)}+f_{0011}^{1(1)} \frac{1}{i \omega_{1}} f_{1010}^{1(3)} \\
& \left.+f_{1001}^{1(1)} \frac{1}{i \omega_{2}} f_{0011}^{1(4)}+f_{0011}^{1(1)} \frac{1}{i \omega_{1}} f_{1001}^{1(4)}+2 f_{0002}^{1(1)} \frac{1}{i \omega_{1}+2 i \omega_{2}} f_{1010}^{1(4)}\right), \\
& D_{0021}=\frac{1}{6}\left(f_{1010}^{1(3)} \frac{1}{-i \omega_{1}} f_{0011}^{1(1)}+f_{1001}^{1(3)} \frac{1}{2 i \omega_{2}-i \omega_{1}} f_{0020}^{1(1)}+f_{0110}^{1(3)} \frac{1}{i \omega_{1}} f_{0011}^{1(2)}\right. \\
& +f_{0101}^{1(3)} \frac{1}{2 i \omega_{2}+i \omega_{1}} f_{0020}^{1(2)}+2 f_{0020}^{1(3)} \frac{1}{-i \omega_{2}} f_{0011}^{1(3)}+f_{0011}^{1(3)} \frac{1}{i \omega_{2}} f_{0020}^{1(3)} \\
& \left.+f_{0011}^{1(3)} \frac{1}{i \omega_{2}} f_{0011}^{1(4)}+2 f_{0002}^{1(3)} \frac{1}{3 i \omega_{2}} f_{0020}^{1(4)}\right), \\
& D_{1110}=\frac{1}{6}\left(2 f_{2000}^{1(3)} \frac{1}{-2 i \omega_{1}+i \omega_{2}} f_{0110}^{1(1)}+f_{1100}^{1(3)} \frac{1}{i \omega_{2}} f_{1010}^{1(1)}+f_{1010}^{1(3)} \frac{1}{-i \omega_{1}} f_{1100}^{1(1)}\right. \\
& +f_{1100}^{1(3)} \frac{1}{i \omega_{2}} f_{0110}^{1(2)}+2 f_{0200}^{1(3)} \frac{1}{2 i \omega_{1}+i \omega_{2}} f_{1010}^{1(2)}+f_{0110}^{1(3)} \frac{1}{i \omega_{1}} f_{1100}^{1(2)} \\
& +f_{1010}^{1(3)} \frac{1}{-i \omega_{1}} f_{0110}^{1(3)}+f_{0110}^{1(3)} \frac{1}{i \omega_{1}} f_{1010}^{1(3)}+2 f_{0020}^{1(3)} \frac{1}{-i \omega_{2}} f_{1100}^{1(3)} \\
& \left.+f_{1001}^{1(3)} \frac{1}{-i \omega_{1}+2 i \omega_{2}} f_{0110}^{1(4)}+f_{0101}^{1(3)} \frac{1}{i \omega_{1}+2 i \omega_{2}} f_{1010}^{1(4)}+f_{0011}^{1(3)} \frac{1}{i \omega_{2}} f_{1100}^{1(4)}\right) .
\end{aligned}
$$

Step 3. The calculation of $\operatorname{Proj}_{\operatorname{Im}\left(M_{3}^{1}\right)^{c}}\left(D_{y} f_{2}^{1}(z, 0,0) U_{2}^{2}(z, 0)\right)$. 
First, we will calculate the Fréchet derivative $D_{y} f_{2}^{1}(z, 0,0): \mathcal{Q}_{1} \rightarrow X_{\mathbb{C}}$. From (23) and (24), $\widetilde{F}_{2}(z, y, 0)$ can be written as

$$
\begin{aligned}
& \widetilde{F}_{2}(z, y, 0)=S_{2}\left(\Phi z_{x}, y\right)+o\left(z^{2}, y^{2}\right) \\
& =S_{y z_{1}}(y) z_{1} \gamma_{n_{1}}+S_{y z_{2}}(y) z_{2} \gamma_{n_{1}}+S_{y z_{3}}(y) z_{3} \gamma_{n_{2}}+S_{y z_{4}}(y) z_{4} \gamma_{n_{2}}+o\left(z^{2}, y^{2}\right),
\end{aligned}
$$

where $S_{y z_{i}}(i=1,2,3,4)$ are linear operators from $\mathcal{Q}_{1}$ to $X_{\mathbb{C}}$.

Remark 2. Again if there are $m$ discrete delays in the system (4), we can get the explicit formula

$$
\begin{aligned}
S_{y z_{i}}(\varphi) & =\left(F_{y_{1}(0) z_{i}}, F_{y_{2}(0) z_{i}}, \cdots, F_{y_{n}(0) z_{i}}\right) \varphi(0) \\
& +\left(F_{y_{1}\left(-r_{1}\right) z_{i}}, F_{y_{2}\left(-r_{1}\right) z_{i}}, \cdots, F_{y_{n}\left(-r_{1}\right) z_{i}}\right) \varphi\left(-r_{1}\right)+\cdots \\
& +\left(F_{y_{1}\left(-r_{m}\right) z_{i}}, F_{y_{2}\left(-r_{m}\right) z_{i}}, \cdots, F_{y_{n}\left(-r_{m}\right) z_{i}}\right) \varphi\left(-r_{m}\right) .
\end{aligned}
$$

Now, we have

$$
D_{y} \widetilde{F}_{2}(z, 0,0)(\varphi)=S_{y z_{1}}(\varphi) z_{1} \gamma_{n_{1}}+S_{y z_{2}}(\varphi) z_{2} \gamma_{n_{1}}+S_{y z_{3}}(\varphi) z_{3} \gamma_{n_{2}}+S_{y z_{4}}(\varphi) z_{4} \gamma_{n_{2}}
$$

Let $U_{2}^{2}(z, 0)=h(z)=\sum_{j \geq 0} h_{j}(z) \cdot \beta_{j}(x)=\sum_{j \geq 0} h_{j}(z) \gamma_{j}(x)$ with

$$
h_{j}(z)=\left(\begin{array}{c}
h_{j}^{(1)}(z) \\
h_{j}^{(2)}(z) \\
\vdots \\
h_{j}^{(n)}(z)
\end{array}\right)=\sum_{q_{1}+q_{2}+q_{3}+q_{4}=2}\left(\begin{array}{c}
h_{j q_{1} q_{2} q_{3} q_{4}}^{(1)}(z) \\
h_{j q_{1} q_{2} q_{3} q_{4}}^{(2)}(z) \\
\vdots \\
h_{j q_{1} q_{2} q_{3} q_{4}}^{(n)}(z)
\end{array}\right) z_{1}^{q_{1}} z_{2}^{q_{2}} z_{3}^{q_{3}} z_{4}^{q_{4}}
$$

Thus,

$$
\begin{aligned}
& D_{y} f_{2}^{1}(z, 0,0)\left(U_{2}^{2}(z, 0)\right)=\Psi(0)\left(\begin{array}{c}
\left\langle D_{y} \widetilde{F}_{2}(z, 0,0)\left(U_{2}^{2}(z, 0)\right), \beta_{n_{1}}\right\rangle \\
\left\langle D_{y} \widetilde{F}_{2}(z, 0,0)\left(U_{2}^{2}(z, 0)\right), \beta_{n_{2}}\right\rangle
\end{array}\right) \\
& =\Psi(0)\left(\begin{array}{c}
\left\langle S_{y z_{1}}\left(\sum_{j \geq 0} h_{j} \gamma_{j}\right) \gamma_{n_{1}}, \beta_{n_{1}}\right\rangle z_{1}+\left\langle S_{y z_{2}}\left(\sum_{j \geq 0} h_{j} \gamma_{j}\right) \gamma_{n_{1}}, \beta_{n_{1}}\right\rangle z_{2} \\
+\left\langle S_{y z_{3}}\left(\sum_{j \geq 0} h_{j} \gamma_{j}\right) \gamma_{n_{2}}, \beta_{n_{1}}\right\rangle z_{3}+\left\langle S_{y z_{4}}\left(\sum_{j \geq 0} h_{j} \gamma_{j}\right) \gamma_{n_{2}}, \beta_{n_{1}}\right\rangle z_{4} \\
\left\langle S_{y z_{1}}\left(\sum_{j \geq 0} h_{j} \gamma_{j}\right) \gamma_{n_{1}}, \beta_{n_{2}}\right\rangle z_{1}+\left\langle S_{y z_{2}}\left(\sum_{j \geq 0} h_{j} \gamma_{j}\right) \gamma_{n_{1}}, \beta_{n_{2}}\right\rangle z_{2} \\
+\left\langle S_{y z_{3}}\left(\sum_{j \geq 0} h_{j} \gamma_{j}\right) \gamma_{n_{2}}, \beta_{n_{2}}\right\rangle z_{3}+\left\langle S_{y z_{4}}\left(\sum_{j \geq 0} h_{j} \gamma_{j}\right) \gamma_{n_{2}}, \beta_{n_{2}}\right\rangle z_{4}
\end{array}\right) \\
& =\Psi(0)\left(\begin{array}{c}
\sum_{j \geq 0}\left[b_{j n_{1} n_{1}} S_{y z_{1}}\left(h_{j}\right) z_{1}+b_{j n_{1} n_{1}} S_{y z_{2}}\left(h_{j}\right) z_{2}\right. \\
\left.+b_{j n_{2} n_{1}} S_{y z_{3}}\left(h_{j}\right) z_{3}+b_{j n_{2} n_{1}} S_{y z_{4}}\left(h_{j}\right) z_{4}\right] \\
\sum_{j \geq 0}\left[b_{j n_{1} n_{2}} S_{y z_{1}}\left(h_{j}\right) z_{1}+b_{j n_{1} n_{2}} S_{y z_{2}}\left(h_{j}\right) z_{2}\right. \\
\left.+b_{j n_{2} n_{2}} S_{y z_{3}}\left(h_{j}\right) z_{3}+b_{j n_{2} n_{2}} S_{y z_{4}}\left(h_{j}\right) z_{4}\right]
\end{array}\right)
\end{aligned}
$$


To give a clear expression of our derivation about the normal form, the rest part of derivation are divided into three cases: $n_{1}=n_{2}=0, n_{1}=0, n_{2} \neq 0$, and $n_{1} \neq 0, n_{2} \neq 0$.

Case 1 When $n_{1}=n_{2}=0$, in fact

$$
b_{j n_{1} n_{1}}=b_{j n_{1} n_{2}}=b_{j n_{2} n_{1}}=b_{j n_{2} n_{2}}=\int_{0}^{l \pi} \gamma_{j}(x) \gamma_{0}(x) \gamma_{0}(x) d x= \begin{cases}\frac{1}{\sqrt{l \pi}}, & j=0 \\ 0, & j \neq 0\end{cases}
$$

Then, obviously

$$
\begin{aligned}
& D_{y} f_{2}^{1}(z, 0,0)\left(U_{2}^{2}(z, 0)\right) \\
& =\frac{1}{\sqrt{l \pi}} \Psi(0)\left(\begin{array}{c}
S_{y z_{1}}\left(h_{0}\right) z_{1}+S_{y z_{2}}\left(h_{0}\right) z_{2}+S_{y z_{3}}\left(h_{0}\right) z_{3}+S_{y z_{4}}\left(h_{0}\right) z_{4} \\
S_{y z_{1}}\left(h_{0}\right) z_{1}+S_{y z_{2}}\left(h_{0}\right) z_{2}+S_{y z_{3}}\left(h_{0}\right) z_{3}+S_{y z_{4}}\left(h_{0}\right) z_{4}
\end{array}\right) \\
& =\frac{1}{\sqrt{l \pi}}\left(\begin{array}{c}
\psi_{1}(0)\left(S_{y z_{1}}\left(h_{0}\right) z_{1}+S_{y z_{2}}\left(h_{0}\right) z_{2}+S_{y z_{3}}\left(h_{0}\right) z_{3}+S_{y z_{4}}\left(h_{0}\right) z_{4}\right) \\
\psi_{2}(0)\left(S_{y z_{1}}\left(h_{0}\right) z_{1}+S_{y z_{2}}\left(h_{0}\right) z_{2}+S_{y z_{3}}\left(h_{0}\right) z_{3}+S_{y z_{4}}\left(h_{0}\right) z_{4}\right) \\
\psi_{3}(0)\left(S_{y z_{1}}\left(h_{0}\right) z_{1}+S_{y z_{2}}\left(h_{0}\right) z_{2}+S_{y z_{3}}\left(h_{0}\right) z_{3}+S_{y z_{4}}\left(h_{0}\right) z_{4}\right) \\
\psi_{4}(0)\left(S_{y z_{1}}\left(h_{0}\right) z_{1}+S_{y z_{2}}\left(h_{0}\right) z_{2}+S_{y z_{3}}\left(h_{0}\right) z_{3}+S_{y z_{4}}\left(h_{0}\right) z_{4}\right)
\end{array}\right) .
\end{aligned}
$$

Thus,

$$
\frac{1}{3 !} \operatorname{Proj}_{\operatorname{Im}\left(M_{3}^{1}\right)^{c}}\left(D_{y} f_{2}^{1}(z, 0,0)\left(U_{2}^{2}(z, 0)\right)\right)=\left(\begin{array}{c}
E_{2100} z_{1}^{2} z_{2}+E_{1011} z_{1} z_{3} z_{4} \\
\overline{E_{2100}} z_{1} z_{2}^{2}+\overline{E_{1011}} z_{2} z_{3} z_{4} \\
E_{0021} z_{3}^{2} z_{4}+E_{1110} z_{1} z_{2} z_{3} \\
\overline{E_{0021}} z_{3} z_{4}^{2}+\overline{E_{1110}} z_{1} z_{2} z_{4}
\end{array}\right),
$$

where

$$
\begin{aligned}
& E_{2100}=\frac{1}{6 \sqrt{l \pi}} \psi_{1}(0)\left[S_{y z_{1}}\left(h_{01100}\right)+S_{y z_{2}}\left(h_{02000}\right)\right], \\
& E_{1011}=\frac{1}{6 \sqrt{l \pi}} \psi_{1}(0)\left[S_{y z_{1}}\left(h_{00011}\right)+S_{y z_{3}}\left(h_{01001}\right)+S_{y z_{4}}\left(h_{01010}\right)\right], \\
& E_{0021}=\frac{1}{6 \sqrt{l \pi}} \psi_{3}(0)\left[S_{y z_{3}}\left(h_{00011}\right)+S_{y z_{4}}\left(h_{00020}\right)\right], \\
& E_{1110}=\frac{1}{6 \sqrt{l \pi}} \psi_{3}(0)\left[S_{y z_{1}}\left(h_{00110}\right)+S_{y z_{2}}\left(h_{01010}\right)+S_{y z_{3}}\left(h_{01100}\right)\right] .
\end{aligned}
$$

Clearly, we still need to calculate $h_{01100}, h_{02000}, h_{00011}, h_{01001}, h_{01010}, h_{00020}$, and $h_{00110}$.

From (17), (10) and (14), we have

$$
\begin{aligned}
& M_{2}^{2} U_{2}^{2}(z, 0)(\theta)=M_{2}^{2} h(z)(\theta)=D_{z} h(z) B z-A_{1}(h(z)) \\
& = \begin{cases}D_{z} h(z) B z-D_{0} \Delta h(0)-L_{0}(h(z)), & \theta=0 \\
D_{z} h(z) B z-D_{\theta} h(z), & \theta \neq 0, \\
\sum_{j \geq 0}\left[D_{z} h_{j}(z) \gamma_{j}(x) B z-D_{0} \Delta h_{j}(0) \gamma_{j}(x)-L_{0}\left(h_{j}(z) \gamma_{j}(x)\right)\right], & \theta=0 \\
\sum_{j \geq 0}\left[D_{z} h_{j}(z) \gamma_{j}(x) B z-D_{\theta} h_{j}(z) \gamma_{j}(x)\right], & \theta \neq 0 .\end{cases}
\end{aligned}
$$


According to (16),

$$
\begin{aligned}
& f_{2}^{2}(z, 0,0) \\
& = \begin{cases}\widetilde{F}_{2}(z, 0,0)-\phi_{1}(0) f_{2}^{1(1)}(z, 0,0) \gamma_{n_{1}}-\phi_{2}(0) f_{2}^{1(2)}(z, 0,0) \gamma_{n_{1}} & \theta=0, \\
-\phi_{3}(0) f_{2}^{1(3)}(z, 0,0) \gamma_{n_{2}}-\phi_{4}(0) f_{2}^{1(4)}(z, 0,0) \gamma_{n_{2}}, & \\
-\phi_{1}(\theta) f_{2}^{1(1)}(z, 0,0) \gamma_{n_{1}}-\phi_{2}(\theta) f_{2}^{1(2)}(z, 0,0) \gamma_{n_{1}} & \theta \neq 0 . \\
-\phi_{3}(\theta) f_{2}^{1(3)}(z, 0,0) \gamma_{n_{2}}-\phi_{4}(\theta) f_{2}^{1(4)}(z, 0,0) \gamma_{n_{2},} & \end{cases}
\end{aligned}
$$

From (16), (18), and (19), we have

$$
\left\langle M_{2}^{2}\left(U_{2}^{2}(z, 0)\right), \beta_{j}\right\rangle=\left\langle f_{2}^{2}(z, 0,0), \beta_{j}\right\rangle
$$

Matching the coefficients of $z_{1}^{q_{1}} z_{2}^{q_{2}} z_{3}^{q_{3}} z_{4}^{q_{4}}$ in (47) when $j=0$, we can get the results of $h_{01100}$, $h_{02000}, h_{00011}, h_{01001}, h_{01010}, h_{00020}$, and $h_{00110}$. We take $h_{02000}(\theta)$ as an example, and the others can be calculated in the same method.

When $\theta \neq 0$, solve the following equation

$$
\begin{aligned}
2 i \omega_{1} h_{02000}(\theta)-\dot{h}_{02000}(\theta)= & -\left\langle\phi_{1}(\theta) f_{2}^{1(1)} \gamma_{n_{1}}, \beta_{0}\right\rangle-\left\langle\phi_{2}(\theta) f_{2}^{1(2)} \gamma_{n_{1}}, \beta_{0}\right\rangle \\
& -\left\langle\phi_{3}(\theta) f_{2}^{1(3)} \gamma_{n_{2}}, \beta_{0}\right\rangle-\left\langle\phi_{4}(\theta) f_{2}^{1(4)} \gamma_{n_{2}}, \beta_{0}\right\rangle,
\end{aligned}
$$

and we get

$$
\begin{aligned}
h_{02000}(\theta) & =e^{2 i \omega_{1} \theta} h_{02000}(0)+\frac{1}{-i \omega_{1}}\left(e^{i \omega_{1} \theta}-e^{2 i \omega_{1} \theta}\right) \phi_{1}(0) f_{2000}^{1(1)} \\
& +\frac{1}{-3 i \omega_{1}}\left(e^{-i \omega_{1} \theta}-e^{2 i \omega_{1} \theta}\right) \phi_{2}(0) f_{2000}^{1(2)}+\frac{1}{-2 i \omega_{1}+i \omega_{2}}\left(e^{i \omega_{2} \theta}-e^{2 i \omega_{1} \theta}\right) \phi_{3}(0) f_{2000}^{1(3)} \\
& +\frac{1}{-2 i \omega_{1}-i \omega_{2}}\left(e^{-i \omega_{2} \theta}-e^{2 i \omega_{1} \theta}\right) \phi_{4}(0) f_{2000}^{1(4)} .
\end{aligned}
$$

When $\theta=0$,

$$
\begin{array}{r}
2 i \omega_{1} h_{02000}(0)-D_{0} \Delta h_{02000}(0)-L_{0}\left(h_{02000}\right)=\left\langle F_{2000} \gamma_{n_{1}}^{2}, \beta_{0}\right\rangle-\phi_{1}(0) f_{2}^{1(1)}\left\langle\gamma_{n_{1}}, \beta_{0}\right\rangle \\
-\phi_{2}(0) f_{2}^{1(2)}\left\langle\gamma_{n_{1}}, \beta_{0}\right\rangle-\phi_{3}(0) f_{2}^{1(3)}\left\langle\gamma_{n_{2}}, \beta_{0}\right\rangle-\phi_{4}(0) f_{2}^{1(4)}\left\langle\gamma_{n_{2}}, \beta_{0}\right\rangle .
\end{array}
$$

Combining with

$$
\begin{aligned}
& {\left[i \omega_{1}-\frac{n_{1}^{2}}{l^{2}} D_{0}-L_{0}\left(e^{i \omega_{1} \cdot} I_{d}\right)\right] \phi_{1}(0)=0, \quad\left[-i \omega_{1}-\frac{n_{1}^{2}}{l^{2}} D_{0}-L_{0}\left(e^{-i \omega_{1} \cdot} I_{d}\right)\right] \phi_{2}(0)=0,} \\
& {\left[i \omega_{2}-\frac{n_{2}^{2}}{l^{2}} D_{0}-L_{0}\left(e^{i \omega_{2} \cdot} I_{d}\right)\right] \phi_{3}(0)=0, \quad\left[-i \omega_{2}-\frac{n_{2}^{2}}{l^{2}} D_{0}-L_{0}\left(e^{-i \omega_{2} \cdot} I_{d}\right)\right] \phi_{4}(0)=0,}
\end{aligned}
$$


we can get

$$
\begin{aligned}
h_{02000}(0) & =\frac{1}{-i \omega_{1}} \phi_{1}(0) f_{2000}^{1(1)}+\frac{1}{-3 i \omega_{1}} \phi_{2}(0) f_{2000}^{1(2)}+\frac{1}{-2 i \omega_{1}+i \omega_{2}} \phi_{3}(0) f_{2000}^{1(3)} \\
& +\frac{1}{-2 i \omega_{1}-i \omega_{2}} \phi_{4}(0) f_{2000}^{1(4)}-\left[-2 i \omega_{1}+L_{0}\left(e^{2 i \omega_{1} \cdot} I_{d}\right)\right]^{-1}\left\langle F_{2000} \gamma_{n_{1}}^{2}, \beta_{0}\right\rangle,
\end{aligned}
$$

and

$$
\begin{aligned}
h_{02000}(\theta) & =\frac{1}{-i \omega_{1}} \phi_{1}(\theta) f_{2000}^{1(1)}+\frac{1}{-3 i \omega_{1}} \phi_{2}(\theta) f_{2000}^{1(2)}+\frac{1}{-2 i \omega_{1}+i \omega_{2}} \phi_{3}(\theta) f_{2000}^{1(3)} \\
& +\frac{1}{-2 i \omega_{1}-i \omega_{2}} \phi_{4}(\theta) f_{2000}^{1(4)}-\frac{1}{\sqrt{l \pi}} e^{2 i \omega_{1} \theta}\left[-2 i \omega_{1}+L_{0}\left(e^{2 i \omega_{1}} I_{d}\right)\right]^{-1} F_{2000} .
\end{aligned}
$$

Using the same method mentioned above, we can work out

$$
\begin{aligned}
& h_{01100}(\theta)=\frac{1}{i \omega_{1}} \phi_{1}(\theta) f_{1100}^{1(1)}+\frac{1}{-i \omega_{1}} \phi_{2}(\theta) f_{1100}^{1(2)} \\
& +\frac{1}{i \omega_{2}} \phi_{3}(\theta) f_{1100}^{1(3)}+\frac{1}{-i \omega_{2}} \phi_{4}(\theta) f_{1100}^{1(4)}-\frac{1}{\sqrt{l \pi}}\left[L_{0}\left(I_{d}\right)\right]^{-1} F_{1100}, \\
& h_{00011}(\theta)=\frac{1}{i \omega_{1}} \phi_{1}(\theta) f_{0011}^{1(1)}+\frac{1}{-i \omega_{1}} \phi_{2}(\theta) f_{0011}^{1(2)} \\
& +\frac{1}{i \omega_{2}} \phi_{3}(\theta) f_{0011}^{1(3)}+\frac{1}{-i \omega_{2}} \phi_{4}(\theta) f_{0011}^{1(4)}-\frac{1}{\sqrt{l \pi}}\left[L_{0}\left(I_{d}\right)\right]^{-1} F_{0011}, \\
& h_{01001}(\theta)=\frac{1}{i \omega_{2}} \phi_{1}(\theta) f_{1001}^{1(1)}+\frac{1}{-2 i \omega_{1}+i \omega_{2}} \phi_{2}(\theta) f_{1001}^{1(2)} \\
& +\frac{1}{-i \omega_{1}+2 i \omega_{2}} \phi_{3}(\theta) f_{1001}^{1(3)}+\frac{1}{-i \omega_{1}} \phi_{4}(\theta) f_{1001}^{1(4)} \\
& -\frac{1}{\sqrt{l \pi}} e^{\left(i \omega_{1}-i \omega_{2}\right) \theta}\left[-\left(i \omega_{1}-i \omega_{2}\right)+L_{0}\left(e^{\left(i \omega_{1}-i \omega_{2}\right)} \cdot I_{d}\right)\right]^{-1} F_{1001} \text {, } \\
& h_{01010}(\theta)=\frac{1}{-i \omega_{2}} \phi_{1}(\theta) f_{1010}^{1(1)}+\frac{1}{-2 i \omega_{1}-i \omega_{2}} \phi_{2}(\theta) f_{1010}^{1(2)} \\
& +\frac{1}{-i \omega_{1}} \phi_{3}(\theta) f_{1010}^{1(3)}+\frac{1}{-i \omega_{1}-2 i \omega_{2}} \phi_{4}(\theta) f_{1010}^{1(4)} \\
& -\frac{1}{\sqrt{l \pi}} e^{\left(i \omega_{1}+i \omega_{2}\right) \theta}\left[-\left(i \omega_{1}+i \omega_{2}\right)+L_{0}\left(e^{\left(i \omega_{1}+i \omega_{2}\right)} \cdot I_{d}\right)\right]^{-1} F_{1010} \\
& h_{00110}(\theta)=\frac{1}{2 i \omega_{1}-i \omega_{2}} \phi_{1}(\theta) f_{0110}^{1(1)}+\frac{1}{-i \omega_{2}} \phi_{2}(\theta) f_{0110}^{1(2)} \\
& +\frac{1}{i \omega_{1}} \phi_{3}(\theta) f_{0110}^{1(3)}+\frac{1}{i \omega_{1}-2 i \omega_{2}} \phi_{4}(\theta) f_{0110}^{1(4)} \\
& -\frac{1}{\sqrt{l \pi}} e^{\left(-i \omega_{1}+i \omega_{2}\right) \theta}\left[-\left(-i \omega_{1}+i \omega_{2}\right)+L_{0}\left(e^{\left(-i \omega_{1}+i \omega_{2}\right)} \cdot I_{d}\right)\right]^{-1} F_{0110}
\end{aligned}
$$

and

$$
\begin{aligned}
h_{00020}(\theta) & =\frac{1}{i \omega_{1}-2 i \omega_{2}} \phi_{1}(\theta) f_{0020}^{1(1)}+\frac{1}{-i \omega_{1}-2 i \omega_{2}} \phi_{2}(\theta) f_{0020}^{1(2)}+\frac{1}{-i \omega_{2}} \phi_{3}(\theta) f_{0020}^{1(3)} \\
& +\frac{1}{-3 i \omega_{2}} \phi_{4}(\theta) f_{0020}^{1(4)}-\frac{1}{\sqrt{l \pi}} e^{2 i \omega_{2} \theta}\left[-2 i \omega_{2}+L_{0}\left(e^{2 i \omega_{2} \cdot} I_{d}\right)\right]^{-1} F_{0020} .
\end{aligned}
$$


Case 2 When $n_{1}=0, n_{2} \neq 0$, we have

$$
\begin{aligned}
& b_{j n_{1} n_{1}}=\int_{0}^{l \pi} \gamma_{j}(x) \gamma_{0}(x) \gamma_{0}(x) d x=\left\{\begin{array}{l}
\frac{1}{\sqrt{l \pi}}, j=0, \\
0, \quad j \neq 0,
\end{array}\right. \\
& b_{j n_{1} n_{2}}=b_{j n_{2} n_{1}}=\int_{0}^{l \pi} \gamma_{j}(x) \gamma_{0}(x) \gamma_{n_{2}}(x) d x= \begin{cases}\frac{1}{\sqrt{l \pi}}, & j=n_{2}, \\
0, & j \neq n_{2},\end{cases} \\
& b_{j n_{2} n_{2}}=\int_{0}^{l \pi} \gamma_{j}(x) \gamma_{n_{2}}(x) \gamma_{n_{2}}(x) d x= \begin{cases}\frac{1}{\sqrt{l \pi}}, & j=0, \\
\frac{1}{\sqrt{2 l \pi}}, & j=2 n_{2}, \\
0, & \text { otherwise, }\end{cases}
\end{aligned}
$$

and

$$
\begin{aligned}
& D_{y} f_{2}^{1}(z, 0,0)\left(U_{2}^{2}(z, 0)\right) \\
& =\Psi(0)\left(\begin{array}{c}
\sum_{j \geq 0}\left[b_{j n_{1} n_{1}}\left(S_{y z_{1}}\left(h_{j}\right) z_{1}+S_{y z_{2}}\left(h_{j}\right) z_{2}\right)+b_{j n_{2} n_{1}}\left(S_{y z_{3}}\left(h_{j}\right) z_{3}+S_{y z_{4}}\left(h_{j}\right) z_{4}\right)\right] \\
\sum_{j \geq 0}\left[b_{j n_{1} n_{2}}\left(S_{y z_{1}}\left(h_{j}\right) z_{1}+S_{y z_{2}}\left(h_{j}\right) z_{2}\right)+b_{j n_{2} n_{2}}\left(S_{y z_{3}}\left(h_{j}\right) z_{3}+S_{y z_{4}}\left(h_{j}\right) z_{4}\right)\right]
\end{array}\right), \\
& =\Psi(0)\left(\begin{array}{c}
\frac{1}{\sqrt{l \pi}}\left(S_{y z_{1}}\left(h_{0}\right) z_{1}+S_{y z_{2}}\left(h_{0}\right) z_{2}\right)+\frac{1}{\sqrt{l \pi}}\left(S_{y z_{3}}\left(h_{n_{2}}\right) z_{3}+S_{y z_{4}}\left(h_{n_{2}}\right) z_{4}\right) \\
\frac{1}{\sqrt{l \pi}}\left(S_{y z_{1}}\left(h_{n_{2}}\right) z_{1}+S_{y z_{2}}\left(h_{n_{2}}\right) z_{2}\right)+\frac{1}{\sqrt{l \pi}}\left(S_{y z_{3}}\left(h_{0}\right) z_{3}+S_{y z_{4}}\left(h_{0}\right) z_{4}\right) \\
+\frac{1}{\sqrt{2 l \pi}}\left(S_{y z_{3}}\left(h_{2 n_{2}}\right) z_{3}+S_{y z_{4}}\left(h_{2 n_{2}}\right) z_{4}\right)
\end{array}\right) .
\end{aligned}
$$

Thus, we obtain

$$
\frac{1}{3 !} \operatorname{Proj}_{\operatorname{Ker}\left(M_{3}^{1}\right)^{c}}\left(D_{y} f_{2}^{1}(z, 0,0)\left(U_{2}^{2}(z, 0)\right)\right)=\left(\begin{array}{l}
E_{2100} z_{1}^{2} z_{2}+E_{1011} z_{1} z_{3} z_{4} \\
\overline{E_{2100}} z_{1} z_{2}^{2}+\overline{E_{1011}} z_{2} z_{3} z_{4} \\
E_{0021} z_{3}^{2} z_{4}+E_{1110} z_{1} z_{2} z_{3} \\
\overline{E_{0021}} z_{3} z_{4}^{2}+\overline{E_{1110}} z_{1} z_{2} z_{4}
\end{array}\right)
$$

where

$$
\begin{aligned}
E_{2100} & =\frac{1}{6 \sqrt{l \pi}} \psi_{1}(0)\left[S_{y z_{1}}\left(h_{01100}\right)+S_{y z_{2}}\left(h_{02000}\right)\right], \\
E_{1011} & \left.=\frac{1}{6 \sqrt{l \pi}} \psi_{1}(0)\left[S_{y z_{1}} h_{00011}\right)+S_{y z_{3}}\left(h_{n_{2} 1001}\right)+S_{y z_{4}}\left(h_{n_{2} 1010}\right)\right], \\
E_{0021} & =\frac{1}{6} \psi_{3}(0)\left[\frac{1}{\sqrt{l \pi}}\left(S_{y z_{3}}\left(h_{00011}\right)+S_{y z_{4}}\left(h_{00020}\right)\right)+\frac{1}{\sqrt{2 l \pi}}\left(S_{y z_{3}}\left(h_{2 n_{2} 0011}\right)+S_{y z_{4}}\left(h_{2 n_{2} 0020}\right)\right)\right], \\
E_{1110} & =\frac{1}{6} \psi_{3}(0)\left[\frac{1}{\sqrt{l \pi}}\left(S_{y z_{1}}\left(h_{n_{2} 0110}\right)+S_{y z_{2}}\left(h_{n_{2} 1010}\right)+S_{y z_{3}}\left(h_{01100}\right)\right)+\frac{1}{\sqrt{2 l \pi}} S_{y z_{3}}\left(h_{2 n_{2} 1100}\right)\right] .
\end{aligned}
$$

Clearly, we still need to calculate $h_{01100}, h_{02000}, h_{00011}, h_{n_{2} 1001}, h_{n_{2} 1010}, h_{00020}, h_{2 n_{2} 0011}$, $h_{2 n_{2} 0020}, h_{n_{2} 0110}$, and $h_{2 n_{2} 1100}$. Using the same method used in case 1 , we can get the 
following results

$$
\begin{aligned}
& h_{02000}(\theta)=\frac{1}{-i \omega_{1}} \phi_{1}(\theta) f_{2000}^{1(1)}+\frac{1}{-3 i \omega_{1}} \phi_{2}(\theta) f_{2000}^{1(2)} \\
& -\frac{1}{\sqrt{l \pi}} e^{2 i \omega_{1} \theta}\left[-2 i \omega_{1}+L_{0}\left(e^{2 i \omega_{1} \cdot} I_{d}\right)\right]^{-1} F_{2000}, \\
& h_{01100}(\theta)=\frac{1}{i \omega_{1}} \phi_{1}(\theta) f_{1100}^{1(1)}+\frac{1}{-i \omega_{1}} \phi_{2}(\theta) f_{1100}^{1(2)}-\frac{1}{\sqrt{l \pi}}\left[L_{0}\left(I_{d}\right)\right]^{-1} F_{1100}, \\
& h_{00011}(\theta)=\frac{1}{i \omega_{1}} \phi_{1}(\theta) f_{0011}^{1(1)}+\frac{1}{-i \omega_{1}} \phi_{2}(\theta) f_{0011}^{1(2)}-\frac{1}{\sqrt{l \pi}}\left[L_{0}\left(I_{d}\right)\right]^{-1} F_{0011}, \\
& h_{n_{2} 1001}(\theta)=\frac{1}{-i \omega_{1}+2 i \omega_{2}} \phi_{3}(\theta) f_{1001}^{1(3)}+\frac{1}{-i \omega_{1}} \phi_{4}(\theta) f_{1001}^{1(4)} \\
& -\frac{1}{\sqrt{l \pi}} e^{\left(i \omega_{1}-i \omega_{2}\right) \theta}\left[-\left(i \omega_{1}-i \omega_{2}\right)-\frac{n_{2}^{2}}{l^{2}} D_{0}+L_{0}\left(e^{\left(i \omega_{1}-i \omega_{2}\right)} \cdot I_{d}\right)\right]^{-1} F_{1001} \\
& h_{n_{2} 1010}(\theta)=\frac{1}{-i \omega_{1}} \phi_{3}(\theta) f_{1010}^{1(3)}+\frac{1}{-i \omega_{1}-2 i \omega_{2}} \phi_{4}(\theta) f_{1010}^{1(4)} \\
& -\frac{1}{\sqrt{l \pi}} e^{\left(i \omega_{1}+i \omega_{2}\right) \theta}\left[-\left(i \omega_{1}+i \omega_{2}\right)-\frac{n_{2}^{2}}{l^{2}} D_{0}+L_{0}\left(e^{\left(i \omega_{1}+i \omega_{2}\right)} \cdot I_{d}\right)\right]^{-1} F_{1010} \\
& h_{00020}(\theta)=\frac{1}{i \omega_{1}-2 i \omega_{2}} \phi_{1}(\theta) f_{0020}^{1(1)}+\frac{1}{-i \omega_{1}-2 i \omega_{2}} \phi_{2}(\theta) f_{0020}^{1(2)} \\
& -\frac{1}{\sqrt{l \pi}} e^{2 i \omega_{2} \theta}\left[-2 i \omega_{2}+L_{0}\left(e^{2 i \omega_{2} \cdot} I_{d}\right)\right]^{-1} F_{0002}, \\
& h_{n_{2} 0110}(\theta)=\frac{1}{i \omega_{1}} \phi_{3}(\theta) f_{0110}^{1(3)}+\frac{1}{i \omega_{1}-2 i \omega_{2}} \phi_{4}(\theta) f_{0110}^{1(4)} \\
& -\frac{1}{\sqrt{l \pi}} e^{\left(-i \omega_{1}+i \omega_{2}\right) \theta}\left[-\left(-i \omega_{1}+i \omega_{2}\right)-\frac{n_{2}^{2}}{l^{2}} D_{0}+L_{0}\left(e^{\left(-i \omega_{1}+i \omega_{2}\right)} \cdot I_{d}\right)\right]^{-1} F_{0110}, \\
& h_{2 n_{2} 0011}(\theta)=-\frac{1}{\sqrt{2 l \pi}}\left[-\frac{\left(2 n_{2}\right)^{2}}{l^{2}} D_{0}+L_{0}\left(I_{d}\right)\right]^{-1} F_{0011} \\
& h_{2 n_{2} 0020}(\theta)=-\frac{1}{\sqrt{2 l \pi}} e^{2 i \omega_{2} \theta}\left[-2 i \omega_{2}-\frac{\left(2 n_{2}\right)^{2}}{l^{2}} D_{0}+L_{0}\left(e^{2 i \omega_{2} \cdot} I_{d}\right)\right]^{-1} F_{0020},
\end{aligned}
$$

and

$$
h_{2 n_{2} 1100}(\theta)=0 \text {. }
$$


Case 3 When $n_{1} \neq 0, n_{2} \neq 0$, from

$$
\begin{aligned}
& b_{j n_{k} n_{k}}=\int_{0}^{l \pi} \gamma_{j}(x) \gamma_{n_{k}}(x) \gamma_{n_{k}}(x) d x=\left\{\begin{array}{l}
\frac{1}{\sqrt{l \pi}}, \quad j=0, \\
\frac{1}{\sqrt{2 l \pi}}, j=2 n_{k}, \quad(k=1,2), \\
0, \quad \text { otherwise, }
\end{array}\right. \\
& b_{j n_{1} n_{2}}=b_{j n_{2} n_{1}}=\int_{0}^{l \pi} \gamma_{j}(x) \gamma_{n_{1}}(x) \gamma_{n_{2}}(x) d x= \begin{cases}\frac{1}{\sqrt{2 l \pi}}, & j=n_{1}+n_{2}, \\
\frac{1}{\sqrt{2 l \pi}}, & j=n_{2}-n_{1} \neq 0, \\
\frac{1}{\sqrt{l \pi}}, & j=n_{2}-n_{1}=0, \\
0, & \text { otherwise, }\end{cases}
\end{aligned}
$$

we have

$$
\begin{aligned}
& D_{y} f_{2}^{1}(z, 0,0)\left(U_{2}^{2}(z, 0)\right) \\
& =\Psi(0)\left(\begin{array}{c}
\sum_{j \geq 0}\left[b_{j n_{1} n_{1}}\left(S_{y z_{1}}\left(h_{j}\right) z_{1}+S_{y z_{2}}\left(h_{j}\right) z_{2}\right)+b_{j n_{2} n_{1}}\left(S_{y z_{3}}\left(h_{j}\right) z_{3}+S_{y z_{4}}\left(h_{j}\right) z_{4}\right)\right] \\
\sum_{j \geq 0}\left[b_{j n_{1} n_{2}}\left(S_{y z_{1}}\left(h_{j}\right) z_{1}+S_{y z_{2}}\left(h_{j}\right) z_{2}\right)+b_{j n_{2} n_{2}}\left(S_{y z_{3}}\left(h_{j}\right) z_{3}+S_{y z_{4}}\left(h_{j}\right) z_{4}\right)\right]
\end{array}\right) \\
& =\Psi(0)\left(\begin{array}{c}
b_{0 n_{1} n_{1}}\left(S_{y z_{1}}\left(h_{0}\right) z_{1}+S_{y z_{2}}\left(h_{0}\right) z_{2}\right)+b_{2 n_{1} n_{1} n_{1}}\left(S_{y z_{1}}\left(h_{2 n_{1}}\right) z_{1}+S_{y z_{2}}\left(h_{2 n_{1}}\right) z_{2}\right) \\
+b_{\left(n_{1}+n_{2}\right) n_{2} n_{1}}\left(S_{y z_{3}}\left(h_{n_{1}+n_{2}}\right) z_{3}+S_{y z_{4}}\left(h_{n_{1}+n_{2}}\right) z_{4}\right) \\
+b_{n_{2}-n_{1} n_{2} n_{1}}\left(S_{y z_{3}}\left(h_{n_{2}-n_{1}}\right) z_{3}+S_{y z_{4}}\left(h_{n_{2}-n_{1}}\right) z_{4}\right) \\
b_{\left(n_{1}+n_{2}\right) n_{1} n_{2}}\left(S_{y z_{1}}\left(h_{n_{1}+n_{2}}\right) z_{1}+S_{y z_{2}}\left(h_{n_{1}+n_{2}}\right) z_{2}\right) \\
+b_{\left(n_{2}-n_{1}\right) n_{1} n_{2}}\left(S_{y z_{1}}\left(h_{n_{2}-n_{1}}\right) z_{1}+S_{y z_{2}}\left(h_{n_{2}-n_{1}}\right) z_{2}\right) \\
+b_{0 n_{2} n_{2}}\left(S_{y z_{3}}\left(h_{0}\right) z_{3}+S_{y z_{4}}\left(h_{0}\right) z_{4}\right)+b_{2 n_{2} n_{2} n_{2}}\left(S_{y z_{3}}\left(h_{2 n_{2}}\right) z_{3}+S_{y z_{4}}\left(h_{2 n_{2}}\right) z_{4}\right)
\end{array}\right) \\
& =\Psi(0)\left(\begin{array}{c}
\frac{1}{\sqrt{l \pi}}\left(S_{y z_{1}}\left(h_{0}\right) z_{1}+S_{y z_{2}}\left(h_{0}\right) z_{2}\right)+\frac{1}{\sqrt{2 l \pi}}\left(S_{y z_{1}}\left(h_{2 n_{1}}\right) z_{1}+S_{y z_{2}}\left(h_{2 n_{1}}\right) z_{2}\right) \\
+\frac{1}{\sqrt{2 l \pi}}\left(S_{y z_{3}}\left(h_{n_{1}+n_{2}}\right) z_{3}+S_{y z_{4}}\left(h_{n_{1}+n_{2}}\right) z_{4}\right) \\
+b_{n_{2}-n_{1} n_{2} n_{1}}\left(S_{y z_{3}}\left(h_{n_{2}-n_{1}}\right) z_{3}+S_{y z_{4}}\left(h_{n_{2}-n_{1}}\right) z_{4}\right) \\
\frac{1}{\sqrt{2 l \pi}}\left(S_{y z_{1}}\left(h_{n_{1}+n_{2}}\right) z_{1}+S_{y z_{2}}\left(h_{n_{1}+n_{2}}\right) z_{2}\right) \\
+b_{\left(n_{2}-n_{1}\right) n_{1} n_{2}}\left(S_{y z_{1}}\left(h_{n_{2}-n_{1}}\right) z_{1}+S_{y z_{2}}\left(h_{n_{2}-n_{1}}\right) z_{2}\right) \\
+\frac{1}{\sqrt{l \pi}}\left(S_{y z_{3}}\left(h_{0}\right) z_{3}+S_{y z_{4}}\left(h_{0}\right) z_{4}\right)+\frac{1}{\sqrt{2 l \pi}}\left(S_{y z_{3}}\left(h_{2 n_{2}}\right) z_{3}+S_{y z_{4}}\left(h_{2 n_{2}}\right) z_{4}\right)
\end{array}\right),
\end{aligned}
$$

thus,

$$
\operatorname{Proj}_{\operatorname{Im}\left(M_{3}^{1}\right)^{c}} D_{y} f_{2}^{1}(z, 0,0)\left(U_{2}^{2}(z, 0)\right)=\left(\begin{array}{l}
E_{2100} z_{1}^{2} z_{2}+E_{1011} z_{1} z_{3} z_{4} \\
\overline{E_{2100}} z_{1} z_{2}^{2}+\overline{E_{1011}} z_{2} z_{3} z_{4} \\
E_{0021} z_{3}^{2} z_{4}+E_{1110} z_{1} z_{2} z_{3} \\
\overline{E_{0021}} z_{3} z_{4}^{2}+\overline{E_{1110}} z_{1} z_{2} z_{4}
\end{array}\right)
$$


where

$$
\begin{aligned}
E_{2100}= & \frac{1}{6} \psi_{1}(0)\left[\frac{1}{\sqrt{l \pi}}\left(S_{y z_{1}}\left(h_{01100}\right)+S_{y z_{2}}\left(h_{02000}\right)\right)+\frac{1}{\sqrt{2 l \pi}}\left(S_{y z_{1}}\left(h_{2 n_{1} 1100}\right)+S_{y z_{2}}\left(h_{2 n_{1} 2000}\right)\right)\right], \\
E_{1011}= & \frac{1}{6} \psi_{1}(0)\left[\frac { 1 } { \sqrt { l \pi } } \left(S_{y z_{1}}\left(h_{00011}\right)+\frac{1}{\sqrt{2 l \pi}}\left(S_{y z_{1}}\left(h_{2 n_{1} 0011}\right)+S_{y z_{3}}\left(h_{\left(n_{1}+n_{2}\right) 1001}\right)\right.\right.\right. \\
& \left.\left.+S_{y z_{4}}\left(h_{\left(n_{1}+n_{2}\right) 1010}\right)\right)+b_{\left(n_{2}-n_{1}\right) n_{2} n_{1}}\left(S_{y z_{3}}\left(h_{\left(n_{2}-n_{1}\right) 1001}\right)+S_{y z_{4}}\left(h_{\left(n_{2}-n_{1}\right) 1010}\right)\right)\right], \\
E_{0021}= & \frac{1}{6} \psi_{3}(0)\left[\frac{1}{\sqrt{l \pi}}\left(S_{y z_{3}}\left(h_{00011}\right)+S_{y z_{4}}\left(h_{00020}\right)\right)+\frac{1}{\sqrt{2 l \pi}}\left(S_{y z_{3}}\left(h_{2 n_{2} 0011}\right)+S_{y z_{4}}\left(h_{2 n_{2} 0020}\right)\right)\right], \\
E_{1110}= & \frac{1}{6} \psi_{3}(0)\left[\frac { 1 } { \sqrt { l \pi } } \left(S_{y z_{3}}\left(h_{01100}\right)+\frac{1}{\sqrt{2 l \pi}}\left(S_{y z_{3}}\left(h_{2 n_{2} 1100}\right)+S_{y z_{1}}\left(h_{\left(n_{1}+n_{2}\right) 0110}\right)\right.\right.\right. \\
& \left.\left.+S_{y z_{2}}\left(h_{\left(n_{1}+n_{2}\right) 1010}\right)\right)+b_{\left(n_{2}-n_{1}\right) n_{1} n_{2}}\left(S_{y z_{1}}\left(h_{\left(n_{2}-n_{1}\right) 0110}\right)+S_{y z_{2}}\left(h_{\left(n_{2}-n_{1}\right) 1010}\right)\right)\right] .
\end{aligned}
$$

Clearly, we still need to calculate $h_{01100}, h_{02000}, h_{2 n_{1} 1100}, h_{2 n_{1} 2000}, h_{00011}, h_{2 n_{1} 0011}, h_{\left(n_{1}+n_{2}\right) 1001}$, $h_{\left(n_{1}+n_{2}\right) 1010}, h_{\left(n_{2}-n_{1}\right) 1001}, h_{\left(n_{2}-n_{1}\right) 1010}, h_{00020}, h_{2 n_{2} 0011}, h_{2 n_{2} 0020}, h_{2 n_{2} 1100}, h_{\left(n_{1}+n_{2}\right) 0110}, h_{\left(n_{1}+n_{2}\right) 1010}$, $h_{\left(n_{2}-n_{1}\right) 0110}$, and $h_{\left(n_{2}-n_{1}\right) 1010}$. In fact, we have

$$
\begin{aligned}
& h_{02000}(\theta)=-\frac{1}{\sqrt{l \pi}} e^{2 i \omega_{1} \theta}\left[-2 i \omega_{1}+L_{0}\left(e^{2 i \omega_{1} \cdot} I_{d}\right)\right]^{-1} F_{2000}, \\
& h_{01100}(\theta)=-\frac{1}{\sqrt{l \pi}}\left[L_{0}\left(I_{d}\right)\right]^{-1} F_{1100}, \\
& h_{2 n_{1} 2000}(\theta)= \begin{cases}-\frac{1}{\sqrt{2 l \pi}} e^{2 i \omega_{1} \theta}\left[-2 i \omega_{1}-\frac{\left(2 n_{1}\right)^{2}}{l^{2}} D_{0}+L_{0}\left(e^{2 i \omega_{1} \cdot} I_{d}\right)\right]^{-1} F_{2000}, & n_{2} \neq 2 n_{1}, \\
\frac{1}{-2 i \omega_{1}+i \omega_{2}} \phi_{3}(\theta) f_{2000}^{1(3)}+\frac{1}{-2 i \omega_{1}-i \omega_{2}} \phi_{4}(\theta) f_{2000}^{1(4)} & \\
-\frac{1}{\sqrt{2 l \pi}}\left[-2 i \omega_{1}-\frac{\left(2 n_{1}\right)^{2}}{l^{2}} D_{0}+L_{0}\left(e^{2 i \omega_{1}} I_{d}\right)\right]^{-1} F_{2000}, & n_{2}=2 n_{1},\end{cases} \\
& h_{2 n_{1} 1100}(\theta)= \begin{cases}-\frac{1}{\sqrt{2 l \pi}}\left[-D_{0} \frac{\left(2 n_{1}\right)^{2}}{l^{2}}+L_{0}\left(I_{d}\right)\right]^{-1} F_{1100}, & n_{2} \neq 2 n_{1}, \\
-\frac{1}{\sqrt{2 l \pi}}\left[-D_{0} \frac{\left(2 n_{1}\right)^{2}}{l^{2}}+L_{0}\left(I_{d}\right)\right]^{-1} F_{1100}+\frac{1}{i \omega_{2}} \phi_{3}(\theta) f_{1100}^{1(3)} & \\
+\frac{1}{-i \omega_{2}} \phi_{4}(\theta) f_{1100}^{1(4)}, & n_{2}=2 n_{1},\end{cases} \\
& h_{00011}(\theta)=-\frac{1}{\sqrt{l \pi}}\left[L_{0}\left(I_{d}\right)\right]^{-1} F_{0011}, \\
& h_{2 n_{1} 0011}(\theta)= \begin{cases}-\frac{1}{\sqrt{2 l \pi}}\left[-D_{0} \frac{\left(2 n_{1}\right)^{2}}{l^{2}}+L_{0}\left(I_{d}\right)\right]^{-1} F_{0011}, & n_{2}=n_{1}, \\
\frac{1}{i \omega_{2}} \phi_{3}(\theta) f_{0011}^{1,3}+\frac{1}{-i \omega_{2}} \phi_{4}(\theta) f_{0011}^{1,4}, & n_{2}=2 n_{1}, \\
0, & \text { otherwise }\end{cases} \\
& h_{n_{1}+n_{2} 1001}(\theta)=-\frac{1}{\sqrt{2 l \pi}} e^{\left(i \omega_{1}-i \omega_{2}\right) \theta}\left[-\left(i \omega_{1}-i \omega_{2}\right)\right. \\
& \left.-\frac{\left(n_{1}+n_{2}\right)^{2}}{l^{2}} D_{0}+L_{0}\left(e^{\left(i \omega_{1}-i \omega_{2}\right)} \cdot I_{d}\right)\right]^{-1} F_{1001},
\end{aligned}
$$




$$
\begin{aligned}
& h_{n_{1}+n_{2} 1010}(\theta)=-\frac{1}{\sqrt{2 l \pi}} e^{\left(i \omega_{1}+i \omega_{2}\right) \theta}\left[-\left(i \omega_{1}+i \omega_{2}\right)\right. \\
& \left.-\frac{\left(n_{1}+n_{2}\right)^{2}}{l^{2}} D_{0}+L_{0}\left(e^{\left(i \omega_{1}+i \omega_{2}\right)} \cdot I_{d}\right)\right]^{-1} F_{1010},
\end{aligned}
$$

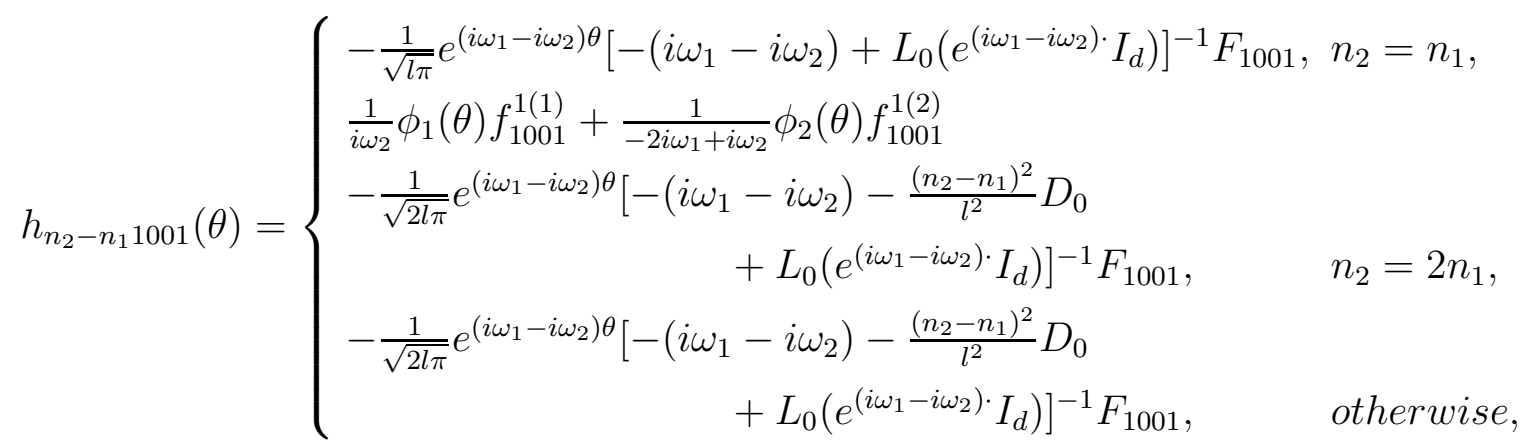

$$
\begin{aligned}
& h_{n_{2}-n_{1} 1010}(\theta)=\left\{\begin{array}{cc}
-\frac{1}{\sqrt{l \pi}} e^{\left(i \omega_{1}+i \omega_{2}\right) \theta}\left[-\left(i \omega_{1}+i \omega_{2}\right)+L_{0}\left(e^{\left(i \omega_{1}+i \omega_{2}\right) \cdot} I_{d}\right)\right]^{-1} F_{1010}, & n_{2}=n_{1}, \\
\frac{1}{-i \omega_{2}} \phi_{1}(\theta) f_{1010}^{1(1)}+\frac{1}{-2 i \omega_{1}-i \omega_{2}} \phi_{2}(\theta) f_{1010}^{1(2)} & \\
-\frac{1}{\sqrt{2 l \pi}} e^{\left(i \omega_{1}+i \omega_{2}\right) \theta}\left[-\left(i \omega_{1}+i \omega_{2}\right)-\frac{\left(n_{2}-n_{1}\right)^{2}}{l^{2}} D_{0}\right. & n_{2}=2 n_{1}, \\
\left.+L_{0}\left(e^{\left(i \omega_{1}+i \omega_{2}\right)} \cdot I_{d}\right)\right]^{-1} F_{1010}, & \\
-\frac{1}{\sqrt{2 l \pi}} e^{\left(i \omega_{1}+i \omega_{2}\right) \theta}\left[-\left(i \omega_{1}+i \omega_{2}\right)-\frac{\left(n_{2}-n_{1}\right)^{2}}{l^{2}} D_{0}\right. & \text { otherwise, } \\
\left.+L_{0}\left(e^{\left(i \omega_{1}+i \omega_{2}\right)} \cdot I_{d}\right)\right]^{-1} F_{1010}, &
\end{array}\right. \\
& h_{00020}(\theta)=-\frac{1}{\sqrt{l \pi}} e^{2 i \omega_{2} \theta}\left[-2 i \omega_{2}+L_{0}\left(e^{2 i \omega_{2} \cdot} I_{d}\right)\right]^{-1} F_{0020} \\
& h_{2 n_{2} 0011}(\theta)=-\frac{1}{\sqrt{2 l \pi}}\left[-\frac{\left(2 n_{2}\right)^{2}}{l^{2}} D_{0}+L_{0}\left(I_{d}\right)\right]^{-1} F_{0011}, \\
& h_{2 n_{2} 0020}(\theta)=-\frac{1}{\sqrt{2 l \pi}} e^{2 i \omega_{2} \theta}\left[-2 i \omega_{2}-\frac{\left(2 n_{2}\right)^{2}}{l^{2}} D_{0}+L_{0}\left(e^{2 i \omega_{2} \cdot} I_{d}\right)\right]^{-1} F_{0020}, \\
& h_{2 n_{2} 1100}(\theta)=\left\{\begin{array}{cc}
-\frac{1}{\sqrt{2 l \pi}}\left[-\frac{\left(2 n_{2}\right)^{2}}{l^{2}} D_{0}+L_{0}\left(I_{d}\right)\right]^{-1} F_{1100}, & n_{2}=n_{1}, \\
0, & n_{2} \neq n_{1},
\end{array}\right. \\
& h_{n_{1}+n_{2} 0110}(\theta)=-\frac{1}{\sqrt{2 l \pi}} e^{\left(-i \omega_{1}+i \omega_{2}\right) \theta}\left[-\left(-i \omega_{1}+i \omega_{2}\right)\right. \\
& \left.-\frac{\left(n_{1}+n_{2}\right)^{2}}{l^{2}} D_{0}+L_{0}\left(e^{\left(-i \omega_{1}+i \omega_{2}\right)} \cdot I_{d}\right)\right]^{-1} F_{0110},
\end{aligned}
$$




$$
h_{n_{2}-n_{1} 0110}(\theta)=\left\{\begin{array}{c}
-\frac{1}{\sqrt{l \pi}} e^{\left(-i \omega_{1}+i \omega_{2}\right) \theta}\left[-\left(-i \omega_{1}+i \omega_{2}\right)\right. \\
\left.\quad+L_{0}\left(e^{\left(-i \omega_{1}+i \omega_{2}\right) \cdot} I_{d}\right)\right]^{-1} F_{0110}, n_{2}=n_{1}, \\
\frac{1}{2 i \omega_{1}-i \omega_{2}} \phi_{1}(\theta) f_{0110}^{1(1)}+\frac{1}{-i \omega_{2}} \phi_{2}(\theta) f_{0110}^{1(2)} \\
-\frac{1}{\sqrt{2 l \pi}} e^{\left(-i \omega_{1}+i \omega_{2}\right) \theta}\left[-\left(-i \omega_{1}+i \omega_{2}\right)-\frac{\left(n_{2}-n_{1}\right)^{2}}{l^{2}} D_{0}\right. \\
\left.\quad+L_{0}\left(e^{\left(-i \omega_{1}+i \omega_{2}\right) \cdot} I_{d}\right)\right]^{-1} F_{0110}, n_{2}=2 n_{1}, \\
-\frac{1}{\sqrt{2 l \pi}} e^{\left(-i \omega_{1}+i \omega_{2}\right) \theta}\left[-\left(-i \omega_{1}+i \omega_{2}\right)-\frac{\left(n_{2}-n_{1}\right)^{2}}{l^{2}} D_{0}\right. \\
\left.\quad+L_{0}\left(e^{\left(-i \omega_{1}+i \omega_{2}\right)} I_{d}\right)\right]^{-1} F_{0110}, \text { otherwise. }
\end{array}\right.
$$

Hence, by (27), (40), (46), and (49), we have

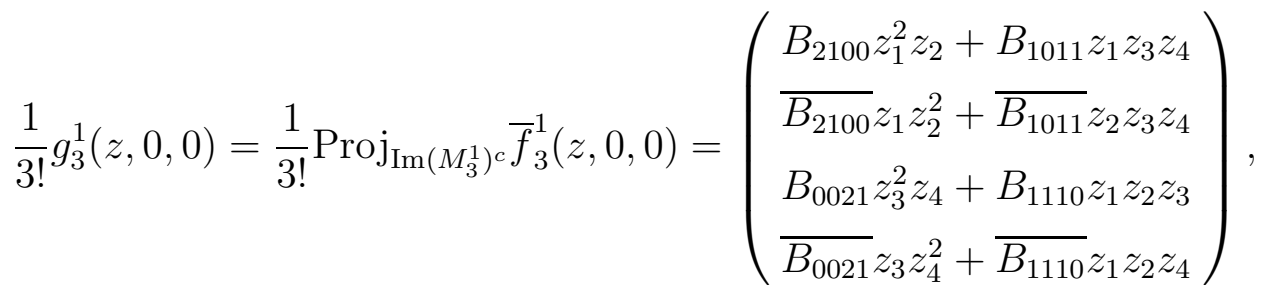

with

$$
\begin{array}{ll}
B_{2100}=C_{2100}+\frac{3}{2}\left(D_{2100}+E_{2100}\right), & B_{1011}=C_{1011}+\frac{3}{2}\left(D_{1011}+E_{1011}\right), \\
B_{0021}=C_{0021}+\frac{3}{2}\left(D_{0021}+E_{0021}\right), & B_{1110}=C_{1110}+\frac{3}{2}\left(D_{1110}+E_{1110}\right) .
\end{array}
$$

Therefore, by (25) and (51), the normal form truncated to the third order for double Hopf bifurcation reads as

$$
\begin{aligned}
& \dot{z}_{1}=\quad i \omega_{1} z_{1}+B_{11} \alpha_{1} z_{1}+B_{21} \alpha_{2} z_{1}+B_{2100} z_{1}^{2} z_{2}+B_{1011} z_{1} z_{3} z_{4}, \\
& \dot{z}_{2}=-i \omega_{1} z_{2}+\overline{B_{11}} \alpha_{1} z_{2}+\overline{B_{21}} \alpha_{2} z_{2}+\overline{B_{2100}} z_{1} z_{2}^{2}+\overline{B_{1011}} z_{2} z_{3} z_{4}, \\
& \dot{z}_{3}=i \omega_{2} z_{3}+B_{13} \alpha_{1} z_{3}+B_{23} \alpha_{2} z_{3}+B_{0021} z_{3}^{2} z_{4}+B_{1110} z_{1} z_{2} z_{3}, \\
& \dot{z}_{4}=-i \omega_{2} z_{4}+\overline{B_{13}} \alpha_{1} z_{4}+\overline{B_{23}} \alpha_{2} z_{4}+\overline{B_{0021}} z_{3} z_{4}^{2}+\overline{B_{1110}} z_{1} z_{2} z_{4} .
\end{aligned}
$$

Remark 3. To sum up, the whole calculation process above can be accomplished by following three steps.

(1) Obtain the double Hopf bifurcation point by analyzing the associate characteristic equation, and find $n_{1}, n_{2}$. Rewrite the original system into the form as (8), and calculate $D_{0}, L_{0}, D_{1}^{(1,0)}, D_{1}^{(0,1)}, L_{1}^{(1,0)}$, and $L_{1}^{(0,1)}$. Calculate the eigenfunctions $\phi_{i}$ and $\psi_{i}(i=1,2,3,4)$.

(2) Calculate $B_{11}, B_{21}, B_{13}$, and $B_{23}$ from (26).

(3) Calculate $F_{m n i j}(m+n+i+j=3)$, and we can get $C_{2100}, C_{1011}, C_{0021}$, and $C_{1110}$ from (28); Calculate $F_{m n i j}(m+n+i+j=2)$, and we obtain $D_{2100}, D_{1011}, D_{0021}$, and $D_{1110}$ from (41)-(44); Calculate $f_{m n i j}^{1(k)}(m+n+i+j=2, k=1,2,3,4)$ by (30)-(39), establish the 
TABLE I. The twelve unfoldings of system (54).

\begin{tabular}{|c|c|c|c|c|c|c|c|c|c|c|c|c|}
\hline Case & Ia & $\mathrm{Ib}$ & II & III & IVa & $\mathrm{IVb}$ & V & $\mathrm{VIa}$ & VIb & VIIa & VIIb & VIII \\
\hline$d_{0}$ & +1 & +1 & +1 & +1 & +1 & +1 & -1 & -1 & -1 & -1 & -1 & -1 \\
\hline$b_{0}$ & + & + & + & - & - & - & + & + & + & - & - & - \\
\hline$c_{0}$ & + & + & - & + & - & - & + & - & - & + & + & - \\
\hline$d_{0}-b_{0} c_{0}$ & + & - & + & + & + & - & - & + & - & + & - & - \\
\hline
\end{tabular}

linear operators $S_{y z_{i}}(i=1,2,3,4)$, we can get $E_{2100}, E_{1011}, E_{0021}$, and $E_{1110}$ by the formulas in step3 in three different cases. Finally, by (52), we can get $B_{2100}, B_{1011}, B_{0021}$, and $B_{1110}$.

Make double polar coordinates transformation by

$$
\begin{aligned}
& z_{1}=r_{1} \cos \theta_{1}+i r_{1} \sin \theta_{1}, \\
& z_{2}=r_{1} \cos \theta_{1}-i r_{1} \sin \theta_{1}, \\
& z_{3}=r_{2} \cos \theta_{2}+i r_{2} \sin \theta_{2}, \\
& z_{4}=r_{2} \cos \theta_{2}-i r_{2} \sin \theta_{2},
\end{aligned}
$$

where $r_{1}, r_{2}>0$. Define $\epsilon_{1}=\operatorname{Sign}\left(\operatorname{Re} B_{2100}\right), \epsilon_{2}=\operatorname{Sign}\left(\operatorname{Re} B_{0021}\right)$, carry out the rescaling $\widehat{r}_{1}=r_{1} \sqrt{\left|B_{2100}\right|}, \widehat{r}_{2}=r_{2} \sqrt{\left|B_{0021}\right|}, \widehat{t}=t \epsilon_{1}$, and drop the hats, then system (153) becomes

$$
\begin{aligned}
& \dot{r}_{1}=r_{1}\left(c_{1}+r_{1}^{2}+b_{0} r_{2}^{2}\right), \\
& \dot{r}_{2}=r_{2}\left(c_{2}+c_{0} r_{1}^{2}+d_{0} r_{2}^{2}\right),
\end{aligned}
$$

where

$$
\begin{aligned}
& c_{1}=\epsilon_{1}\left(\operatorname{Re} B_{11} \alpha_{1}+\operatorname{Re} B_{21} \alpha_{2}\right)=\epsilon_{1}\left(\operatorname{Re} B_{11}\left(\mu_{1}-\mu_{0,1}\right)+\operatorname{Re} B_{21}\left(\mu_{2}-\mu_{0,2}\right)\right), \\
& \left.c_{2}=\epsilon_{1}\left(\operatorname{Re} B_{13} \alpha_{1}+\operatorname{Re} B_{23} \alpha_{2}\right)=\epsilon_{1}\left(\operatorname{Re} B_{13}\left(\mu_{1}-\mu_{0,1}\right)+\operatorname{Re} B_{23}\left(\mu_{2}-\mu_{0,2}\right)\right)\right), \\
& b_{0}=\frac{\epsilon_{1} \epsilon_{2} \operatorname{Re} B_{1011}}{\operatorname{Re} B_{0021}}, c_{0}=\frac{\operatorname{Re} B_{1110}}{\operatorname{Re} B_{2100}}, d_{0}=\epsilon_{1} \epsilon_{2} .
\end{aligned}
$$

By chapter 7.5 in [25], Eq. (54) has twelve distinct kinds of unfoldings (see Table 1).

Remark 4. In section 4, case Ib appears, thus we draw bifurcation set and phase portraits for the unfolding of case Ib in Figure 1 [25]. For case Ib, near the double Hopf bifurcation point, the $\left(\alpha_{1}, \alpha_{2}\right)$ plane is divided into six regions D1-D6. In region D1, the equilibrium is a sink; when the parameters vary and enter the region D2 (or D6), the stable equilibrium bifurcates into a stable periodic solution via supercritical Hopf bifurcations. For parameters 


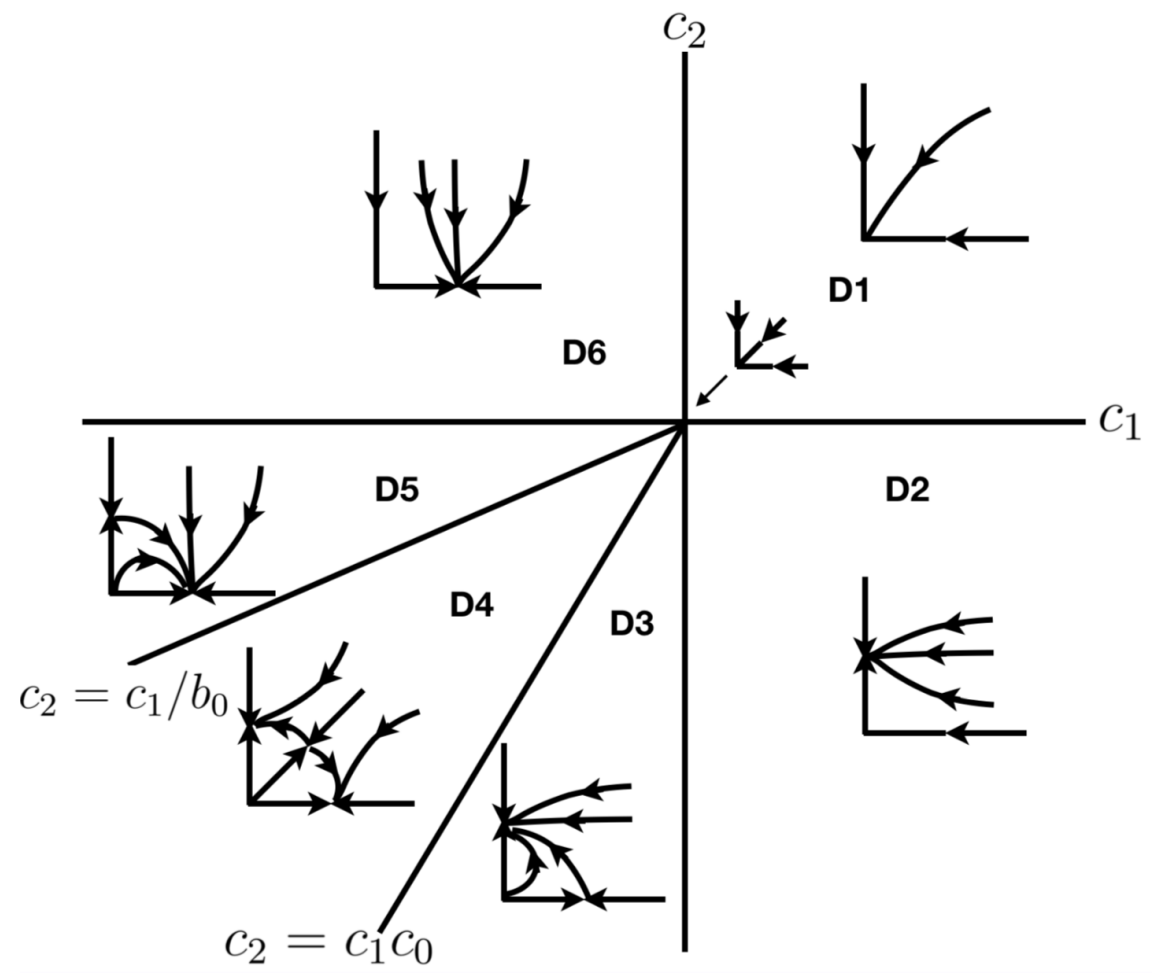

FIG. 1. Phase portraits for the unfoldings of case Ib with $\epsilon_{1}=-1$.

in D3 (or D5), periodic solutions appear via secondary supercritical Hopf bifurcations, but they are saddle type and only stable on the center manifold; when the parameters cross the Hopf bifurcation curve $c_{2}=c_{1} c_{0}$ (or $c_{2}=c_{1} / b_{0}$ ) into the region $D 4$, there are two stable periodic solutions coexisting in $\mathrm{D} 4$.

Remark 5. In section 5 case VIa arises, thus we draw bifurcation set and phase portraits for the unfolding of case VIa in Figure 20 [25]. For case VIa, near the double Hopf bifurcation point, the $\left(\alpha_{1}, \alpha_{2}\right)$ plane is divided into eight regions D1-D8. In region D2, the positive equilibrium is a sink; In region D3, there is a stable periodic solution. In D4, there is a quasi-periodic solution on the two-dimensional torus; In region D5, there is a quasi-periodic solution on the three-dimensional torus. When the parameters vary and enter D6, threedimensional torus vanish. Generally, a vanishing 3-torus might accompany the phenomenon of chaos [4, 16, 47], so near the double Hopf bifurcation point, strange attractors may exist. 


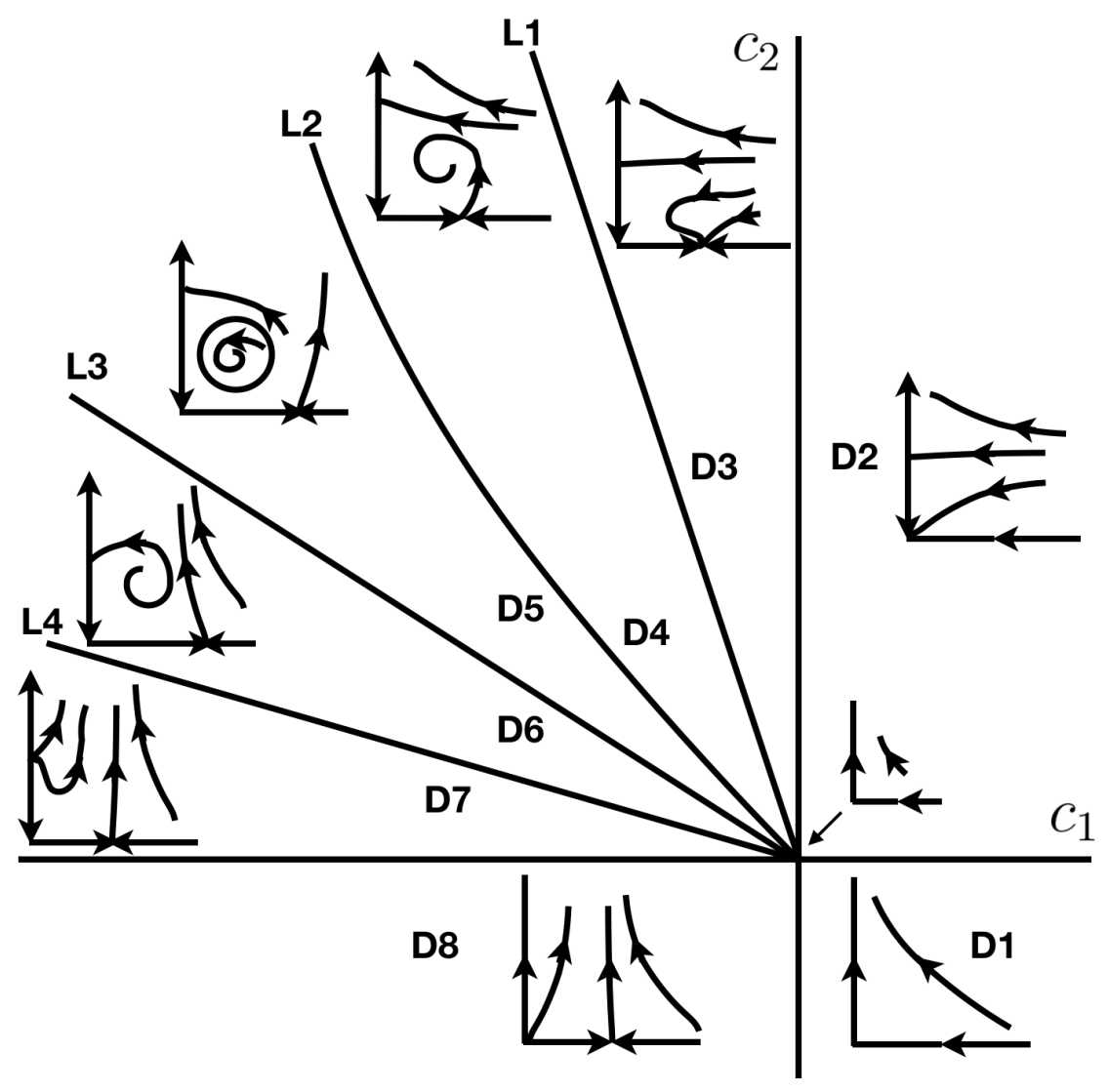

FIG. 2. Phase portraits for the unfoldings of case VIa with $\epsilon_{1}=-1$, where $L_{1}: c_{2}=c_{0} c_{1}$, $L_{2}: c_{2}=\frac{c_{0}-1}{b_{0}+1} c_{1}+o\left(c_{1}\right), L_{3}: c_{2}=\frac{c_{0}-1}{b_{0}+1} c_{1}, L_{4}: c_{2}=-\frac{1}{b_{0}} c_{1}$.

\section{APPLICATION TO A DIFFUSIVE EPIDEMIC MODEL WITH DELAY AND STAGE STRUCTURE}

In this section, a diffusive epidemic model with delay and stage structure is considered. Taking the time delay $\omega$ and a diffusive coefficient as bifurcation parameters, we show that double Hopf bifurcation can undergo with two wave numbers in different cases, such as $n_{1}=0, n_{2} \neq 0$, and $n_{1} \neq 0, n_{2} \neq 0$. Following the steps of Remark 3 , the normal form can be calculated, and the unfolding system can be got. Simulations illustrate that the spatiotemporal dynamics turn out to be very complicated near the double Hopf bifurcation point. In some regions, there are two stable nonhomogeneous periodic solutions or a homogeneous and a nonhomogeneous periodic solution coexisting. 


\subsection{Model and the Existence of Double Hopf Bifurcation}

The stage-structured epidemic model with the maturation delay and freely-moving delay is given by

$$
\left\{\begin{aligned}
\frac{\partial S(x, t)}{\partial t}= & d_{1} \Delta S(x, t)+\alpha y(x, t)-d S(x, t)-\alpha e^{-d \tau} y(x, t-\tau) \\
& -\mu S(x, t-\omega) I(x, t)+\gamma I(x, t), \\
\frac{\partial I(x, t)}{\partial t}= & d_{2} \Delta I(x, t)+\mu S(x, t-\omega) I(x, t)-d I(x, t)-\gamma I(x, t), \\
\frac{\partial y(x, t)}{\partial t}= & d_{3} \Delta y(x, t)+\alpha e^{-d \tau} y(x, t-\tau)-\beta y^{2}(x, t), \\
\frac{\partial S(x, t)}{\partial x}= & 0, \quad \frac{\partial I(x, t)}{\partial x}=0, \quad \frac{\partial y(x, t)}{\partial x}=0, \text { at } x=0 \text { and } l \pi,
\end{aligned}\right.
$$

where $S(x, t)$ and $I(x, t)$ represent the population of susceptible and infected immature individuals, and $y(x, t)$ represents the population of mature individuals. $\tau$ is the time taken for the immature individuals to maturity, $\omega$ is the time taken for the immature individuals from birth to moving freely. $\alpha$ is the natural birth rate, $d$ is the natural death rate of the immature stage, $\beta$ is the death rate of the mature individuals of logistic nature, $\mu$ is the disease transmission rate, and $\gamma$ is the recovery rate.

Denote the basic reproduction ratio by $R_{0}=\frac{\mu \alpha^{2} e^{-d \tau}\left(1-e^{-d \tau}\right)}{d \beta(d+\gamma)}$. We can easily verify that if $R_{0}>1$, system (55) has a positive constant equilibrium $E^{*}\left(S^{*}, I^{*}, y^{*}\right)=\left(\frac{d+\gamma}{\mu}, \frac{(d+\gamma)}{\mu}\left(R_{0}-\right.\right.$ 1), $\left.\frac{\alpha e^{-d \tau}}{\beta}\right)[15]$.

Linearizing system (55) at the positive equilibrium $E^{*}\left(S^{*}, I^{*}, y^{*}\right)$, we have

$$
\frac{\partial}{\partial t}\left(\begin{array}{l}
S(x, t) \\
I(x, t) \\
y(x, t)
\end{array}\right)=(D \Delta+A)\left(\begin{array}{l}
S(x, t) \\
I(x, t) \\
y(x, t)
\end{array}\right)+G_{1}\left(\begin{array}{c}
S(x, t-\omega) \\
I(x, t-\omega) \\
y(x, t-\omega)
\end{array}\right)+G_{2}\left(\begin{array}{c}
S(x, t-\tau) \\
I(x, t-\tau) \\
y(x, t-\tau)
\end{array}\right)
$$

where $D=\operatorname{diag}\left\{d_{1}, d_{2}, d_{3}\right\}$,

$$
A=\left(\begin{array}{ccc}
-d & -\mu S^{*}+\gamma & \alpha \\
0 & \mu S^{*}-d-\gamma & 0 \\
0 & 0 & -2 \beta y^{*}
\end{array}\right), G_{1}=\left(\begin{array}{ccc}
-\mu I^{*} & 0 & 0 \\
\mu I^{*} & 0 & 0 \\
0 & 0 & 0
\end{array}\right), G_{2}=\left(\begin{array}{ccc}
0 & 0 & -\alpha e^{-d \tau} \\
0 & 0 & 0 \\
0 & 0 & \alpha e^{-d \tau}
\end{array}\right)
$$

The corresponding characteristic equation is

$$
\operatorname{det}\left(\lambda I_{3}-M_{n}-A-G_{1} e^{-\lambda \omega}-G_{2} e^{-\lambda \tau}\right)=0,
$$


where $I_{3}$ is the $3 \times 3$ identity matrix and $M_{n}=-\frac{n^{2}}{l^{2}} D, n \in \mathbb{N}_{0}$. That is, each characteristic value $\lambda$ is a root of an equation

$$
\left(\lambda-\alpha e^{-d \tau} e^{-\lambda \tau}+2 \beta y^{*}+d_{3} \frac{n^{2}}{l^{2}}\right) \cdot \Delta_{n}(\lambda, \tau)=0,
$$

where

$$
\Delta_{n}(\lambda, \tau)=\lambda^{2}+\left(d+d_{1} \frac{n^{2}}{l^{2}}+d_{2} \frac{n^{2}}{l^{2}}\right) \lambda+d_{2} \frac{n^{2}}{l^{2}}\left(d+d_{1} \frac{n^{2}}{l^{2}}\right)+e^{-\lambda \omega}\left(\mu I^{*} \lambda+\mu I^{*} d_{2} \frac{n^{2}}{l^{2}}+\mu I^{*} d\right),
$$

with $n \in \mathbb{N}_{0}$.

We can easily prove that the roots of $\lambda-\alpha e^{-d \tau} e^{-\lambda \tau}+2 \beta y^{*}+d_{3} \frac{n^{2}}{l^{2}}=0$ have negative real part. To investigate the location of the roots, it remains to consider the roots of $\Delta_{n}(\lambda, \tau)=0$.

When $\omega=0$, Eq. (57) becomes

$$
\lambda^{2}+T_{n} \lambda+J_{n}=0, \quad n \in \mathbb{N}_{0}
$$

Since $T_{n}=d+d_{1} \frac{n^{2}}{l^{2}}+d_{2} \frac{n^{2}}{l^{2}}+\mu I^{*}>0, J_{n}=d_{2} \frac{n^{2}}{l^{2}}\left(d+d_{1} \frac{n^{2}}{l^{2}}\right)+\mu I^{*} d_{2} \frac{n^{2}}{l^{2}}+\mu I^{*} d>0$, we know that all roots of Eq. (58) have negative real part, and thus so do the roots of Eq. (56) for $\omega=0$ when $R_{0}>1$.

By substituting $i z(z>0)$ into Eq. (57), and separating the real and imaginary parts, we have

$$
\left\{\begin{array}{l}
-z^{2}+d_{2} \frac{n^{2}}{l^{2}}\left(d+d_{1} \frac{n^{2}}{l^{2}}\right)=-\mu I^{*} z \sin \omega z-\left(\mu I^{*} d_{2} \frac{n^{2}}{l^{2}}+\mu I^{*} d\right) \cos \omega z, \\
\left(d+d_{1} \frac{n^{2}}{l^{2}}+d_{2} \frac{n^{2}}{l^{2}}\right) z=-\mu I^{*} z \cos \omega z+\left(\mu I^{*} d_{2} \frac{n^{2}}{l^{2}}+\mu I^{*} d\right) \sin \omega z
\end{array}\right.
$$

which is solved by

$$
\begin{aligned}
& \sin z_{n} \omega=\frac{\left(d+d_{1} \frac{n^{2}}{l^{2}}+d_{2} \frac{n^{2}}{l^{2}}\right)\left(\mu I^{*} d_{2} \frac{n^{2}}{l^{2}}+\mu I^{*} d\right) z_{n}-\left[d_{2} \frac{n^{2}}{l^{2}}\left(d+d_{1} \frac{n^{2}}{l^{2}}\right)-z_{n}^{2}\right] \mu I^{*} z_{n}}{\left(\mu I^{*} z_{n}\right)^{2}+\left(\mu I^{*} d_{2} \frac{n^{2}}{l^{2}}+\mu I^{*} d\right)^{2}} \\
& \triangleq S_{n}\left(z_{n}\right), \\
& \cos z_{n} \omega=-\frac{\left(d+d_{1} \frac{n^{2}}{l^{2}}+d_{2} \frac{n^{2}}{l^{2}}\right) \mu I^{*} z_{n}^{2}+\left[d_{2} \frac{n^{2}}{l^{2}}\left(d+d_{1} \frac{n^{2}}{l^{2}}\right)-z_{n}^{2}\right]\left(\mu I^{*} d_{2} \frac{n^{2}}{l^{2}}+\mu I^{*} d\right)}{\left(\mu I^{*} d_{2} \frac{n^{2}}{l^{2}}+\mu I^{*} d\right)^{2}+\left(\mu I^{*} z_{n}\right)^{2}} \\
& \triangleq C_{n}\left(z_{n}\right) .
\end{aligned}
$$

Then, we obtain

$$
G(z)=z^{4}+P_{n} z^{2}+Q_{n}=0,
$$

where

$$
\begin{aligned}
& P_{n}=\left(d_{2} \frac{n^{2}}{l^{2}}\right)^{2}+\left(d+d_{1} \frac{n^{2}}{l^{2}}+\mu I^{*}\right)\left(d+d_{1} \frac{n^{2}}{l^{2}}-\mu I^{*}\right), \\
& Q_{n}=J_{n} K_{n}=J_{n}\left(d_{2} \frac{n^{2}}{l^{2}}\left(d+d_{1} \frac{n^{2}}{l^{2}}\right)-\mu I^{*} d_{2} \frac{n^{2}}{l^{2}}-\mu I^{*} d\right) .
\end{aligned}
$$


Noticing that $J_{n}>0$, the sign of $Q_{n}$ coincides with that of $K_{n}$. Since $K_{0}=-\mu I^{*} d<0$, and $K_{n}$ is a quadratic polynomial with respect to $n^{2}$, we can conclude that there exists $k_{1} \in \mathbb{N}_{0}$, such that

$$
\begin{array}{ll}
K_{n}<0 & \text { for } 0 \leq n \leq k_{1}, \\
K_{n}>0 & \text { for } n \geq k_{1}+1, n \in \mathbb{N}_{0} .
\end{array}
$$

Denote the positive real root of the equation $K_{n}=0$ by $n_{2}\left(k_{1}<n_{2}<k_{1}+1\right)$, then we have $K_{n_{2}}=d_{1} d_{2} \frac{1}{l^{4}} n_{2}^{4}+\left(d-\mu I^{*}\right) d_{2} \frac{1}{l^{2}} n_{2}^{2}-\mu I^{*} d=0$. Since $-\mu I^{*} d<0$, we have $d_{1} d_{2} \frac{1}{l^{4}} n_{2}^{4}+$ $\left(d-\mu I^{*}\right) d_{2} \frac{1}{l^{2}} n_{2}^{2}=\left(d+d_{1} \frac{n_{2}^{2}}{l^{2}}-\mu I^{*}\right) d_{2} \frac{1}{l^{2}} n_{2}^{2}>0$. It means that

$$
d+d_{1} \frac{n_{2}^{2}}{l^{2}}-\mu I^{*}>0
$$

By (61), we have

$$
P_{n_{2}}=\left(d_{2} \frac{n_{2}^{2}}{l^{2}}\right)^{2}+\left(d+d_{1} \frac{n_{2}^{2}}{l^{2}}+\mu I^{*}\right)\left(d+d_{1} \frac{n_{2}^{2}}{l^{2}}-\mu I^{*}\right)>0
$$

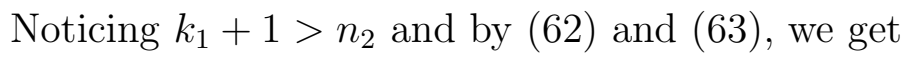

$$
P_{n}>0, \text { for } n \geq k_{1}+1, k_{1} \in \mathbb{N}_{0} .
$$

From (62) and (64), we can conclude that for $n \in \mathbb{N}_{0}$ and $n \leq k_{1}$, (60) has only one positive real root

$$
z_{n}=\sqrt{\frac{-P_{n}+\sqrt{P_{n}^{2}-4 Q_{n}}}{2}}
$$

For $n \in \mathbb{N}_{0}$ and $n \geq k_{1}+1$, (60) has no positive real roots.

In fact,

$$
S_{n}\left(z_{n}\right)=\frac{z_{n}\left\{\left(d+d_{1} \frac{n^{2}}{l^{2}}+d_{2} \frac{n^{2}}{l^{2}}\right)\left(\mu I^{*} d_{2} \frac{n^{2}}{l^{2}}+\mu I^{*} d\right)-\left[d_{2} \frac{n^{2}}{l^{2}}\left(d+d_{1} \frac{n^{2}}{l^{2}}\right)\right] \mu I^{*}+z_{n}^{2} \mu I^{*}\right\}}{\left(\mu I^{*} z_{n}\right)^{2}+\left(\mu I^{*} d_{2} \frac{n^{2}}{l^{2}}+\mu I^{*} d\right)^{2}},
$$

where $\left(d+d_{1} \frac{n^{2}}{l^{2}}+d_{2} \frac{n^{2}}{l^{2}}\right)\left(\mu I^{*} d_{2} \frac{n^{2}}{l^{2}}+\mu I^{*} d\right)-\left[d_{2} \frac{n^{2}}{l^{2}}\left(d+d_{1} \frac{n^{2}}{l^{2}}\right)\right] \mu I^{*}=\mu I^{*}\left[d\left(d+d_{1} \frac{n^{2}}{l^{2}}+d_{2} \frac{n^{2}}{l^{2}}\right)+\right.$ $\left.\left(d_{2} \frac{n^{2}}{l^{2}}\right)^{2}\right]>0$. Thus, when $n \in\left\{0,1, \cdots, k_{1}\right\}, S_{n} \geq 0$, define

$$
\omega_{n}^{j}=\frac{\arccos C_{n}\left(z_{n}\right)+2 j \pi}{z_{n}} .
$$

Differentiating the two sides of Eq. (57) with respective to $\omega$, Using (57) and (59), we obtain

$$
\left.\frac{\mathrm{dRe} \lambda(\omega)}{\mathrm{d} \omega}\right|_{\omega=\omega_{n}^{j}}=\frac{\sqrt{P_{n}^{2}-4 Q_{n}}}{\left(\mu I^{*}\right)^{2} z_{n}^{2}+\left(\mu I^{*} d_{2} \frac{n^{2}}{l^{2}}+\mu I^{*} d\right)^{2}}>0 .
$$


From (66), we have the very first critical value as

$$
\omega^{*}=\omega_{n_{0}}^{0}=\min _{n \in\left\{0,1, \cdots, k_{1}\right\}}\left\{\omega_{n}^{0}\right\}, \quad z^{*}=z_{n_{0}} .
$$

Due to the general Hopf bifurcation theorem [18, 53], we have the following theorem.

Theorem 1. Suppose $R_{0}>1$.

(1) The equilibrium $E^{*}$ of system (55) is locally asymptotically stable for $0 \leq \omega<\omega_{n_{0}}^{0}$ and is unstable for $\omega>\omega_{n_{0}}^{0}$.

(2) System (55) undergoes a Hopf bifurcation at the equilibrium $E^{*}$ when $\omega=\omega_{n}^{j}$, for $j \in \mathbb{N}_{0}$ and $n \in\left\{0,1, \cdots, k_{1}\right\}$.

Now we need to give a condition under which a double Hopf bifurcation occurs. From (66) and (67), we have that when $n \in\left\{0,1, \cdots, k_{1}\right\}, \omega_{n}^{0}=\frac{\arccos C_{n}\left(z_{n}\right)}{z_{n}}$, and $\left.\frac{\mathrm{dRe} \lambda(\omega)}{\mathrm{d} \omega}\right|_{\omega=\omega_{n}^{0}}>0$, which means that if we vary the coefficients $d_{2}$ and $\omega$, and fix other coefficients in Eq. (55), there are $k_{1}+1$ Hopf bifurcation curves on the $d_{2}-\omega$ plane. On every Hopf bifurcation curve $\omega_{n}^{0}$, the characteristic equation (56) always has one pair of eigenvalues $\pm i z_{n}$, which crosses transversely the imaginary axis when the parameters cross the Hopf bifurcation curve, and the rest eigenvalues have non-zero real part. If we can find the intersection of two certain Hopf bifurcation curves $\omega_{n_{1}}^{0}$ and $\omega_{n_{2}}^{0}$, there must exist two pairs of eigenvalues $\pm i z_{n_{1}}$ and $\pm i z_{n_{2}}$ at the intersection point, and all the other eigenvalues have non-zero real part. Thus, a double Hopf singularity can be found by searching for the intersection of the Hopf bifurcation curves, which can be done by the following process. Firstly, for $n_{1}, n_{2} \in\left\{0,1, \cdots, k_{1}\right\}$, we regard $z_{n_{1}}$ and $z_{n_{2}}$ as functions of $d_{2}$ from Eq. (65). Secondly, the expression of $\omega_{n_{1}}^{0}$ and $\omega_{n_{2}}^{0}$ are obtained from (66). Finally, from $\omega_{n_{1}}^{0}=\omega_{n_{2}}^{0}$, we can solve the value of $d_{2}$, denoted by $d_{2}^{*}$, such that $\omega_{n_{1}}^{0}=\omega_{n_{2}}^{0}$. Thus, we have that when $d_{2}=d_{2}^{*}, \omega=\omega_{n_{1}}^{0}=\omega_{n_{2}}^{0}$, the Hopf bifurcation curves $\omega_{n_{1}}^{0}$ and $\omega_{n_{2}}^{0}$ intersect. Thus, system (55) undergoes double Hopf bifurcation at the intersection.

Theorem 2. Suppose that $R_{0}>1$ and there exists $d_{2}^{*}, n_{1}, n_{2}$, such that when $d_{2}=d_{2}^{*}$, $\omega_{n_{1}}^{0}=\omega_{n_{2}}^{0}$. Then system (55) undergoes a double Hopf bifurcation at $E^{*}$ when $d_{2}=d_{2}^{*}$, $\omega=\omega_{n_{1}}^{0}=\omega_{n_{2}}^{0} \triangleq \omega^{*}$. 


\subsection{Normal Form of Double Hopf Bifurcation}

Let $u_{1}(x, t)=S(x, \omega t)-S^{*}, u_{2}(x, t)=I(x, \omega t)-I^{*}, u_{3}(x, t)=y(x, \omega t)-y^{*}$. Thus, these transformations not only transform the equilibrium $\left(S^{*}, I^{*}, y^{*}\right)$ into $(0,0,0)$, but also normalize the delay $\omega$ to 1 , and transform the other delay $\tau$ into $\tau / \omega$. Denote $U(t)=$ $\left(u_{1}(x, t), u_{2}(x, t), u_{3}(x, t)\right)^{T}$, and $\omega=\omega^{*}+\alpha_{1}, d_{2}=d_{2}^{*}+\alpha_{2}$, then system (55) can be transformed into

$$
\begin{aligned}
\frac{\mathrm{d} U(t)}{\mathrm{d} t}=D\left(\omega^{*}+\alpha_{1}, d_{2}^{*}+\alpha_{2}\right) \Delta U(t) & +L\left(\omega^{*}+\alpha_{1}, d_{2}^{*}+\alpha_{2}\right)\left(U^{t}\right) \\
& +F\left(\omega^{*}+\alpha_{1}, d_{2}^{*}+\alpha_{2}, U^{t}\right),
\end{aligned}
$$

where

$$
\begin{aligned}
& D\left(\omega^{*}+\alpha_{1}, d_{2}^{*}+\alpha_{2}\right)=\left(\omega^{*}+\alpha_{1}\right)\left(\begin{array}{ccc}
d_{1} & 0 & 0 \\
0 & d_{2}^{*}+\alpha_{2} & 0 \\
0 & 0 & d_{3}
\end{array}\right)=D_{0}+\alpha_{1} D_{1}^{(1,0)}+\alpha_{2} D_{1}^{(0,1)}, \\
& L\left(\omega^{*}+\alpha_{1}, d_{2}^{*}+\alpha_{2}\right) U^{t}=\left(L_{0}+\alpha_{1} L_{1}^{(1,0)}+\alpha_{2} L_{1}^{(0,1)}\right) U^{t} \\
& F\left(\omega^{*}+\alpha_{1}, d_{2}^{*}+\alpha_{2}, U^{t}\right)=\left(\omega^{*}+\alpha_{1}\right)\left(\begin{array}{c}
-\mu U_{1}^{t}(-1) U_{2}^{t}(0) \\
\mu U_{1}^{t}(-1) U_{2}^{t}(0) \\
-\beta U_{3}^{t 2}(0)
\end{array}\right)
\end{aligned}
$$

with

$$
\begin{gathered}
D_{0}=\omega^{*}\left(\begin{array}{ccc}
d_{1} & 0 & 0 \\
0 & d_{2}^{*} & 0 \\
0 & 0 & d_{3}
\end{array}\right), D_{1}^{(1,0)}=\left(\begin{array}{ccc}
d_{1} & 0 & 0 \\
0 & d_{2}^{*} & 0 \\
0 & 0 & d_{3}
\end{array}\right), D_{1}^{(0,1)}=\omega^{*}\left(\begin{array}{ccc}
0 & 0 & 0 \\
0 & 1 & 0 \\
0 & 0 & 0
\end{array}\right), \\
L_{0} U^{t}=\omega^{*}\left[A U^{t}(0)+G_{1} U^{t}(-1)+G_{2} U^{t}\left(-\tau^{*}\right)\right], \\
L_{1}^{(1,0)} U^{t}=\left[A U^{t}(0)+G_{1} U^{t}(-1)+G_{2} U^{t}\left(-\tau^{*}\right)\right], \\
L_{1}^{(0,1)} U^{t}=0,
\end{gathered}
$$

and

$$
\tau^{*}=\tau / \omega
$$

Eq. (68) can be rewritten as

$$
\frac{d U}{d t}=D_{0} \Delta U(t)+L_{0} U^{t}+\widetilde{F}\left(\alpha, U^{t}\right),
$$


where

$$
\widetilde{F}\left(\alpha, U^{t}\right)=\alpha_{1} D_{1}^{(1,0)} \Delta U+\alpha_{2} D_{1}^{(0,1)} \Delta U+\alpha_{1} L_{1}^{(1,0)} U^{t}+F\left(\omega^{*}+\alpha_{1}, d_{2}^{*}+\alpha_{2}, U^{t}\right)
$$

Consider the linearized system of (69)

$$
\frac{d U}{d t}=D_{0} \Delta U(t)+L_{0} U^{t}
$$

From the previous discussion, we know that system (71) has pure imaginary eigenvalues $\left\{ \pm i z_{n_{1}} \omega^{*}, \pm i z_{n_{2}} \omega^{*}\right\}$ at the double Hopf bifurcation point and the other eigenvalues with non-zero real part.

Assume that the non-resonant condition holds true and use the algorithm we give in Section 3. After a few calculations, we have that the bases of $P_{\Lambda}$ and $P^{*}$, respectively, are $\Phi(\theta)=\left(\phi_{1}(\theta), \bar{\phi}_{1}(\theta), \phi_{3}(\theta), \bar{\phi}_{3}(\theta)\right)$, and $\Psi(s)=\left(\psi_{1}(s), \bar{\psi}_{1}(s), \psi_{3}(s), \bar{\psi}_{3}(s)\right)^{T}$, with

$$
\begin{array}{r}
\phi_{1}(\theta)=\left(1, p_{12}, p_{13}\right)^{T} e^{i z_{n_{1}} \omega^{*} \theta}, \phi_{3}(\theta)=\left(1, p_{32}, p_{33}\right)^{T} e^{i z_{n_{2}} \omega^{*} \theta}, \\
\psi_{1}^{*}(s)=D_{1}\left(1, q_{12}^{*}, q_{13}^{*}\right) e^{-i z_{n_{1}} \omega^{*} s}, \psi_{3}^{*}(s)=D_{2}\left(1, q_{32}^{*}, q_{33}^{*}\right) e^{-i z_{n_{2}} \omega^{*} s},
\end{array}
$$

where

$$
\begin{gathered}
p_{12}=\frac{\mu I^{*} e^{-i z_{n_{1}} \omega^{*}}}{d_{2} \frac{n^{2}}{l^{2}}+i z_{n_{1}}}, p_{13}=0, p_{32}=\frac{\mu I^{*} e^{-i z_{n_{2}} \omega^{*}}}{d_{2} \frac{n^{2}}{l^{2}}+i z_{n_{2}}}, p_{33}=0, \\
q_{12}^{*}=-\frac{-\mu S^{*}+\gamma}{-d_{2} \frac{n^{2}}{l^{2}}-i z_{n_{1}}}, q_{32}^{*}=-\frac{-\mu S^{*}+\gamma}{-d_{2} \frac{n^{2}}{l^{2}}-i z_{n_{2}}}, \\
q_{13}^{*}=-\frac{\alpha-\alpha e^{-d \tau} e^{-i z_{n_{1}} \omega^{*} \tau^{*}}}{-2 \beta y^{*}-d_{3} \frac{n^{2}}{l^{2}}+\alpha e^{-d \tau} e^{-i z_{n_{1}} \omega^{*} \tau^{*}-i z_{n_{1}}}}, \\
q_{33}^{*}=-\frac{\alpha-\alpha e^{-d \tau} e^{-i z_{n_{2}} \omega^{*} \tau^{*}}}{-2 \beta y^{*}-d_{3} \frac{n^{2}}{l^{2}}+\alpha e^{-d \tau} e^{-i z_{n_{2}} \omega^{*} \tau^{*}-i z_{n_{2}}}} \\
D_{1}=\frac{1}{1+q_{12}^{*} p_{12}+\omega^{*} \mu I^{*} e^{-i z_{n} \omega^{*}}\left(q_{12}^{*}-1\right)}, D_{2}=\frac{1}{1+q_{32}^{*} p_{32}+\omega^{*} \mu I^{*} e^{-i z_{n_{2}} \omega^{*}\left(q_{32}^{*}-1\right)}} .
\end{gathered}
$$

Consider the Taylor expansion

$$
\widetilde{F}\left(\alpha, U^{t}\right)=\frac{1}{2 !} \widetilde{F}_{2}\left(\alpha, U^{t}\right)+\frac{1}{3 !} \widetilde{F}_{3}\left(\alpha, U^{t}\right)
$$

where $\widetilde{F}_{2}\left(\alpha, U^{t}\right)=2 \alpha_{1} D_{1}^{(1,0)} \Delta U+2 \alpha_{2} D_{1}^{(0,1)} \Delta U+2 \alpha_{1} L_{1}^{(1,0)} U^{t}+F_{2}\left(\omega^{*}+\alpha_{1}, d_{2}^{*}+\alpha_{2}, U^{t}\right)$, and 
$\frac{1}{3 !} \widetilde{F}_{3}\left(\alpha, U^{t}\right)=0$. By a few calculations, we have

$$
F_{2000}=2 \omega^{*}\left(\begin{array}{c}
-\mu e^{-i z_{n_{1}} \omega^{*}} p_{12} \\
\mu e^{-i z_{n_{1}} \omega^{*}} p_{12} \\
-\beta p_{13}^{2}
\end{array}\right),
$$$$
F_{1010}=2 \omega^{*}\left(\begin{array}{c}
-\mu\left(e^{-i z_{n_{1}} \omega^{*}} p_{32}+e^{-i z_{n_{2}} \omega^{*}} p_{12}\right) \\
\mu\left(e^{-i z_{n_{1}} \omega^{*}} p_{32}+e^{-i z_{n_{2}} \omega^{*}} p_{12}\right) \\
-2 \beta p_{13} p_{33}
\end{array}\right),
$$$$
F_{0200}=2 \omega^{*}\left(\begin{array}{c}
-\mu e^{i z_{n_{1}} \omega^{*}} \bar{p}_{12} \\
\mu e^{i z_{n_{1}} \omega^{*}} \bar{p}_{12} \\
-\beta \bar{p}_{13}^{2}
\end{array}\right),
$$$$
F_{0101}=2 \omega^{*}\left(\begin{array}{c}
-\mu\left(e^{i z_{n_{1}} \omega^{*}} \bar{p}_{32}+e^{i z_{n_{2}} \omega^{*}} \bar{p}_{12}\right) \\
\mu\left(e^{i z_{n_{1}} \omega^{*}} \bar{p}_{32}+e^{i z_{n_{2}} \omega^{*}} \bar{p}_{12}\right) \\
-2 \beta \bar{p}_{13} \bar{p}_{33}
\end{array}\right),
$$ 


$$
F_{0011}=2 \omega^{*}\left(\begin{array}{c}
-\mu\left(e^{-i z_{n_{2}} \omega^{*}} \bar{p}_{32}+e^{i z_{n_{2}} \omega^{*}} p_{32}\right) \\
\mu\left(e^{-i z_{n_{2}} \omega^{*}} \bar{p}_{32}+e^{i z_{n_{2}} \omega^{*}} p_{32}\right) \\
-2 \beta p_{33} \bar{p}_{33} \\
-\mu e^{i z_{n_{2}} \omega^{*}} \bar{p}_{32} \\
\mu e^{i z_{n_{2}} \omega^{*}} \bar{p}_{32} \\
-\beta \bar{p}_{33}^{2}
\end{array}\right)
$$

By Remark 2 in Section 3, define the linear operators

$$
\begin{aligned}
& S_{y z_{1}}(y)=F_{y(0) z_{1}} y(0)+F_{y(-1) z_{1}} y(-1)+F_{y\left(-\frac{\tau}{\omega^{*}}\right) z_{1}} y\left(-\frac{\tau}{\omega^{*}}\right), \\
& S_{y z_{2}}(y)=F_{y(0) z_{2}} y(0)+F_{y(-1) z_{2}} y(-1)+F_{y\left(-\frac{\tau}{\omega^{*}}\right) z_{2}} y\left(-\frac{\tau}{\omega^{*}}\right), \\
& S_{y z_{3}}(y)=F_{y(0) z_{3}} y(0)+F_{y(-1) z_{3}} y(-1)+F_{y\left(-\frac{\tau}{\omega^{*}}\right) z_{3}} y\left(-\frac{\tau}{\omega^{*}}\right), \\
& S_{y z_{4}}(y)=F_{y(0) z_{4}} y(0)+F_{y(-1) z_{4}} y(-1)+F_{y\left(-\frac{\tau}{\omega^{*}}\right) z_{4}} y\left(-\frac{\tau}{\omega^{*}}\right),
\end{aligned}
$$

where

$$
\begin{aligned}
& \begin{array}{l}
F_{y(0) z_{1}}=2 \omega^{*}\left(\begin{array}{ccc}
0 & -\mu e^{-i z_{n_{1}} \omega^{*}} & 0 \\
0 & \mu e^{-i z_{n_{1}} \omega^{*}} & 0 \\
0 & 0 & -2 \beta p_{13}
\end{array}\right), F_{y(-1) z_{1}}=2 \omega^{*}\left(\begin{array}{ccc}
-\mu p_{12} & 0 & 0 \\
\mu p_{12} & 0 & 0 \\
0 & 0 & 0
\end{array}\right), F_{y\left(-\frac{\tau}{\omega^{*}}\right) z_{1}}=0, \\
F_{y(0) z_{2}}=2 \omega^{*}\left(\begin{array}{cccc}
0 & -\mu e^{i z_{n_{1}} \omega^{*}} & 0 \\
0 & \mu e^{i z_{1} \omega^{*}} & 0 \\
0 & 0 & -2 \beta \bar{p}_{13}
\end{array}\right), F_{y(-1) z_{2}}=2 \omega^{*}\left(\begin{array}{ccc}
-\mu \bar{p}_{12} & 0 & 0 \\
\mu \bar{p}_{12} & 0 & 0 \\
0 & 0 & 0
\end{array}\right), F_{y\left(-\frac{\tau}{\omega^{*}}\right) z_{2}}=0,
\end{array}
\end{aligned}
$$

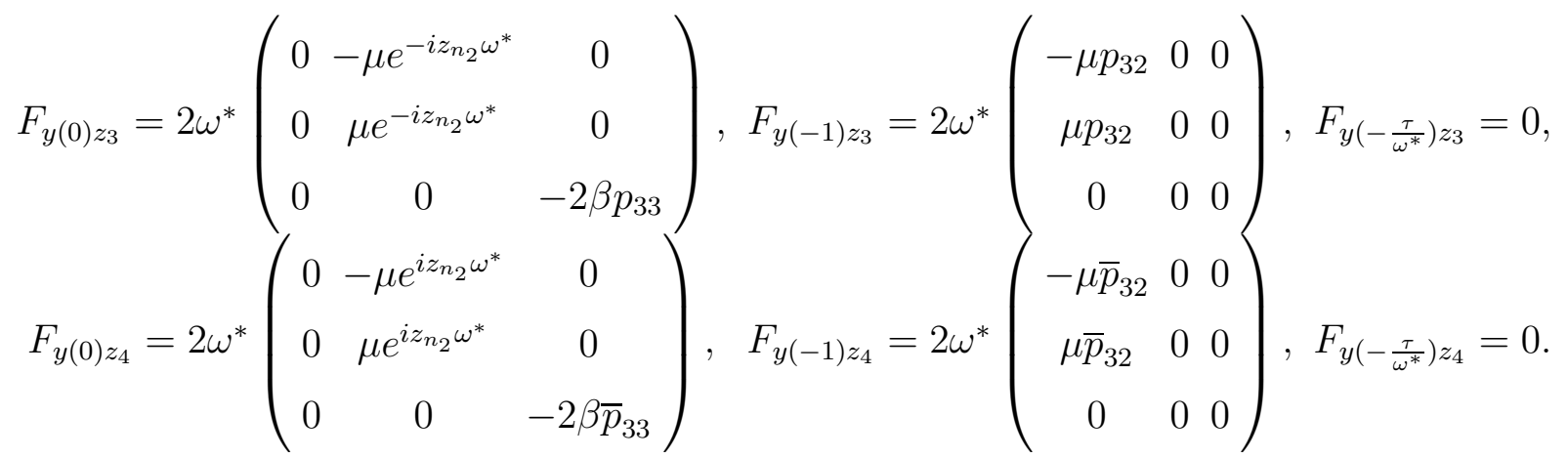

Following the steps in Remark 3, we can get all the coefficients in (53), and then obtain the normal forms up to third order. 


\subsection{Simulations}

In the following, we take $\alpha=2.1, d=0.5, \mu=0.5, \gamma=0.1, \beta=0.3, \tau=1, d_{1}=0.05$, $d_{3}=0.06$, and let $\omega$ and $d_{2}$ be the bifurcation parameters.

By (66) , we can draw the curves of Hopf bifurcation values when $d_{2}$ varies. As shown clearly in Fig. 3 a), every two Hopf bifurcation curves intersect at a double Hopf bifurcation point such as HH1, HH2 and HH3. When $d_{2}=5.23, \omega_{1}^{0}$ intersects $\omega_{2}^{0}$, and we denote the double Hopf bifurcation point HH2. It means that the wave numbers are the case of $n_{1}=1, n_{2}=2$. For HH2, we have $z_{n_{1}}=z_{1}=2.9930, z_{n_{2}}=z_{2}=3.1037$, and $\omega_{1}^{0}=$ $\omega_{2}^{0}=0.5290$. Using the algorithm we established, we get that $B_{11}=0.7184+0.5138 i, B_{21}=$ $0.0021+0.0042 i, B_{13}=0.6431+0.5805 i, B_{23}=-0.0037+0.0101 i, B_{2100}=-0.0001-0.1942 i$, $B_{1011}=-0.0010-0.3398 i, B_{0021}=-0.00071+0.00055 i$, and $B_{1110}=-0.0851-0.4349 i$. Thus, $\epsilon_{1}=-1, \epsilon_{2}=-1, b_{0}=1.4401, c_{0}=0.0016, d_{0}=1, d_{0}-b_{0} c_{0}=-0.0023$. It means that the unfolding system is of type Ib. Moreover, we have $c_{1}=-0.7184 \alpha_{1}-0.0021 \alpha_{2}, c_{2}=$ $-0.6431 \alpha_{1}+0.0037 \alpha_{2}$. Thus, the bifurcation set near HH2 is shown in Figure $3 \mathrm{~b}$ ), in which the two black lines are two pitchfork bifurcation curves $\omega=\left(d_{2}-5.23\right) /(28.2318)+0.5290$, $\omega=\left(d_{2}-5.23\right) /(-346.6577)+0.5290$. The parameter plane near the bifurcation point is divided into six regions. In D1, the positive equilibrium is asymptotically stable. In D2 or D6, there are stable periodic solutions. In region D4, there are two stable nonhomogeneous periodic solutions coexisting, which are corresponding to two different eigenfunctions. From Theorem 2.2 in Section 6.2 of [53], Hopf bifurcating periodic solutions of system (68) can be parameterized by a small parameter $\epsilon$. Thus, when $\nu=\nu(\epsilon)$ and $\epsilon$ are near 0, (i.e. when $\omega$ is near $\omega^{*}$ ), two periodic solutions have the following representations respectively

$$
\begin{aligned}
& U_{t}(\nu, \theta)(x)=\epsilon \operatorname{Re} \phi_{1}(\theta) e^{i z_{n_{1}} \omega^{*} t} \cos \frac{n_{1}}{l} x+O\left(\epsilon^{2}\right), \\
& U_{t}(\nu, \theta)(x)=\epsilon \operatorname{Re} \phi_{3}(\theta) e^{i z_{n_{2}} \omega^{*} t} \cos \frac{n_{2}}{l} x+O\left(\epsilon^{2}\right),
\end{aligned}
$$

where $\phi_{1}(\theta)$ and $\phi_{3}(\theta)$ is defined in (72). Figure 4 illustrates that the two solutions have totally different spatial shape.

For double Hopf bifurcation points $\mathrm{HH} 1$ and $\mathrm{HH} 3$, we can calculate the parameters in Table 2. Obviously, these two points are also of type Ib. We notice that during the calculation of step 3 in third order normal form of the three double Hopf bifurcation points, the cases $n_{1}=0, n_{2} \neq 0$ (e.g. HH3), and $n_{1} \neq 0, n_{2} \neq 0$ (e.g. HH1, HH2) both occur. 


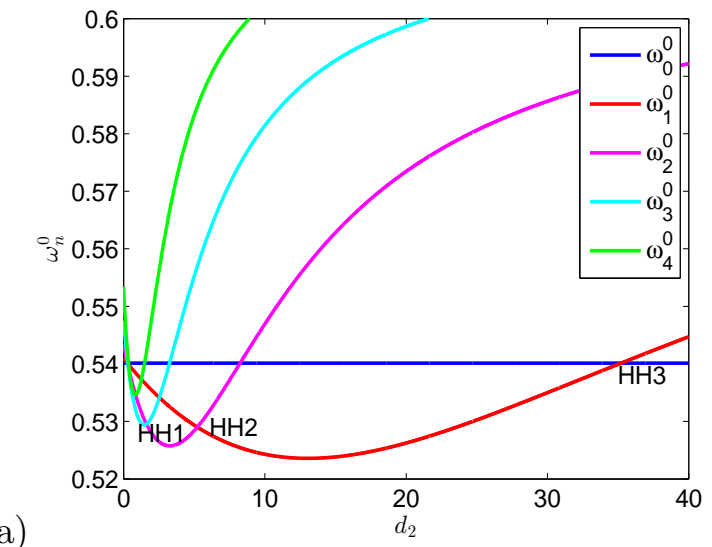

b)

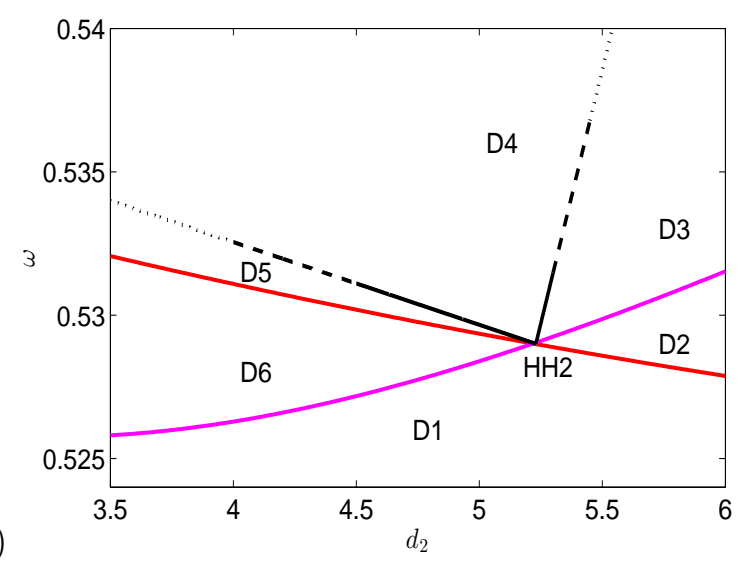

FIG. 3. a) When $\alpha=2.1, d=0.5, \mu=0.5, \gamma=0.1, \beta=0.3, \tau=1, d_{1}=0.05, d_{3}=0.06$, the bifurcation set on the $d_{2}-\omega$ plane is drawn, and double Hopf bifurcation points are marked. b) Complete bifurcation set near the double Hopf point HH2.

TABLE II. Parameter values at double Hopf bifurcation points.

\begin{tabular}{ccccccccccc}
\hline Point & $d_{2}^{*}$ & $\omega^{*}$ & $n_{1}$ & $z_{n_{1}}$ & $n_{2}$ & $z_{n_{2}}$ & $b_{0}$ & $c_{0}$ & $d_{0}$ & $d_{0}-b_{0} c_{0}$ \\
\hline HH1 & 1.62 & 0.5295 & 2 & 3.0071 & 3 & 3.0763 & 1.8774 & 1.1651 & 1 & -1.1872 \\
HH2 & 5.23 & 0.5290 & 1 & 2.9930 & 2 & 3.1037 & 1.4401 & 0.0016 & 1 & -0.0023 \\
HH3 & 35.2 & 0.5401 & 0 & 2.9082 & 1 & 3.1007 & 3.1290 & 1.4043 & 1 & -3.3939 \\
\hline
\end{tabular}

\section{APPLICATION TO A DIFFUSIVE PREDATOR-PREY MODEL WITH DELAY}

In this section, a diffusive predator-prey model with delay is considered. Taking $r_{1}$ and $\tau$ as bifurcation parameters, we can find the double Hopf bifurcation point. By the steps in Remark 3, we can obtain the normal form and get the unfolding system. We will show that the system will exhibit complex dynamical behavior near the double Hopf bifurcation point: the existence of quasi-periodic solution on a 2-torus, quasi-periodic solution on a 3-torus, and even chaos. 


\subsection{Model and the Existence of Double Hopf Bifurcation}

Let us consider the predator-prey system

$$
\left\{\begin{array}{l}
\frac{\partial X(x, t)}{\partial t}=d_{1} \Delta X(x, t)+X(x, t)\left[r_{1}-a_{11} X(x, t-\tau)-a_{12} Y(x, t)\right], \\
\frac{\partial Y(x, t)}{\partial t}=d_{2} \Delta Y(x, t)+Y(x, t)\left[-r_{2}+a_{21} X(x, t)-a_{22} Y(x, t)\right],
\end{array} \quad x \in(0, l \pi),\right.
$$

equipping with homogeneous Neumann boundary condition, where $X(x, t)$ and $Y(x, t)$ represent the densities of prey and predator populations at time $t$ and location $x$, respectively, $d_{1}, d_{2}>0$ denote the diffusion coefficients of prey and predator, $\tau$ is the generation time of the prey species, and $r_{i}, a_{i j}(i, j=1,2)$ are positive constants.

It is obvious that if $r_{1} a_{21}-r_{2} a_{11}>0,(73)$ has a unique positive equilibrium $E^{*}=\left(X^{*}, Y^{*}\right)$ [48] where

$$
X^{*}=\frac{r_{1} a_{22}+r_{2} a_{12}}{a_{11} a_{22}+a_{12} a_{21}}, Y^{*}=\frac{r_{1} a_{21}-r_{2} a_{11}}{a_{11} a_{22}+a_{12} a_{21}} .
$$

By the tanslation $u=X-X^{*}, v=Y-Y^{*}$, (73) can be written as

$$
\begin{aligned}
& \frac{\partial u(x, t)}{\partial t}=d_{1} \Delta u(x, t)+\left(u(x, t)+X^{*}\right)\left[-a_{11} u(x, t-\tau)-a_{12} v(x, t)\right], \\
& \frac{\partial v(x, t)}{\partial t}=d_{2} \Delta v(x, t)+\left(v(x, t)+Y^{*}\right)\left[a_{21} u(x, t)-a_{22} v(x, t)\right] .
\end{aligned}
$$

The linearization of (74) at the origin is

$$
\begin{aligned}
& \left.\frac{\partial u(x, t)}{\partial t}=d_{1} \Delta u(x, t)-a_{11} X^{*} u(x, t-\tau)-a_{12} X^{*} v(x, t)\right] \\
& \frac{\partial v(x, t)}{\partial t}=d_{2} \Delta v(x, t)+a_{21} Y^{*} u(x, t)-a_{22} Y^{*} v(x, t)
\end{aligned}
$$

whose characteristic equation is

$$
\lambda^{2}+A_{n} \lambda+B_{n}+e^{-\lambda \tau}\left(C \lambda+D_{n}\right)=0
$$

with $n \in \mathbb{N}_{0}$,

$$
\begin{aligned}
& A_{n}=d_{1} \frac{n^{2}}{l^{2}}+d_{2} \frac{n^{2}}{l^{2}}+a_{22} Y^{*}, \\
& B_{n}=d_{1} \frac{n^{2}}{l^{2}}\left(a_{22} Y^{*}+d_{2} \frac{n^{2}}{l^{2}}\right)+a_{12} a_{21} X^{*} Y^{*}, \\
& C=a_{11} X^{*}, \\
& D_{n}=\left(a_{22} Y^{*}+d_{2} \frac{n^{2}}{l^{2}}\right) a_{11} X^{*} .
\end{aligned}
$$

Clearly, $\lambda=0$ is not a root of (75), which excludes the existence of Turing bifurcation. When $\tau=0$, Eq. (75) becomes the following sequence of quadratic polynomial equations

$$
\lambda^{2}+\left(A_{n}+C\right) \lambda+\left(B_{n}+D_{n}\right)=0, \quad n \in \mathbb{N}_{0},
$$


where $A_{n}+C=d_{1} \frac{n^{2}}{l^{2}}+d_{2} \frac{n^{2}}{l^{2}}+a_{22} Y^{*}+a_{11} X^{*}>0, B_{n}+D_{n}=d_{1} \frac{n^{2}}{l^{2}}\left(a_{22} Y^{*}+d_{2} \frac{n^{2}}{l^{2}}\right)+$ $a_{12} a_{21} X^{*} Y^{*}+\left(a_{22} Y^{*}+d_{2} \frac{n^{2}}{l^{2}}\right) a_{11} X^{*}>0$. We know that all roots of Eq. (76) have negative real part.

We would like to seek critical values of $\tau$ such that there exists a pair of imaginary eigenvalues. Let $\pm i \omega(\omega>0)$ be the solution of Eq. (175), then we have

$$
\left\{\begin{array}{l}
-\omega^{2}+B_{n}=-C \omega \sin \omega \tau-D_{n} \cos \omega \tau, \\
A_{n} \omega=-C \omega \cos \omega \tau+D_{n} \sin \omega \tau,
\end{array}\right.
$$

which is solved by

$$
\begin{aligned}
& \sin \omega \tau=\frac{A_{n} \omega D_{n}-\left(B_{n}-\omega^{2}\right) C \omega}{(C \omega)^{2}+D_{n}^{2}}=S_{n}(\omega), \\
& \cos \omega \tau=-\frac{A_{n} C \omega^{2}+\left(B_{n}-\omega^{2}\right) D_{n}}{D_{n}^{2}+(C \omega)^{2}}=C_{n}(\omega) .
\end{aligned}
$$

Then, we obtian

$$
G(\omega)=\omega^{4}+\left(A_{n}^{2}-2 B_{n}-C^{2}\right) \omega^{2}+B_{n}^{2}-D_{n}^{2}=0
$$

Suppose that

$$
\left(H_{5}\right) A_{n}^{2}-2 B_{n}-C^{2}<0, B_{n}^{2}-D_{n}^{2}>0, \text { and }\left(A_{n}^{2}-2 B_{n}-C^{2}\right)^{2}-4\left(B_{n}^{2}-D_{n}^{2}\right)>0
$$

holds, Eq. (77) has two positive roots given by

$$
\begin{gathered}
\omega_{n}^{ \pm}=\sqrt{\frac{-\left(A_{n}^{2}-2 B_{n}-C^{2}\right) \pm \sqrt{\left(A_{n}^{2}-2 B_{n}-C^{2}\right)^{2}-4\left(B_{n}^{2}-D_{n}^{2}\right)}}{2} .} \\
\tau_{n}^{j \pm}= \begin{cases}\frac{\arccos C_{n}(\omega)+2 j \pi}{\omega_{n}^{ \pm}}, & \text {if } \mathrm{S}_{\mathrm{n}}\left(\omega_{\mathrm{n}}^{ \pm}\right)>0, \\
\frac{2 \pi-\arccos C_{n}(\omega)+2 j \pi}{\omega_{n}^{ \pm}}, & \text {if } \mathrm{S}_{\mathrm{n}}\left(\omega_{\mathrm{n}}^{ \pm}\right)<0 .\end{cases}
\end{gathered}
$$

By calculation and the results in [48], we can verify the transversality condition

$$
\left.\operatorname{SignRe} \frac{d \lambda}{d \tau}\right|_{\lambda=i \omega_{n}^{+}}>0
$$

and

$$
\left.\operatorname{SignRe} \frac{d \lambda}{d \tau}\right|_{\lambda=i \omega_{n}^{-}}<0
$$

Theorem 3. Suppose that $\left(H_{5}\right)$ holds, system (73) undergoes a Hopf bifurcation at the origin when $\tau=\tau_{n}^{j-}$ or $\tau_{n}^{j+}$.

We fix the other parameters, and choose $r_{1}$ and $\tau$ as double Hopf bifurcation parameters.

Theorem 4. Suppose that $\left(H_{5}\right)$ holds, and there exists $r_{1}^{*}$ such that $\tau_{n_{1}}^{j_{1}-}=\tau_{n_{2}}^{j_{2}+}$. Then system (73) undergoes a double Hopf bifurcation at the origin when $r_{1}=r_{1}^{*}, \tau=\tau_{n_{1}}^{j_{1}-}=$ $\tau_{n_{2}}^{j_{2}+} \triangleq \tau^{*}$. 


\subsection{Normal Form of Double Hopf Bifurcation}

Let $u(x, t)=X(x, \tau t)-X^{*}, v(x, t)=Y(x, \tau t)-Y^{*}, \tau=\tau^{*}+\alpha_{1}, r_{1}=r_{1}^{*}+\alpha_{2}$, then (73) can be written as

$$
\begin{aligned}
& \frac{\partial u(x, t)}{\partial t}=\tau^{*} d_{1} \Delta u(x, t)+\tau^{*}\left(u(x, t)+X^{*}\right)\left[-a_{11} u(x, t-1)-a_{12} v(x, t)\right], \\
& \frac{\partial v(x, t)}{\partial t}=\tau^{*} d_{2} \Delta v(x, t)+\tau^{*}\left(v(x, t)+Y^{*}\right)\left[a_{21} u(x, t)-a_{22} v(x, t)\right] .
\end{aligned}
$$

Denote $U(t)=\left(u(x, t), v(x, t)^{T}\right.$, then we have

$$
\frac{\mathrm{d} U}{\mathrm{~d} t}=D\left(\tau^{*}+\alpha_{1}, r_{1}^{*}+\alpha_{2}\right) \Delta U(t)+L\left(\tau^{*}+\alpha_{1}, r_{1}^{*}+\alpha_{2}\right) U^{t}+F\left(\tau^{*}+\alpha_{1}, r_{1}^{*}+\alpha_{2}, U^{t}\right) .
$$

Here

$$
\begin{aligned}
& D\left(\tau^{*}+\alpha_{1}, r_{1}^{*}+\alpha_{2}\right)=\left(\tau^{*}+\alpha_{1}\right)\left(\begin{array}{cc}
d_{1} & 0 \\
0 & d_{2}
\end{array}\right)=D_{0}+\alpha_{1} D_{1}^{(1,0)}+\alpha_{2} D_{1}^{(0,1)}, \\
& L\left(\tau^{*}+\alpha_{1}, r_{1}^{*}+\alpha_{2}\right) U^{t}=\left(L_{0}+\alpha_{1} L_{1}^{(1,0)}+\alpha_{2} L_{1}^{(0,1)}\right) U^{t}, \\
& F\left(\omega^{*}+\alpha_{1}, r_{1}^{*}+\alpha_{2}, U^{t}\right)=\left(\tau^{*}+\alpha_{1}\right)\left(\begin{array}{c}
-a_{11} U_{1}^{t}(-1) U_{1}^{t}(0)-a_{12} U_{1}^{t}(0) U_{2}^{t}(0) \\
a_{21} U_{1}^{t}(0) U_{2}^{t}(0)-a_{22} U_{2}^{t 2}
\end{array}\right),
\end{aligned}
$$

with

$$
\begin{gathered}
D_{0}=\tau^{*}\left(\begin{array}{ll}
d_{1} & 0 \\
0 & d_{2}
\end{array}\right), D_{1}^{(1,0)}=\left(\begin{array}{ll}
d_{1} & 0 \\
0 & d_{2}
\end{array}\right), D_{1}^{(0,1)}=0 \\
L_{0} U^{t}=\tau^{*}\left(A U^{t}(0)+G_{1} U^{t}(-1)\right), L_{1}^{(1,0)} U^{t}=A U^{t}(0)+G_{1} U^{t}(-1), \\
L_{1}^{(0,1)} U^{t}=\tau^{*} \frac{1}{a_{11} a_{22}+a_{12} a_{21}}\left[\left(\begin{array}{rr}
0 & -a_{12} a_{22} \\
a_{21} a_{21} & -a_{22} a_{21}
\end{array}\right) U^{t}(0)+\left(\begin{array}{cc}
-a_{11} a_{22} & 0 \\
0 & 0
\end{array}\right) U^{t}(-1)\right],
\end{gathered}
$$

where

$$
A=\left(\begin{array}{cc}
0 & -a_{12} X_{r_{1}^{*}}^{*} \\
a_{21} Y_{r_{1}^{*}}^{*} & -a_{22} Y_{r_{1}^{*}}^{*}
\end{array}\right), G_{1}=\left(\begin{array}{cc}
-a_{11} X_{r_{1}^{*}}^{*} & 0 \\
0 & 0
\end{array}\right),
$$

and $X_{r_{1}^{*}}^{*}=\frac{r_{1}^{*} a_{22}+r_{2} a_{12}}{a_{11} a_{22}+a_{12} a_{21}}, Y_{r_{1}^{*}}^{*}=\frac{r_{1}^{*} a_{21}-r_{2} a_{11}}{a_{11} a_{22}+a_{12} a_{21}}$.

Eq. (79) can be rewritten as

$$
\frac{\mathrm{d} U}{\mathrm{~d} t}=D_{0} \Delta U(t)+L_{0} U^{t}+\widetilde{F}\left(\alpha, U^{t}\right)
$$

where

$$
\widetilde{F}\left(\alpha, U^{t}\right)=\left(\alpha_{1} D_{1}^{(1,0)}+\alpha_{2} D_{1}^{(0,1)}\right) \Delta U+\left(\alpha_{1} L_{1}^{(1,0)}+\alpha_{2} L_{1}^{(0,1)}\right) U^{t}+F\left(\tau^{*}+\alpha_{1}, r_{1}^{*}+\alpha_{2}, U^{t}\right)
$$


Consider the linearized system of (800)

$$
\frac{\mathrm{d} U}{\mathrm{~d} t}=D_{0} \Delta U(t)+L_{0} U^{t}
$$

From the previous discussion, we know that system (81) has pure imaginary eigenvalues $\pm i \omega_{n_{2}}^{+} \tau^{*}, \pm i \omega_{n_{1}}^{-} \tau^{*}$ at the double Hopf bifurcation point and the other eigenvalues with nonzero real part. After a few calculations, we have that the bases of $P$ and $P^{*}$, respectively, are $\Phi(\theta)=\left(\phi_{1}(\theta), \bar{\phi}_{1}(\theta), \phi_{3}(\theta), \bar{\phi}_{3}(\theta)\right)$, and $\Psi(s)=\left(\psi_{1}(s), \bar{\psi}_{1}(s), \psi_{3}(s), \bar{\psi}_{3}(s)\right)^{T}$, with

$$
\begin{gathered}
\phi_{1}(\theta)=\left(1, p_{12}\right)^{T} e^{i \omega_{n_{1}}^{-} \tau^{*} \theta}, \quad \phi_{3}(\theta)=\left(1, p_{32}\right)^{T} e^{i \omega_{n_{2}}^{+} \tau^{*} \theta}, \\
\psi_{1}^{*}(s)=D_{1}\left(1, q_{12}\right) e^{-i \omega_{n_{1}}^{-} \tau^{*} s}, \quad \psi_{3}^{*}(s)=D_{2}\left(1, q_{32}\right) e^{-i \omega_{n_{2}}^{+} \tau^{*} s},
\end{gathered}
$$

where

$$
\begin{gathered}
p_{12}=\frac{-a_{11} X_{r_{1}}^{*} e^{-i \omega_{n_{1}}^{-} \tau^{*}}-i \omega_{n_{1}}^{-}-d_{1} \frac{n^{2}}{l^{2}}}{a_{12} X_{r_{1}^{*}}^{*}}, p_{32}=\frac{-a_{11} X_{r_{1}}^{*} e^{-i \omega_{n_{2}}^{+} \tau^{*}}-i \omega_{n_{2}}^{+}-d_{1} \frac{n^{2}}{l^{2}}}{a_{12} X_{r_{1}^{*}}^{*}}, \\
q_{12}=-\frac{-a_{11} X_{r_{1}}^{*} e^{-i \omega_{n_{1}}^{-} \tau^{*}}-i \omega_{n_{1}}^{-}-d_{1} \frac{n^{2}}{l^{2}}}{a_{21} Y_{r_{1}^{*}}^{*}}, q_{32}=-\frac{-a_{11} X_{r_{1}}^{*} e^{-i \omega_{n_{2}}^{+} \tau^{*}}-i \omega_{n_{2}}^{+}-d_{1} \frac{n^{2}}{l^{2}}}{a_{21} Y_{r_{1}^{*}}^{*}}, \\
D_{1}=\frac{1}{1+p_{12} q_{12}-a_{11} X_{r_{1}^{*}}^{*} \tau^{*} e^{-i \omega_{n_{1}}^{-} \tau^{*}}}, D_{2}=\frac{1}{1+p_{32} q_{32}-a_{11} X_{r_{1}^{*}}^{*} \tau^{*} e^{-i \omega_{n_{2}}^{+} \tau^{*}}} .
\end{gathered}
$$

Consider the Taylor expansion

$$
\widetilde{F}\left(\alpha, U^{t}\right)=\frac{1}{2 !} \widetilde{F}_{2}\left(\alpha, U^{t}\right)+\frac{1}{3 !} \widetilde{F}_{3}\left(\alpha, U^{t}\right),
$$

where $\widetilde{F}_{2}\left(\alpha, U^{t}\right)=2 \alpha_{1} D_{1}^{(1,0)} \Delta U+2 \alpha_{2} D_{1}^{(0,1)} \Delta U+\left(2 \alpha_{1} L_{1}^{(1,0)}+2 \alpha_{2} L_{1}^{(0,1)}\right) U^{t}+2 F\left(\omega^{*}+\alpha_{1}, d_{2}^{*}+\right.$ $\left.\alpha_{2}, U^{t}\right)$, and $\frac{1}{3 !} \widetilde{F}_{3}\left(\alpha, U^{t}\right)=0$.

By a few calculations, we have

$$
\begin{aligned}
& F_{2000}=2 \tau^{*}\left(\begin{array}{c}
-a_{11} e^{-i \omega_{n_{1}}^{-} \tau^{*}}-a_{12} p_{12} \\
a_{21} p_{12}-a_{22} p_{12}^{2}
\end{array}\right), \\
& F_{1100}=2 \tau^{*}\left(\begin{array}{c}
-a_{11}\left(e^{i \omega_{n}^{-} \tau^{*}}+e^{-i \omega_{n_{1}}^{-} \tau^{*}}\right)-a_{12}\left(p_{12}+\bar{p}_{12}\right) \\
a_{21}\left(p_{12}+\bar{p}_{12}\right)-a_{22} 2 p_{12} \bar{p}_{12}
\end{array}\right), \\
& F_{1010}=2 \tau^{*}\left(\begin{array}{c}
-a_{11}\left(e^{-i \omega_{n_{2}}^{+} \tau^{*}}+e^{-i \omega_{n_{1}}^{-} \tau^{*}}\right)-a_{12}\left(p_{12}+p_{32}\right) \\
a_{21}\left(p_{12}+p_{32}\right)-a_{22} 2 p_{12} p_{32} \\
F_{1001}=2 \tau^{*}\left(\begin{array}{c}
-a_{11}\left(e^{i \omega_{n_{2}}^{+} \tau^{*}}+e^{-i \omega_{n_{1}}^{-} \tau^{*}}\right)-a_{12}\left(p_{12}+\bar{p}_{32}\right) \\
a_{21}\left(p_{12}+\bar{p}_{32}\right)-a_{22} 2 p_{12} \bar{p}_{32}
\end{array}\right),
\end{array}\right),
\end{aligned}
$$




$$
\begin{aligned}
& F_{0200}=2 \tau^{*}\left(\begin{array}{c}
-a_{11} e^{i \omega_{n_{1}}^{-} \tau^{*}}-a_{12} \bar{p}_{12} \\
a_{21} \bar{p}_{12}-a_{22} \bar{p}_{12}^{2}
\end{array}\right) \\
& F_{0110}=2 \tau^{*}\left(\begin{array}{c}
-a_{11}\left(e^{-i \omega_{n_{2}}^{+} \tau^{*}}+e^{i \omega_{n_{1}}^{-} \tau^{*}}\right)-a_{12}\left(\bar{p}_{12}+p_{32}\right) \\
a_{21}\left(\bar{p}_{12}+p_{32}\right)-a_{22} 2 \bar{p}_{12} p_{32}
\end{array}\right) \\
& F_{0101}=2 \tau^{*}\left(\begin{array}{c}
-a_{11}\left(e^{i \omega_{n_{2}}^{+} \tau^{*}}+e^{i \omega_{n_{1}}^{-} \tau^{*}}\right)-a_{12}\left(\bar{p}_{12}+\bar{p}_{32}\right) \\
a_{21}\left(\bar{p}_{12}+\bar{p}_{32}\right)-a_{22} 2 \bar{p}_{12} \bar{p}_{32}
\end{array}\right) \\
& F_{0020}=2 \tau^{*}\left(\begin{array}{c}
-a_{11} e^{-i \omega_{n_{2}}^{+} \tau^{*}}-a_{12} p_{32} \\
a_{21} p_{32}-a_{22} p_{32}^{2}
\end{array}\right), \\
& F_{0011}=2 \tau^{*}\left(\begin{array}{c}
-a_{11}\left(e^{i \omega_{n_{2}}^{+} \tau^{*}}+e^{-i \omega_{n_{2}}^{+} \tau^{*}}\right)-a_{12}\left(p_{32}+\bar{p}_{32}\right) \\
a_{21}\left(p_{32}+\bar{p}_{32}\right)-a_{22} 2 p_{32} \bar{p}_{32} \\
-a_{11} e^{i \omega_{n_{2}}^{+} \tau^{*}}-a_{12} \bar{p}_{32} \\
a_{21} \bar{p}_{32}-a_{22} \bar{p}_{32}^{2}
\end{array}\right)
\end{aligned}
$$

By Remark 2 in Section 3, establish the linear operators

$$
\begin{aligned}
& S_{y z_{1}}(y)=F_{y(0) z_{1}} y(0)+F_{y(-1) z_{1}} y(-1), \\
& S_{y z_{2}}(y)=F_{y(0) z_{2}} y(0)+F_{y(-1) z_{2}} y(-1), \\
& S_{y z_{3}}(y)=F_{y(0) z_{3}} y(0)+F_{y(-1) z_{3}} y(-1), \\
& S_{y z_{4}}(y)=F_{y(0) z_{4}} y(0)+F_{y(-1) z_{4}} y(-1),
\end{aligned}
$$

where

$$
\begin{aligned}
& F_{y(0) z_{1}}=2 \tau^{*}\left(\begin{array}{cc}
-a_{11} e^{-i \omega_{n_{1}}^{-} \tau^{*}}-a_{12} p_{12} & -a_{12} \\
a_{21} p_{12} & a_{21}-2 a_{22} p_{12}
\end{array}\right), F_{y(-1) z_{1}}=2 \tau^{*}\left(\begin{array}{cc}
-a_{11} e^{i \omega_{n_{1}}^{-} \tau^{*}}-a_{12} \bar{p}_{12} & -a_{12} \\
a_{21} \bar{p}_{12} & a_{21}-2 a_{22} \bar{p}_{12}
\end{array}\right), F_{y(-1) z_{2}}=2 \tau^{*}\left(\begin{array}{cc}
-a_{11} & 0 \\
0 & 0
\end{array}\right), \\
& F_{y(0) z_{2}}=2 \tau^{*}\left(\begin{array}{cc}
-a_{11} & 0 \\
0 & 0
\end{array}\right), F_{y(-1) z_{3}}=2 \tau^{*}\left(\begin{array}{cc}
-a_{11} e^{-i \omega_{n_{2}}^{+} \tau^{*}}-a_{12} p_{32} & -a_{12} \\
a_{21} p_{32} & a_{21}-2 a_{22} p_{32}
\end{array}\right) \\
& F_{y(0) z_{3}}=2 \tau^{*}\left(\begin{array}{cc}
-a_{11} e^{i \omega_{n_{2}}^{+} \tau^{*}-a_{12} \bar{p}_{32}} & -a_{12} \\
a_{21} \bar{p}_{32} & a_{21}-2 a_{22} \bar{p}_{32}
\end{array}\right), F_{y(-1) z_{4}}=2 \tau^{*}\left(\begin{array}{cc}
-a_{11} & 0 \\
0 & 0
\end{array}\right),
\end{aligned}
$$

Following the steps in Remark 3, we can get all the coefficients in (153), and then obtain the normal forms up to the third order. 


\subsection{Simulations}

In this section, we fix $r_{2}=1, a_{11}=1, a_{12}=1.2, a_{21}=2.8, a_{22}=1, d_{1}=0.1, d_{2}=0.2$, $l=3$, and take $r_{1}$ and $\tau$ as bifurcation parameters. We can find that when $0.316<r_{1}<1.47$, $\left(H_{5}\right)$ holds for all $n=0$, and when $0.316<r_{1}<1.19,\left(H_{5}\right)$ holds for all $n=1$. By (78), we can draw the curves of Hopf bifurcation values when $r_{1}$ varies, which is shown in Figure 5(a). When $r_{1}=0.6739271475, \tau_{0}^{0-}$ intersects $\tau_{0}^{1+}$, and we denote the double Hopf bifurcation point HH. For HH, we have $\omega_{0}^{+}=0.77444, \omega_{0}^{-}=0.362170$, and $\tau_{0}^{0-}=\tau_{0}^{1+}=10.4238045$. We get that $B_{11}=0.17069+0.12592 i, B_{21}=1.97884+2.26811 i, B_{13}=-0.11732+0.09868 i$, $B_{23}=-3.48553+1.52722 i, B_{2100}=-0.58923-0.57368 i, B_{1011}=-7.57432+11.46887 i$, $B_{0021}=3.603569-7.55242 i, B_{1110}=0.374678+2.89123 i, \epsilon_{1}=-1, \epsilon_{2}=1, b_{0}=2.10189$, $c_{0}=-0.63587, d_{0}=-1$, and $d_{0}-b_{0} c_{0}=0.336547$. It means that the unfolding system is of type VIa, and the bifurcation set is shown in Figure 5(b) in which the phase portraits in D1-D8 have been shown in Figure 2. System (73) has a quasi-periodic solution on a 2-torus and a quasi-periodic solution on a 3-torus, which will be eliminated by a saddle connection orbit. According to the "Ruelle-Takens-Newhouse" scenario to chaos [4, 16, 47], a vanishing 3-torus might accompany strange attractors, and lead a system into chaos. Thus, we know that near point $\mathrm{HH}$, there may exist a strange attractor, which will be shown numerically in Figure 6.

In Figure 6, we show the existence of quasi-periodic solutions with multiple frequencies, and show the results through the Poincaré map on a Poincaré section. Since all periodic or quasi-periodic solutions are spatially homogeneous, we choose the solution curve of $(X(0, t), Y(0, t))$, i.e., $x=0$. Moreover, because the periodic solutions oscillate in an infinite dimensional phase space [53], we give simulations near the double Hopf bifurcation point on the Poincaré section $X(0, t-\tau)=X^{*}$. We can see that the system exhibits rich dynamical behavior near the bifurcation point HH in Figure 6. System (73) has a quasi-periodic solution on a 2-torus which becomes a quasi-periodic solution on a 3-torus and then vanishes through a heteroclinic orbit, which is shown in Figure 6 (a) and (b) respectively. After the vanishing of 3-torus, system (73) has a strange attractor, and exhibits chaotic behavior, which is shown in 6 (c). 


\section{CONCLUDING REMARKS}

In this paper, we have extended the center manifold reduction and normal form method to analyze the dynamical behavior near the double Hopf bifurcation point in a delayed reaction-diffusion system. The method appears to be quite complicated as proceeded in this paper, but it is still an explicit algorithm and is not difficult to be implanted into a computer program. What we should care more about in this method is to calculate the double Hopf point and all the rest part can be exactly obtained by following the procedure we have given.

After normal form derivation with respect to a delayed reaction-diffusion system, the dynamics near the bifurcation point, like the case in ordinary differential equations, is also governed by twelve distinct kinds of unfolding systems, and the bifurcation set about ordinary differential equations for each of the twelve types of unfoldings still applies to our model. To show that our method is a powerful method for analyzing local behavior around a double Hopf bifurcation point, we give two examples: in the stage-structured epidemic model, we show that two spatially inhomogeneous periodic oscillations coexist near the singular point; in the predator-prey system, we show the existence of quasi-periodic solution

on a 2-torus, quasi-periodic solution on a 3-torus, and strange attractor appears near the bifurcation point.

\section{ACKNOWLEDGMENTS}

The authors are grateful to the handling editor and anonymous referees for their careful reading of the manuscript and valuable comments, which improve the exposition of the paper very much. This research is supported by National Natural Science Foundation of China (11701120, 11771109) and Shaanxi Provincial Education Department grant (18JK0123).

[1] An, Q., Jiang, W: Spatiotemporal attractors generated by the Turing-Hopf bifurcation in a time-delayed reaction-diffusion system. Disctete Cont. Dyn-B doi: 10.3934/dcdsb.2018183 (2018)

[2] Andronov, A.A.: Application of Poincaré theorem on bifurcation points and change in stability to simple auto-oscillatory systems. C. R. Acad. Sci. Paris 189, 559-561 (1929) 
[3] Bajaj, A.K., Sethna, P.R.: Bifurcations in three-dimensional motions of articulated tubes. I Linear systems and symmetry. II - Nonlinear analysis. J. Appl. Mech. 49, 606-618 (1982)

[4] Battelino, P.M., Grebogi, C., Ott, E., Yorke, J.A.: Chaotic attractors on a 3-torus, and torus break-up. Physica D 39, 299-314 (1989)

[5] Baurmann, M., Gross, T., Feudel, U.: Instabilities in spatially extended predator-prey systems: spatio-temporal patterns in the neighborhood of Turing-Hopf bifurcations. J. Theor. Bio. 245, 220-229 (2007)

[6] Belair, J., Campbell, S.A., Driessche, P.V.D.: Frustration, stability, and delay-induced oscillations in a neural network model. SIAM. J. Appl. Math. 56, 245-255 (1996)

[7] Bi, P., Ruan, S.: Bifurcations in delay differential equations and applications to tumor and immune system interaction models. SIAM J. Appl. Dyn. Syst. 12, 1847-1888 (2013)

[8] Buono, P.L., Bélair, J.: Restrictions and unfolding of double Hopf bifurcation in functional differential equations. J. Differ. Equ. 189, 234-266 (2003)

[9] Campell, S.A., Bélair, J.: Analytical and symbolically-assisted investigation of Hopf bifurcations in delay-differential equations. Can. Appl. Math. Q. 3, 137-154 (1995)

[10] Campell, S.A., Bélair, J., Ohira, T., Milton, J.: Limit cycles, tori, and complex dynamics in a second-order differential equations with delayed negative feedback. J. Dyn. Differ. Equ. 7, 213-236 (1995)

[11] Campell, S.A., LeBlanc, V.G.: Resonant Hopf-Hopf interaction in delay differential equations. J. Dyn. Differ. Equ. 10, 327-346 (1998)

[12] Chen, S., Shi, J., Wei, J.: Global stability and Hopf bifurcation in a delayed diffusive LeslieGower predator-prey system, Int. J. Bifurcat. Chaos 22, 331-517 (2012)

[13] Chen, S., Yu, J.: Stability and bifurcations in a nonlocal delayed reaction-diffusion population model. J. Differ. Equations 260, 218-240 (2016)

[14] De Wit, A., Dewel, G., Borckmans, P.: Chaotic Turing-Hopf mixed mode. Phys. Rev. E 48, R4191-R4194 (1993)

[15] Du, Y., Guo, Y., Xiao, P.: Freely-moving delay induces periodic oscillations in a structured SEIR model. Int. J. Bifurcat. Chaos 27, 1750122 (2017)

[16] Eckmann, J.P.: Roads to turbulence in dissipative dynamical systems. Rev. Modern Phys. 53, 643-654 (1981)

[17] Elphick, C., Tiraopegui, E., Brachet, M.E., Coullet, P., Iooss, G.: A simple global character- 
ization for normal forms of singular vector fields. Physica D 29, 95-127 (1987)

[18] Faria, T.: Normal forms and Hopf bifurcation for partial differential equations with delays. Trans. Amer. Math. Soc. 352, 2217-2238 (2000)

[19] Faria, T., Stability and bifurcation for a delayed predator-prey model and the effect of diffusion. J. Math. Anal. Appl. 254, 433-463 (2001)

[20] Faria, T., Huang, W.: Stability of periodic solutions arising from Hopf bifurcation for a reaction-diffusion equation with time delay. Fields Inst. Comm. 31, 125-141 (2002)

[21] Faria, T., Magalhães, L.T.: Normal forms for retarded functional differential equations with parameters and applications to Hopf bifurcation. J. Differ. Equations 122, 181-200 (1995)

[22] Faria, T., Magalhães, L.T.: Normal form for retarded functional differential equations and applications to Bogdanov-Takens singularity. J. Differ. Equations 122, 201-224 (1995)

[23] Gils, S.A.V., Krupa, M., Langford, W.F.: Hopf bifurcation with non-semisimple 1:1 resonance. Nonlinearity 3, 825-850 (1990)

[24] Govaerts, W., Guckenheimer, J., Khibnik, A.: Defining functions for multiple Hopf bifurcations. SIAM J. Numer. Anal. 34, 1269-1288 (1997)

[25] Guckenheimer, J., Holmes, P.: Nonlinear Oscillations, Dynamical Systems, and Bifurcations of Vector Fields. Springer, New York (1983)

[26] Guo, S.: Stability and bifurcation in a reaction-diffusion model with nonlocal delay effect. J. Differ. Equations 259, 1409-1448 (2015)

[27] Guo, S., Ma, L.: Stability and bifurcation in a delayed reaction-diffusion equation with Dirichlet boundary condition. J. Nonl. Sci. 26, 545-580 (2016)

[28] Hale, J.K., Kocak, H.: Dynamics and Bifurcations. Springer, New York (1991)

[29] Hale, J.K., Lunel, S.M.V.: Introduction to Functional Differential Equations. Springer, New York (1993)

[30] Hassard, B.D., Kazarinoff, N.D., Wan, Y.H.: Theory and Applications of Hopf Bifurcation. Cambridge Univ. Press, New York (1981)

[31] Hethcote, H.W., Lewis, M.A., Driessche, P.V.D.: An epidemiological model with a delay and a nonlinear incidence rate. J. Math. Biol. 27, 49-64 (1989)

[32] Hopf, E.: Abzweigung einer periodischen lösung eines Differential Systems. Berichen Math. Phys. Kl. Säch. Akad. Wiss. Leipzig 94, 1-22 (1942)

[33] Hsu, S.B., Huang, T.W.: Global stability for a class of predator-prey systems. SIAM J. Appl. 
Math. 55, 763-783 (1995)

[34] Ji, J., Li, X., Luo, Z.: Two-to-one resonant Hopf bifurcations in a quadratically nonlinear oscillator involving time delay. Int. J. Bifurcat. Chaos 22, 1250060 (2012)

[35] Kielhöfer, H.: Bifurcation Theory: An Introduction with Applications to Partial Differential Equations. Springer, New York (2011)

[36] Kuznetsov, Y.A.: Elements of Applied Bifurcation Theory. Springer, New York (2011)

[37] Lewis, G.M., Nagata, W.: Double Hopf bifurcations in the differentially heated rotating annulus. SIAM J. Appl. Math. 63, 1029-1055 (2003)

[38] Lin, X., So, J.W.H., Wu, J.: Centre manifolds for partial differential equations with delays. P. Roy. Soc. Edinb. A 122, 237-254 (1992)

[39] Luongo, A., Paolone, A.: Perturbation methods for bifurcation analysis from multiple nonresonant complex eigenvalues. Nonlinear Dynam. 14, 193-210 (1997)

[40] Ma, S., Lu, Q., Feng, Z.: Double Hopf bifurcation for van der Pol-Duffing oscillator with parametric delay feedback control. J. Math. Anal. Appl. 338, 993-1007 (2008)

[41] Meixner, M., De Wit, A., Bose, S., Schöll, E.: Generic spatiotemporal dynamics near codimension-two Turing-Hopf bifurcations. Phys. Rev. E 55, 6690-6697 (1997)

[42] Poincaré, H.: Les Méthodes Nouvelles de la Mécanique Céleste. Cauthier-Villars, Paris (1892)

[43] Reddy, D.V.R., Sen, A., Johnston, G.L.: Time delay effects on coupled limit cycle oscillators at Hopf bifurcation. Physica D 129, 15-34 (1999)

[44] Revel, G., Alonso, D.M., Moiola, J.L.: Interactions between oscillatory modes near a 2:3 resonant Hopf-Hopf bifurcation. Chaos 20, 113-129 (2010)

[45] Revel, G., Alonso, D.M., Moiola, J.L.: Numerical semi-global analysis of a 1:2 resonant HopfHopf bifurcation. Physica D 247, 40-53 (2013)

[46] Ruan, S., Xiao, D.: Global analysis in a predator-prey system with nonmonotonic functional response. SIAM J. Appl. Math. 61, 1445-1472 (2000)

[47] Ruelle, D., Takens, F.: On the nature of turbulence. Comm. Math. Phys. 20, 167-192 (1971)

[48] Song, Y., Wei, J.: Local Hopf bifurcation and global periodic solutions in a delayed predatorprey system. J. Math. Anal. Appl. 301, 1-21 (2005)

[49] Song, Y., Zhang, T., Peng, Y.: Turing-Hopf bifurcation in the reaction-diffusion equations and its applications. Commun. Nonlinear Sci. Numer. Simulat. 33, 229-258 (2016)

[50] Steen, P.H., Davis, S.H.: Quasiperiodic bifurcation in nonlinearly-coupled oscillators near a 
point of strong resonance. SIAM J. Appl. Math. 42, 1345-1368 (1982)

[51] Su, Y., Wei, J., Shi, J.: Hopf bifurcations in a reaction-diffusion population model with delay effect. J. Differ. Equations 247, 1156-1184 (2009)

[52] Wiggins, S.: Introduction to Applied Nonlinear Dynamical Systems and Chaos. Springer, New York (2003).

[53] Wu, J.: Theory and Applications of Partial Functional-Differential Equations. Springer, New York (1996)

[54] Xiao, D.: Bifurcations of a ratio-dependent predator-prey system with constant rate harvesting. SIAM J. Appl. Math. 65, 737-753 (2005)

[55] Xiao, Y., Chen, L.: An SIS epidemic model with stage structure and a delay. Acta Math. Appl. Sin. E. 18, 607-618 (2002)

[56] Xu, X., Wei, J.: Turing-Hopf bifurcation of a class of modified Leslie-Gower model with diffusion. Disc. Continu. Dyn. Sys. B 23, 765-783 (2018)

[57] Yan, X., Li, W.: Stability of bifurcating periodic solutions in a delayed reaction-diffusion population model. Nonlinearity 23, 1413-1431 (2010)

[58] Yi, F., Wei, J., Shi, J.: Bifurcation and spatiotemporal patterns in a homogenous diffusive predator-prey system. J. Differ. Equations 246, 1944-1977 (2009)

[59] Yu, P.: Analysis on double Hopf bifurcation using computer algebra with the aid of multiple scales. Nonlinear Dynamics 27, 19-53 (2002)

[60] Yu, P., Bi, Q.: Analysis of non-linear dynamics and bifurcations of a double pendulum. J. Sound Vib. 217, 691-736 (1998)

[61] Yu, P., Yuan, Y., Xu, J.: Study of double Hopf bifurcation and chaos for oscillator with time delay feedback. Commun. Nonlinear Sci. Numer. Simul. 7, 69-91 (2002)

[62] Zhang, Y., Xu, J.: Classification and computation of non-resonant double Hopf bifurcations and solutions in delayed van der Pol-Duffing system. Int. J. Nonlinear Sci. Numer. Simul. 6, 67-74 (2005) 
a)
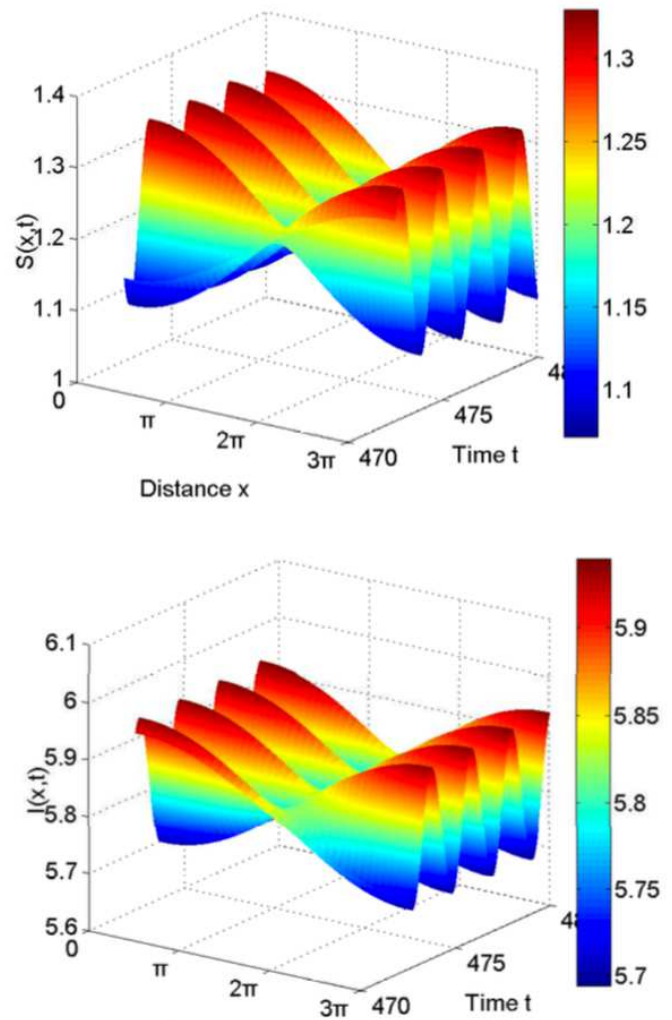

b) Distance $x$

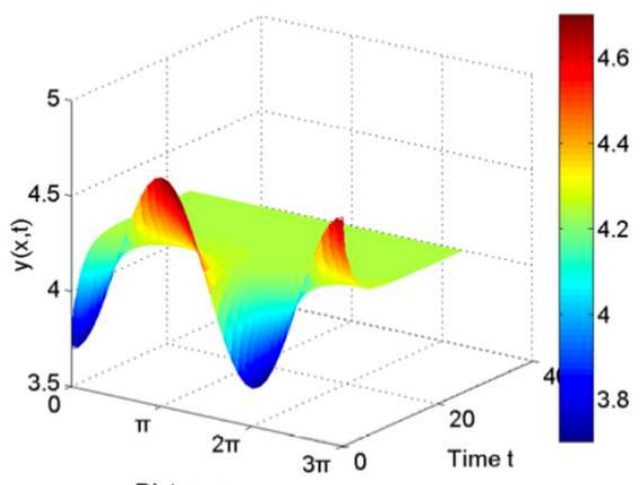

d)

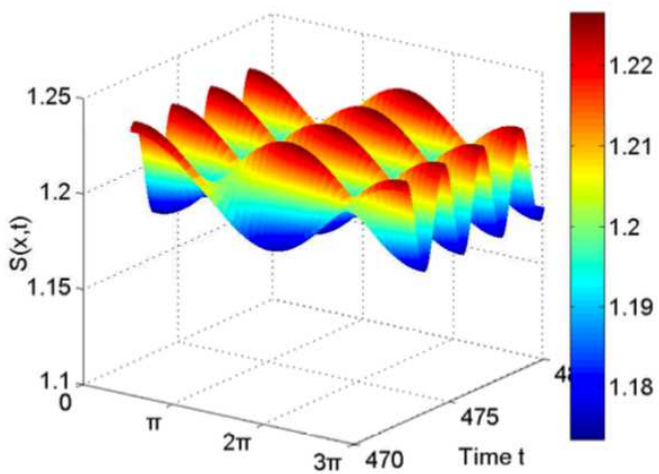

e)

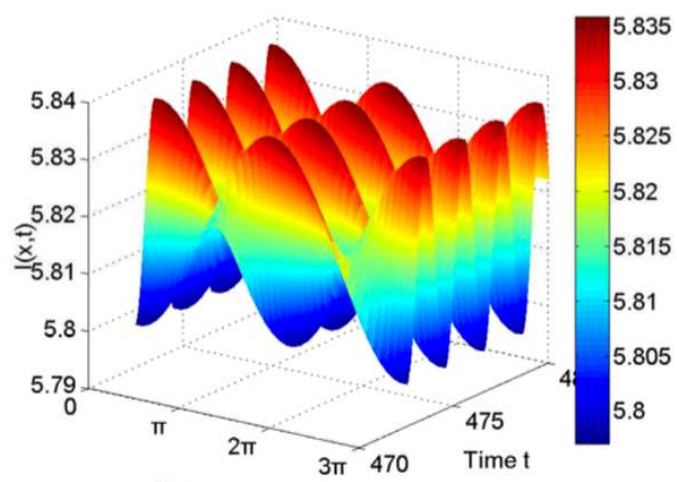

f)

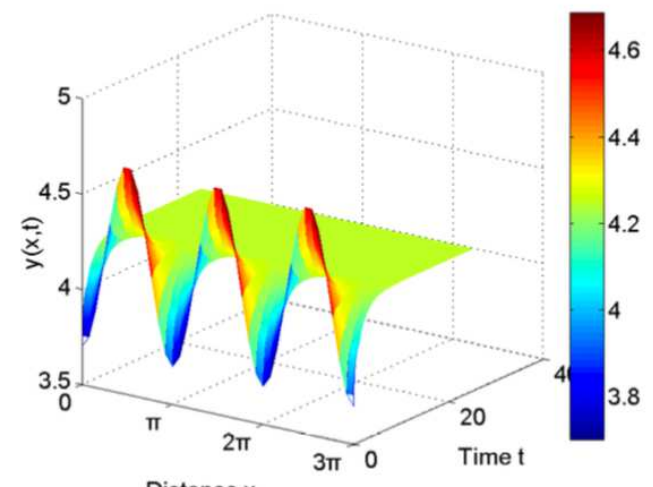

c)

Distance $x$

FIG. 4. When $d_{2}=5.23$, and $\omega=0.53$ in $D 4$, two stable nonhomogeneous periodic solutions coexist when we choose two different initial conditions. For (a-c) initial conditions are $S(x, t)=$ $1.2+0.01 \cos x, I(x, t)=5.8-0.06 \cos x, y(x, t)=4.2-0.05 \cos x, t \in[-\tau, 0]$. For (d-f) initial conditions are $S(x, t)=1.2+0.01 \cos 2 x, I(x, t)=5.8-0.06 \cos 2 x, y(x, t)=4.2-0.05 \cos 2 x$, $t \in[-\tau, 0]$. 
a)

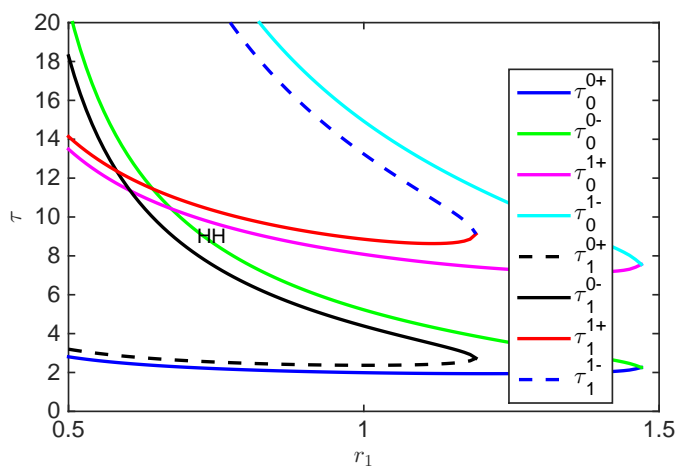

b)

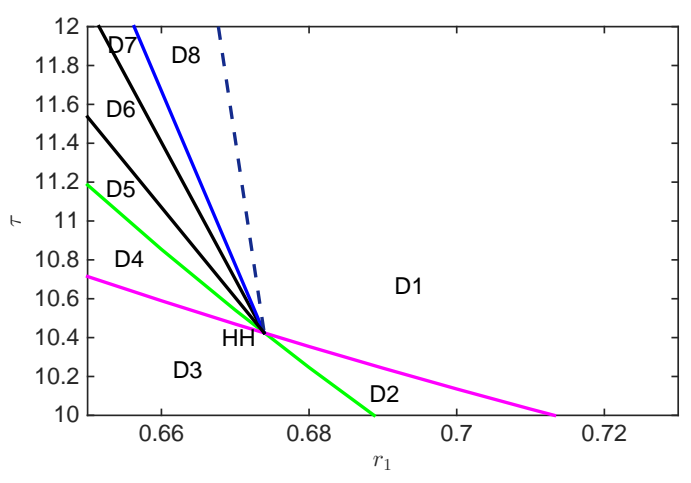

FIG. 5. When $r_{2}=1, a_{11}=1, a_{12}=1.2, a_{21}=2.8, a_{22}=1, d_{1}=0.1, d_{2}=0.2, l=3$, (a) the partial bifurcation set on the $r_{1}-\tau$ plane and (b) the complete bifurcation set around $\mathrm{HH}$ are shown.
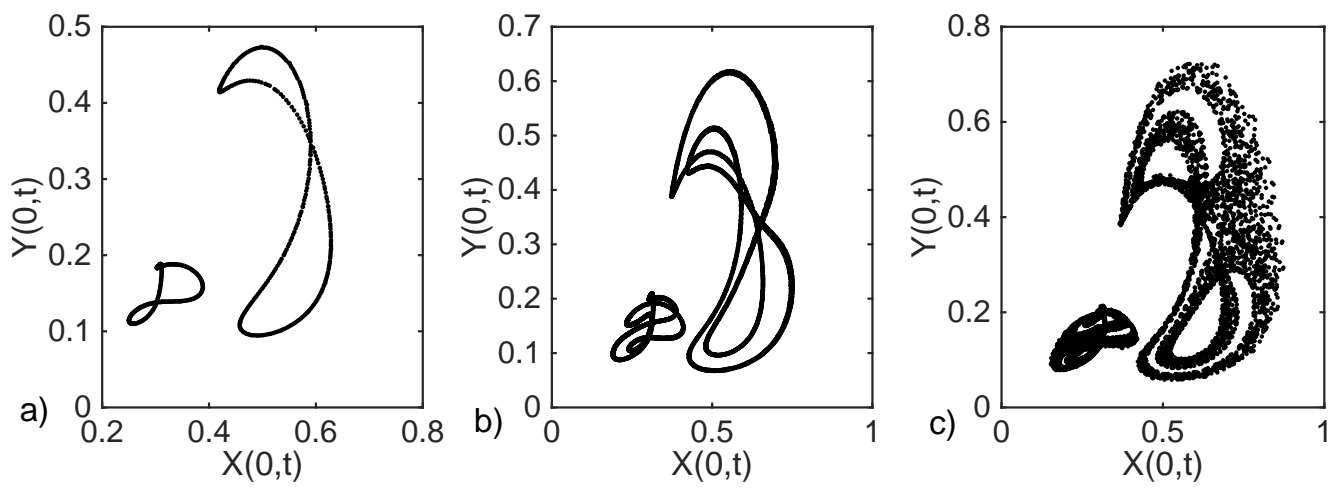

FIG. 6. (a) When $\tau=10.8, r_{1}=0.69$, there exists a stable quasi-periodic solution on a 2-torus of system (73) ; (b) when $\tau=10.8, r_{1}=0.71$, there exists a quasi-periodic solution on a 3 -torus of system (73); (c) $\tau=10.8, r_{1}=0.726$, there is a strange attractor of system (73), and the system exhibits chaotic behavior. 